\title{
Procedural rationality, asset heterogeneity and market selection
}

\author{
Guillaume COQUERET ${ }^{\mathrm{a}, *}$, Bertrand TAVIN ${ }^{\mathrm{a}}$ \\ ${ }^{a}$ EMLYON Business School, 23 Avenue Guy de Collongue, 69130 Ecully, FRANCE
}

\begin{abstract}
We extend the agent-based model of Anufriev and Bottazzi (2010) to the case with many risky assets. We show that under the procedural equilibrium, all assets with nonzero aggregate demand must have the same price returns. Heterogeneity in price returns appears when some assets face zero demand. In this case, the returns are essentially driven by the matrix of wealth-weighted products between demands and they generate a rich variety of patterns for agents' market shares. We formalize the dynamics of deterministic skeletons in our market model and consider the associated Jacobian matrix, for which we provide a closed form expression up to a matrix inversion. We then introduce the characteristic ratio of an investment strategy, which involves the average dividend yields of the risky assets and the investors' portfolio compositions. When equilibrium returns are uniform and when the number of assets is not smaller than the number of agents, the equilibrium with a single survivor can only be stable if the survivor has the maximal characteristic ratio. Moreover, we prove that this stability criterion holds as long as the noise in the system is sufficiently small. We confirm all our findings with a thorough empirical analysis of numerical simulations, with and without noise. All in all, our results form a theoretical rationale for portfolio strategies tilted towards firms that pay high dividend yields.
\end{abstract}

Keywords: Heterogeneous Agents, Multi-Asset Framework, Procedural Rationality, Market Selection, Stability Analysis, Deterministic Skeleton

JEL: G12, C62, D81

\section{Introduction}

The purpose of most mathematical models is to propose an intelligible - thus simplified - version of an observable phenomenon, for example in physics, biology, finance or economics. The construction of any model is the result of an inevitable trade-off. A simplistic model will be computationally tractable, but will often fail to capture some desired properties of the phenomenon being modeled. Complicated models are usually built to overcome this limitation, but they are, by nature much harder to handle: closed-form solutions do not exist and they can only be quantitatively characterized with the help of numerical methods, such as discretizations, simulations, approximations, etc. In the extreme case when the defining assumptions are very general, and hence ambitious, the solution cannot be described beyond its existence. Thus, it is a genuine challenge to propose a framework for which intelligible relationships can be analytically deduced from realistic, non-trivial assumptions.

In this paper, we make an attempt in this direction by generalizing the economic setting of Anufriev and Bottazzi (2010) to the case when more than one risky asset can be traded. We provide

${ }^{*}$ Corresponding author: EMLYON Business School, 23 Avenue Guy de Collongue, 69130 Ecully, FRANCE. Tel.: $+33(0) 478337845$.

Email addresses: coqueret@em-lyon.com (Guillaume COQUERET), tavin@em-lyon.com (Bertrand TAVIN) 
comprehensive insights on the links between asset returns, dividend yields, portfolio compositions and market shares when the equilibrium is of procedural type. We also determine the connection between market selection, investment strategies and asset characteristics.

The themes we discuss are strongly related to the literature on Heterogeneous Agent Models (HAMs). While most frameworks are initially intended for multiple agents and assets, the main contributions are usually obtained for a limited number of assets and/or agents. For instance, the seminal paper by Brock and Hommes (1998) includes only one risky asset and up to four agent types. ${ }^{1}$. These agent types often correspond to stylized investment strategies in which investors believe that prices either revert to their fundamental values (fundamentalists), confirm recent tendencies (trend-chasers) or oscillate in the short term (contrarians).

The purpose of the present paper is to allow for a larger palette of assets and agent demands. Some contributions in this direction include the beliefs-based CAPM of Chiarella et al. (2010) and Chiarella et al. (2013), the very complete chapter of Evstigneev et al. (2009), the empirical tests of Coqueret (2017) and the demand-driven model of Koijen and Yogo (2017).

Our study is closest to that of Anufriev and Bottazzi (2010), as we propose an extension of their economic setting. Nevertheless, while they focus on the characterization and stability of equilibria, we take another route and shed some light on market selection and deterministic skeletons. Our approach also strongly relates to that of Anufriev et al. (2012), but in contrast to their results, we will show that most often surviving agents can have inhomogeneous demands. Also, even though we do provide insights on the relationship between asset returns, it is not the primary goal of the paper.

Rather, we choose to investigate the evolution of market shares and our interest lies in their terminal values. The topic of market selection is increasingly popular, especially in evolutionary finance, and we refer to Yan (2008), Palczewski and Schenk-Hoppé (2010) and Kogan et al. (2016) for models in continuous time and to Amir et al. (2005), Hens and Schenk-Hoppé (2005), Amir et al. (2013) and Bottazzi and Dindo (2014) for models in discrete time. Earlier seminal models of market selection, e.g., Blume and Easley (1992) and Sandroni (2000), evaluate market selection based on agents' consumption choices and on the accuracy of their predictions. In these models, trajectories are exogenous to agent demands: they depend on states of nature.

In sum, our framework is that of Anufriev and Bottazzi (2010), but the main topic is market selection, as in Evstigneev et al. (2008). The main difference between the two models lies in the complement to risky assets. In Evstigneev et al. (2008), what is not invested on the market is consumed while in the present paper, the portion that is not invested in risky assets goes to the risk-less asset. As such, our model is purely financial in nature. ${ }^{2}$

\footnotetext{
${ }^{1}$ The present paper strongly relates to the literature inspired by the ideas of Brock and Hommes (1998) In continuous time, results can be found in Bhamra and Uppal (2014) and Buraschi et al. (2014) and the references therein. In discrete time, the recent work of $\mathrm{Li}$ et al. (2016) spans two asset classes and also four trading strategies. The following articles all consider at least one small dimension:

- One asset, two traders: Hommes et al. (2005)

- One asset, many traders: Brock et al. (2005), Shapiro (2009), Anufriev and Dindo (2010)

- Two traders, many assets: Chiarella et al. (2007).

${ }^{2}$ Both choices can be justified. For instance, the model of Evstigneev et al. (2008) corresponds to a setting in which the agent will need to retrieve cash-flows regularly. This is relevant for a retired individual with no labor income. If we think of an active individual, the consumption is covered by his labor income or jobseeker allowance. As a result, he invests a portion of his savings in assets directly or via a mutual fund. The endowment and potential gains will be retrieved after a long period of time, say, more than 10 years, and the individual is only concerned with pure financial performance, as in realization utility preferences (see e.g., Barberis and Xiong (2012), Ingersoll and Jin (2012) and Frydman et al. (2014)). The discussion of the relevance of either configuration lies beyond the scope
} 
Lastly, the final theme we discuss is the approximation of random systems by their deterministic skeletons. The literature provides results in this direction. In Zhu et al. (2011b), the authors investigate the relationship between the deterministic skeleton of an heterogeneous agent model and its stochastic counterpart, namely when noise is added to the dividend process. To do so they rely on results obtained in Zhu et al. (2011a). The textbook Freidlin and Wentzell (2012) details an analysis of the effect of random noise added to dynamical systems in continuous time. In Dieci et al. (2006), the authors rely on the analysis of the deterministic skeleton as well as simulations to study the evolution of the market share of different types of traders. They argue that the deterministic skeleton plays an important role in understanding the dynamics of the stochastic system. The concept of deterministic skeleton has also been used in operations research, for example in Besbes and Zeevi (2012). Finally, the deterministic approximation of a stochastic system is also addressed by Benaïm and Weibull (2003), but through the lens of game theory.

In the layout of our results, we start by considering two different cases. When aggregate demands are all non-null, we are able to characterize a condition under which an agent will survive at equilibrium: his profits are persistently higher than the wealth-weighted average profit. If agents have all the same level of leverage in risky assets (or, say, consumption rate as in Evstigneev et al. (2008)), then this strategy is to invest in the highest dividend paying asset in a proportion that is arbitrarily close to $100 \%$. The second case is when demands violate the no short-selling restriction. It can then happen that the model becomes degenerate. But when it does not, interesting patterns emerge and we illustrate them in the case of deterministic skeletons. Unfortunately, it is then much harder to determine a dominating strategy. In fact, it is highly probable that domination in this case is also determined by initial market shares.

Our contributions can be summarized as follows. We extend the model of Anufriev and Bottazzi (2010) to the case with many assets. We discuss the configurations in asset demands which imply homogeneity or heterogeneity in equilibrium price returns. We provide and discuss the corresponding equilibrium relationships between returns, demands, market shares and dividend yields. Finally, we study market selection under deterministic skeletons. When aggregate demands are non null, a criterion that differentiates strategies is able to warrant survival upon equilibrium. Furthermore, we provide a characterization of the noise in the system under which survival holds in some almost-sure sense. When some aggregate demands are null, this criterion fails and survival will be conditioned on initial market shares.

The remainder of the paper is structured as follows. In Section 2, we present the model and we obtain the dynamics of the key variables under the condition ensuring that prices remain positive. In Section 3, we characterize the PCE of our market. To do so, we identify two situations whether there exists at least an asset with zero aggregate demand or not. In Section 4 we deal with the discrete dynamics of our market model by means of the associated Jacobian matrix. Market selection and stability of fixed-points with a single survivor are solved in Section 5 where we introduce the notion of characteristic ratio associated to a strategy. In Section 6, we provide empirical illustrations of how the market dynamics behave in the two situations, with and without zero aggregate demand assets. Moreover, in the latter two sections, we clarify the conditions under which random shocks on dividends do not affect our asymptotic results. Finally, we conclude in Section 7. All proofs are gathered in the Appendices of the paper.

of this paper. Clearly, our choice is to focus on the financial market and not on the entire economy. 


\section{The model}

The economic setting is exactly the same as that of Anufriev and Bottazzi (2010), except that we consider $N$ agents and $K$ assets. We summarize the framework briefly. $W_{t, n}$ and $x_{t, n}^{(k)}$ will stand for the wealth of investor $n$ at time $t$ and for the fraction of his wealth invested in the $k^{t h}$ asset. Throughout the paper, the superscript ${ }^{(k)}$ will always refer to assets and their time- $t$ prices are written $P_{t}^{(k)}$. We assume that consumption is ignored and each agent's wealth is reinvested so that at time $t$, agent $n$ possesses $W_{t, n} x_{t, n}^{(k)} / P_{t}^{(k)}$ shares of asset $k$ and $\left(1-\sum_{k=1}^{K} x_{t, n}^{(k)}\right) W_{t, n}$ shares of the risk-less asset. We assume that, at all time, the rank of the $(K \times N)$ demands' matrix $\boldsymbol{x}_{t}$ is full, that is, equal to $\min (K, N)$. This simply means that assets or agents are not redundant. At the beginning of time $t+1$, asset $k$ generates a random positive dividend $D_{t+1}^{(k)}$ per share while the risk-less asset yields a cashflow with constant rate $r_{f}>0$.

The dividend process $D=\left\{D_{t}, t \geq 0\right\}$ is a $K$-dimensional positively valued stochastic process in discrete time. It is defined on the probability space $\left(\Omega,\left(\mathcal{F}_{t}\right)_{t \in \mathbb{N}}, \mathbb{P}\right)$, where $\Omega$ is the set of the states of nature, $\mathbb{P}$ is the true (historical) probability and $\mathcal{F}=\left\{\mathcal{F}_{t}, t \geq 0\right\}$ is the filtration generated by $D$. At time $t, \mathcal{F}_{t}$ is the $\sigma$-algebra representing the information available to the agents. Said differently, the time $t$ state of the system is $\mathcal{F}_{t}$-measurable.

The risk-less asset has a totally elastic supply and as such, its price remains unchanged. The dynamics of the wealths can then be written as:

$$
W_{t+1, n}\left(\boldsymbol{P}_{t+1}\right)=\left(1-\sum_{k=1}^{K} x_{t, n}^{(k)}\right) W_{t, n}\left(1+r_{f}\right)+W_{t, n} \sum_{k=1}^{K} x_{t, n}^{(k)} \frac{P_{t+1}^{(k)}+D_{t+1}^{(k)}}{P_{t}^{(k)}},
$$

where $\boldsymbol{P}_{t+1}$ is the vector of all risky assets' prices at time $t+1$. We underline that the above equation shows the main difference between our model and that of Evstigneev et al. (2008). In the above equation, what is not invested goes to the risk-less asset while in Evstigneev et al. (2008), it goes to consumption. We work with a constant supply of risky assets and $\boldsymbol{P}_{t+1}$ is set so as to equate aggregate supply and demand for all assets, i.e.:

$$
\sum_{n=1}^{N} W_{t+1, n}\left(\boldsymbol{P}_{t+1}\right) x_{t+1, n}^{(k)}=P_{t+1}^{(k)}, \quad k=1, \ldots, K .
$$

Now, as is noted in Anufriev and Bottazzi (2010), given the accumulation of dividends, the economy will grow continuously and it is more relevant to work with the discounted version of the model, in which the key quantities are normalized by $\left(1+r_{f}\right)$ at each period:

$$
w_{t, n}=\frac{W_{t, n}}{\left(1+r_{f}\right)^{t}}, \quad p_{t}^{(k)}=\frac{P_{t}^{(k)}}{\left(1+r_{f}\right)^{t}}, \quad e_{t}^{(k)}=\frac{D_{t}^{(k)}}{P_{t-1}^{(k)}\left(1+r_{f}\right)} .
$$

We underline that the filtration $\mathcal{F}$ can be equivalently defined as being the filtration generated by the process $e$. To ease notation, we rewrite the discounted version of system (1) and (2) in vector format:

$$
\left\{\begin{array}{rl}
\boldsymbol{p}_{t+1} & =\boldsymbol{x}_{t+1} \boldsymbol{w}_{t+1} \\
\boldsymbol{w}_{t+1} & =\operatorname{diag}\left(\mathbf{1}_{N}+\boldsymbol{x}_{t}^{\prime}\left[\operatorname{diag}\left(\boldsymbol{p}_{t}\right)^{-1} \boldsymbol{p}_{t+1}-\mathbf{1}_{K}+\boldsymbol{e}_{t+1}\right]\right) \boldsymbol{w}_{t} \\
& =\operatorname{diag}\left(\boldsymbol{w}_{t}\right)\left(\mathbf{1}_{N}+\boldsymbol{x}_{t}^{\prime}\left[\operatorname{diag}\left(\boldsymbol{p}_{t}\right)^{-1} \boldsymbol{p}_{t+1}-\mathbf{1}_{K}+\boldsymbol{e}_{t+1}\right]\right)
\end{array},\right.
$$

where $\boldsymbol{w}_{t+1}$ is the $N$-dimensional column vector of all discounted wealths and $\boldsymbol{x}_{t+1}$ is the $(K \times N)$ matrix of invested fractions. ${ }^{3}$ Moreover, $\boldsymbol{e}_{t}$ stands for the $(K \times 1)$ column vector of dividend yields

\footnotetext{
${ }^{3}$ In the sequel, we alternatively speak of investment fractions, agent demands or portfolio strategies when referring to $\boldsymbol{x}_{t}$.
} 
and $\operatorname{diag}(\cdot)$ is the operator which maps a vector into a diagonal matrix of the same dimension with diagonal values equal to the elements of the vector. As is customary, $\boldsymbol{v}^{\prime}$ and $\boldsymbol{M}^{\prime}$ denote the transpose of vector $\boldsymbol{v}$ and matrix $\boldsymbol{M}$. Finally, the curvy strict inequality $\boldsymbol{v} \succ \mathbf{0}_{N}$ denotes element-wise inequalities.

We will use the following notations throughout the paper:

- $\mathbf{1}_{N}$ is the unit column vector in $\mathbb{R}^{N}$;

- $\mathbf{0}_{N}$ is the zero column vector in $\mathbb{R}^{N}$;

- $\boldsymbol{I}_{N}$ is the $N$-dimensional identity matrix;

- $\mathbf{0}_{K, N}$ is the $(K \times N)$ matrix filled with zeros.

In addition, $\boldsymbol{\lambda}_{t}=\boldsymbol{x}_{t}^{\prime} \mathbf{1}_{K}$ will denote the aggregate risky positions of agents, and we will refer to these quantities as their leverage. The following result sums up the basic properties of the system defined in Equation (4). It generalizes Proposition 2.1 of Anufriev and Bottazzi (2010) to a multiasset framework. In essence, it shows the relationship between assets' price returns $\boldsymbol{r}_{t}$ and agents' market shares $\phi_{t}$ defined as:

$$
\boldsymbol{r}_{t+1}=\operatorname{diag}\left(\boldsymbol{p}_{t}\right)^{-1} \boldsymbol{p}_{t+1}-\mathbf{1}_{K}, \quad \boldsymbol{\phi}_{t}=\boldsymbol{w}_{t} /\left(\mathbf{1}_{N}^{\prime} \boldsymbol{w}_{t}\right) .
$$

Proposition 1. Under the assumption that the initial price vector $\boldsymbol{p}_{0}$ has only positive elements, the prices will remain positive provided that

$$
\left(\operatorname{diag}\left(\boldsymbol{x}_{t} \boldsymbol{w}_{t}\right)-\boldsymbol{x}_{t+1} \operatorname{diag}\left(\boldsymbol{w}_{t}\right) \boldsymbol{x}_{t}^{\prime}\right)^{-1}\left(\boldsymbol{x}_{t+1} \operatorname{diag}\left(\boldsymbol{w}_{t}\right)\left[\mathbf{1}_{N}+\boldsymbol{x}_{t}^{\prime}\left(\boldsymbol{e}_{t+1}-\mathbf{1}_{K}\right)\right]\right) \succ \mathbf{0}_{K}, \quad \forall t \geq 0,
$$

which is satisfied if for all $t$,

$$
\left\{\begin{array}{cll}
\text { i) } & x_{t, n}^{(k)} \in[0,1), & \forall k \in\{1, \ldots, K\}, \forall n \in\{1, \ldots, N\}, \\
\text { ii) } & 0<\lambda_{n}=\sum_{k=1}^{K} x_{t, n}^{(k)}<1, & \forall n \in\{1, \ldots, N\} \\
\text { iii) } & \sum_{n=1}^{N} x_{t, n}^{(k)} w_{t, n}>0, & \forall k \in\{1, \ldots, K\} .
\end{array}\right.
$$

Whenever the above condition (5) is met, the vector of returns is equal to

$$
\begin{aligned}
\boldsymbol{r}_{t+1} & =\left(\operatorname{diag}\left(\boldsymbol{x}_{t} \boldsymbol{w}_{t}\right)-\boldsymbol{x}_{t+1} \operatorname{diag}\left(\boldsymbol{w}_{t}\right) \boldsymbol{x}_{t}^{\prime}\right)^{-1}\left(\boldsymbol{x}_{t+1} \operatorname{diag}\left(\boldsymbol{w}_{t}\right)\left[\mathbf{1}_{N}+\boldsymbol{x}_{t}^{\prime}\left(\boldsymbol{e}_{t+1}-\mathbf{1}_{K}\right)\right]\right)-\mathbf{1}_{K} \\
& =\left(\operatorname{diag}\left(\boldsymbol{x}_{t} \boldsymbol{\phi}_{t}\right)-\boldsymbol{x}_{t+1} \operatorname{diag}\left(\boldsymbol{\phi}_{t}\right) \boldsymbol{x}_{t}^{\prime}\right)^{-1}\left(\left(\boldsymbol{x}_{t+1}-\boldsymbol{x}_{t}\right) \boldsymbol{\phi}_{t}+\boldsymbol{x}_{t+1} \operatorname{diag}\left(\boldsymbol{\phi}_{t}\right) \boldsymbol{x}_{t}^{\prime} \boldsymbol{e}_{t+1}\right),
\end{aligned}
$$

the growth rate of rescaled wealths $\boldsymbol{\rho}_{t+1}=\operatorname{diag}\left(\boldsymbol{w}_{t}\right)^{-1} \boldsymbol{w}_{t+1}-\mathbf{1}_{N}$ is given by

$$
\boldsymbol{\rho}_{t+1}=\boldsymbol{x}_{t}^{\prime}\left(\boldsymbol{r}_{t+1}+\boldsymbol{e}_{t+1}\right)
$$

and finally, agents' market shares have the following dynamics:

$$
\boldsymbol{\phi}_{t+1}=\operatorname{diag}\left(\boldsymbol{\phi}_{t}\right) \times \frac{\mathbf{1}_{N}+\boldsymbol{x}_{t}^{\prime}\left[\boldsymbol{r}_{t+1}+\boldsymbol{e}_{t+1}\right]}{1+\mathbf{1}_{N}^{\prime} \operatorname{diag}\left(\boldsymbol{\phi}_{t}\right) \boldsymbol{x}_{t}^{\prime}\left[\boldsymbol{r}_{t+1}+\boldsymbol{e}_{t+1}\right]} .
$$

The proof of the proposition, along with all proofs, can be found in the Appendix.

We underline that the restriction (6) can be interpreted as the multi-dimensional equivalent of the condition in Proposition 2.2 in Anufriev et al. (2006). Essentially, the requirement is that all portfolios are long-only (positive demands $(i)$ with no leverage $(i i)$ ), and that the aggregate demand is strictly positive for all assets (iii). While these requirements are quite standard in the literature (see e.g. Assumption 2 in Anufriev et al. (2012)), we will see in Sections 3 and 6.2 that the most interesting (i.e., realistic) configurations are those for which short-selling is allowed. 
The investment functions of the agents have the same properties as those in Anufriev and Bottazzi (2010), that is to say: they are functions of the $L$ past returns. Formally, we write this as: $x_{t, n}^{(k)}=f_{n}^{(k)}\left(\boldsymbol{r}_{t-1}, \ldots, \boldsymbol{r}_{t-L}\right)$. The vector format of all demands will be written as a multivariate function $\boldsymbol{f}_{t}$. A commonplace example is when agents have mean-variance preferences and base their forecasts on sample moments. In this case, demands have the following forms:

$$
\boldsymbol{x}_{t, n}=\frac{1}{\gamma_{n}} \boldsymbol{\Sigma}_{t, n}^{-1} \boldsymbol{\mu}_{t, n}
$$

where $\boldsymbol{\Sigma}_{t, n}$ is agent $n$ 's estimator of the covariance matrix of total returns (price returns plus dividend yield) and $\boldsymbol{\mu}_{t, n}$ is the estimator of the corresponding means. Both estimators are computed with the knowledge of the last $L$ returns. If $L$ is not sufficiently large (i.e., greater than $K$ ), then $\boldsymbol{\Sigma}_{t, n}$ cannot be the sample estimator because it is then singular. We refer to Pantaleo et al. (2011) and Turtle and Wang (2016) for recent discussions on this topic. Finally, $\gamma_{n}>0$ in (10) is the risk aversion parameter of agent $n$ : when it increases, it reduces the leverage implied by the positions taken by agent $n$.

\section{Procedural rationality}

In this section, we define the type of equilibrium for which we will carry our analysis. We then derive the relationships between portfolio compositions $(\boldsymbol{x})$, market shares $(\boldsymbol{\phi})$ and asset returns $(\boldsymbol{r})$ in such equilibria.

\subsection{Fundamental identities}

A vast strand of the literature on HAMs focuses on the fixed points of dynamical systems such as the one described in Proposition 1. These steady states are characterized by constant returns (or prices) and constant market shares. The remaining degree of freedom lies in the choice of portfolio compositions. In this case, agents are allowed to change their beliefs and/or preferences and adjust their investment strategy accordingly (e.g. with respect to past performance for trend-chasers and contrarians). An alternative perspective, proposed by Anufriev and Bottazzi (2010), is to fix the investment fractions and market shares at equilibrium, while letting the asset returns loose. As argued by Anufriev et al. (2012), this second option leaves room for nontrivial correlation patterns among returns and is thus more in line with the stylized behaviors observed on financial markets. While it is clearly out of the scope of the present paper to discuss the theoretical or empirical relevance of each option, we choose to adopt the second approach. Henceforth, equilibria will therefore be defined as follows.

Definition 2. A procedurally consistent equilibrium (PCE) is a trajectory of the system defined by (7) and (9) with fixed investment shares $\boldsymbol{x}_{t}=\boldsymbol{x}^{*}$ and wealth distributions $\boldsymbol{\phi}_{t}=\boldsymbol{\phi}^{*}$.

We start by briefly investigating the case when there is only one agent on the market $(N=1)$. We recall that the case $N=1$ and $K=1$ is solved and discussed in details in Anufriev and Bottazzi (2010). We assume that all demands are nonzero $x^{(k)} \neq 0$ for $k=1, \ldots, K$. Intuitively, an asset with zero demand can simply be removed from the market. The dynamic system then simplifies to

$$
\left\{\begin{array}{rl}
\boldsymbol{x}_{t+1} & =\boldsymbol{f}\left(\boldsymbol{r}_{t, 0}, \ldots, \boldsymbol{r}_{t, L-1}\right) \\
\boldsymbol{r}_{t+1,0} & =\boldsymbol{R}\left(\boldsymbol{f}\left(\boldsymbol{r}_{t, 0}, \ldots, \boldsymbol{r}_{t, L-1}\right), \boldsymbol{x}_{t}, \boldsymbol{e}_{t+1}\right) \\
\boldsymbol{r}_{t+1,1} & =\boldsymbol{r}_{t, 0} \\
\vdots & \\
\boldsymbol{r}_{t+1, L-1} & =\boldsymbol{r}_{t, L-2}
\end{array},\right.
$$


where $\boldsymbol{R}(\boldsymbol{y}, \boldsymbol{x}, \boldsymbol{e})=\left(1+\boldsymbol{x}^{\prime}\left(\boldsymbol{e}-\mathbf{1}_{K}\right)\right)\left(\operatorname{diag}(\boldsymbol{x})-\boldsymbol{y} \boldsymbol{x}^{\prime}\right)^{-1} \boldsymbol{y}-\mathbf{1}_{K}$ (note that the first term $1+\boldsymbol{x}^{\prime}\left(\boldsymbol{e}-\mathbf{1}_{K}\right)$ is a constant). We write $\boldsymbol{r}_{t, l}$ for the vector of returns at time $t-l$.

Given the notorious complexity stemming from the random dividend yields $\boldsymbol{e}_{t}$, it is customary to carry an analysis in which these yields are fixed to their (positive) average value $\overline{\boldsymbol{e}}$ which satisfies $\overline{\boldsymbol{e}} \neq \mathbf{1}_{K}$ and $\overline{\boldsymbol{e}} \succ \mathbf{0}_{K}$. This yields the so-called deterministic skeleton of the system. Now, if agents' investment choices are fixed to $\boldsymbol{x}$ as is required in the definition of PCE, then returns are also frozen because $\boldsymbol{R}(\boldsymbol{x}, \boldsymbol{x}, \overline{\boldsymbol{e}})$ remains constant. Consequently, we will omit the time dependence in the subscripts as often as possible to ease the notations.

The following lemma shows that when there is only one investor, all returns are equal (the assets can of course still be distinguished by their total returns if their dividend yields differ).

Lemma 3. If $N=1$, then at a PCE, the returns of the $K$ assets $\boldsymbol{r}^{*}$ and the demands $\boldsymbol{x}^{*}$ satisfy the following vector identity

$$
\boldsymbol{r}^{*}=\frac{\overline{\boldsymbol{e}}^{\prime} \boldsymbol{x}^{*}}{1-\mathbf{1}_{K}^{\prime} \boldsymbol{x}^{*}} \mathbf{1}_{K}
$$

Note that the returns are all positive when condition (6) $i$ i) is satisfied. When the demands sum to one, the equilibrium returns are not finite and when the investor uses leverage (i.e., $\mathbf{1}_{K}^{\prime} \boldsymbol{x}^{*}>1$ ), the returns are negative. When there is only one asset, there is a one-to-one relationship between $x^{*}$ and $r^{*}$ (the so-called Equilibrium Market Curve; see Proposition 3.1 in Anufriev and Bottazzi (2010)). When many assets are available, there are an infinite number of demand vectors $\boldsymbol{x}^{*}$ that yield the same return $r^{*}$.

We now turn to the general case with $N$ investors and in this case the system is

$$
\left\{\begin{aligned}
\boldsymbol{x}_{t+1} & =\boldsymbol{f}\left(\boldsymbol{r}_{t, 0}, \ldots, \boldsymbol{r}_{t, L-1}\right), \\
\boldsymbol{\phi}_{t+1} & =\boldsymbol{\Phi}\left(\boldsymbol{x}_{\boldsymbol{t}}, \boldsymbol{\phi}_{t}, \boldsymbol{e}_{t+1}, \boldsymbol{R}\left(\boldsymbol{f}\left(\boldsymbol{r}_{t, 0}, \ldots, \boldsymbol{r}_{t, L-1}\right), \boldsymbol{x}_{t}, \phi_{t}, \boldsymbol{e}_{t+1}\right)\right) \\
\boldsymbol{r}_{t+1,0} & =\boldsymbol{R}\left(\boldsymbol{f}\left(\boldsymbol{r}_{t, 0}, \ldots, \boldsymbol{r}_{t, L-1}\right), \boldsymbol{x}_{t}, \boldsymbol{\phi}_{t}, \boldsymbol{e}_{t+1}\right) \\
\boldsymbol{r}_{t+1,1} & =\boldsymbol{r}_{t, 0} \\
\vdots & \\
\boldsymbol{r}_{t+1, L-1} & =\boldsymbol{r}_{t, L-2}
\end{aligned}\right.
$$

where, by Proposition 1 ,

$$
\begin{aligned}
& \boldsymbol{\Phi}(\boldsymbol{x}, \boldsymbol{\phi}, \boldsymbol{e}, \boldsymbol{r})=\operatorname{diag}(\boldsymbol{\phi}) \times \frac{\mathbf{1}_{N}+\boldsymbol{x}^{\prime}(\boldsymbol{r}+\boldsymbol{e})}{1+\mathbf{1}_{N}^{\prime} \operatorname{diag}(\boldsymbol{\phi}) \boldsymbol{x}^{\prime}(\boldsymbol{r}+\boldsymbol{e})}, \\
& \boldsymbol{R}(\boldsymbol{y}, \boldsymbol{x}, \boldsymbol{\phi}, \boldsymbol{e})=\left(\operatorname{diag}(\boldsymbol{x} \phi)-\boldsymbol{y} \operatorname{diag}(\boldsymbol{\phi}) \boldsymbol{x}^{\prime}\right)^{-1}\left((\boldsymbol{y}-\boldsymbol{x}) \boldsymbol{\phi}+\boldsymbol{y} \operatorname{diag}(\boldsymbol{\phi}) \boldsymbol{x}^{\prime} \boldsymbol{e}\right) .
\end{aligned}
$$

Just as in the case with only one agent, invariant $\boldsymbol{\phi}$ and $\boldsymbol{x}$ imply constant returns when the dividend yields are fixed to $\overline{\boldsymbol{e}}$. We start by tackling a trivial case with an important assumption. For notational conciseness, we introduce the aggregate (market) demand of asset $k: z^{(k)}=\sum_{n=1}^{N} \phi_{n} x_{n}^{(k)}$ and the corresponding vector is denoted by $\boldsymbol{z}$. A crucial assumption is the following.

(A) The aggregate demands are not all equal to zero: $\boldsymbol{z}=\boldsymbol{x} \boldsymbol{\phi} \neq \mathbf{0}_{K}$.

In the case when all $z^{(k)}$ are null, the equilibrium can only be reached when $\boldsymbol{r}=-\overline{\boldsymbol{e}}$. This case is somewhat similar to that of Proposition 4.1 (iii) of Anufriev and Bottazzi (2010) in which the aggregate demand for the asset is also equal to zero. The distribution of market shares can then have any form (the $\phi_{n}$ need only to be positive and sum to one). Henceforth, we assume that assumption (A) holds. 


\subsection{Nonzero aggregate demands}

We now consider two configurations. In the first one, all $z^{(k)}$ are nonzero (strictly positive or negative) and in the second one (in the next subsection), $z^{(k)}=0$ for at least one $k$ (and up to $K-1$ ). Surprisingly, the following proposition shows that when none of the $z^{(k)}$ are equal to zero, the equilibrium price returns are uniform.

Proposition 4. No zero aggregate demand. Assume $z^{(k)} \neq 0$ for all $k \in\{1, \ldots, K\}$. At a $P C E$, there can be $N^{\diamond} \in\{1, \ldots, N\}$ surviving agents (i.e., with strictly positive market share). We denote the vector of their market shares $\boldsymbol{\phi}^{\diamond} \succ \mathbf{0}_{N^{\diamond}}$. If their demand matrix, which is a subset of $\boldsymbol{x}$, is written $\boldsymbol{x}^{\diamond}$, then it must satisfy the following relationship:

$$
\boldsymbol{r}^{*}=\frac{\left(\phi^{\diamond}\right)^{\prime}\left(\boldsymbol{x}^{\diamond}\right)^{\prime} \overline{\boldsymbol{e}}}{1-\left(\boldsymbol{\phi}^{\diamond}\right)^{\prime}\left(\boldsymbol{x}^{\diamond}\right)^{\prime} \mathbf{1}_{K}} \mathbf{1}_{K}
$$

We highlight that under condition $i i i$ ) in 6) demands are strictly positive and under $i i$ ), the returns are positive at equilibrium. A nice property at equilibrium is that the weighted average of individual total performance can be shown to be equal to the (unique) return of assets:

$$
\left(\boldsymbol{\phi}^{\diamond}\right)^{\prime}\left(\boldsymbol{x}^{\diamond}\right)^{\prime}\left(\boldsymbol{r}^{*}+\overline{\boldsymbol{e}}\right)=\frac{\left(\boldsymbol{\phi}^{\diamond}\right)^{\prime}\left(\boldsymbol{x}^{\diamond}\right)^{\prime} \overline{\boldsymbol{e}}}{1-\left(\boldsymbol{\phi}^{\diamond}\right)^{\prime}\left(\boldsymbol{x}^{\diamond}\right)^{\prime} \mathbf{1}_{K}}=r^{*} .
$$

and the growths of rescaled wealths $\left(\boldsymbol{x}^{\diamond}\right)^{\prime}\left(\boldsymbol{r}^{*}+\overline{\boldsymbol{e}}\right)$ are all equal to this value.

There are many degrees of freedom embedded in Proposition $4 .{ }^{4}$ Let us consider the market as a whole. $\phi^{*}$ denotes all market shares ( $\phi^{\diamond}$ concatenated with additional zeros) and $\boldsymbol{x}^{*}$ stands for all asset demands. Then of course the completed version of Equation (14)

$$
\boldsymbol{r}^{*}=\frac{\left(\boldsymbol{\phi}^{*}\right)^{\prime}\left(\boldsymbol{x}^{*}\right)^{\prime} \overline{\boldsymbol{e}}}{1-\left(\boldsymbol{\phi}^{*}\right)^{\prime}\left(\boldsymbol{x}^{*}\right)^{\prime} \mathbf{1}_{K}} \mathbf{1}_{K}=\frac{\left(\boldsymbol{z}^{*}\right)^{\prime} \overline{\boldsymbol{e}}}{1-\left(\boldsymbol{z}^{*}\right)^{\prime} \mathbf{1}_{K}} \mathbf{1}_{K}=r^{*} \mathbf{1}_{K} .
$$

is also true. The demands of the vanishing agents are simply not taken into account. The progressive disappearance of agents depending on their portfolio choice is an captivating topic and we initiate a discussion on this topic in Section 5.

Both forms (14) and (16) naturally share strong similarities with the simplified version (12). More surprisingly, their expressions also recall those obtained in Anufriev et al. (2012), in which the model assumptions are slightly different. ${ }^{5}$ In equation (3.5) of Anufriev et al. (2012), the expected return at equilibrium is equal to the sum of two terms. The first one is the risk-free rate; the second one is the market-weighted average of dividend yields divided by the aggregate position in the risk-less asset. This second term is equivalent to the constant term $r^{*}$ in (16). Another close similarity with the result of Anufriev et al. (2012) is that equilibrium returns are all equal in the cross-section at the PCE. We nevertheless underline that this is a strong requirement which is obviously not in line with returns observed on financial markets. As we will show subsequently, more general patterns emerge when some aggregate demands vanish.

The impact of aggregate demands on the equilibrium return $r^{*}$ is worth commenting. The second equality in (16) characterizes this effect. The equivalence

$$
\frac{\partial r^{*}}{\partial z^{(j)}}=\frac{\bar{e}^{(j)}\left(1-\left(\boldsymbol{z}^{*}\right)^{\prime} \mathbf{1}_{K}\right)+\left(\boldsymbol{z}^{*}\right)^{\prime} \overline{\boldsymbol{e}}}{\left(1-\left(\boldsymbol{z}^{*}\right)^{\prime} \mathbf{1}_{K}\right)^{2}} \geq 0 \quad \Leftrightarrow \quad \bar{e}_{j} \geq-r^{*}
$$

\footnotetext{
${ }^{4}$ This is also the case with only one asset, as is shown in Corollaries 4.1 and 4.2 of Anufriev and Bottazzi (2010).

${ }^{5}$ If only because the risk-free asset remains endogenous throughout their article, while we get rid of it via the normalizations in (3).
} 
is in fact deceptive, because $r^{*}$ is most often increasing in $z^{(j)}$. More precisely, the equivalence in (17) implies that the equilibrium return is increasing in $z^{(j)}$ as long as the total return of the $j^{\text {th }}$ asset is positive. But given the singularity stemming from the denominator, $r^{*}$ is increasing in $z^{(j)}$ to $+\infty$ to the left of the singularity and increasing from $-\infty$ to the right of the singularity. Accordingly, investors should invest as much as they can in high-dividend yield assets, with a limit such that the global aggregate demand is strictly smaller than one. This is an insightful constraint on the source of leverage that investors should use. If an investor is willing to invest a large share of his wealth (possibly even more than he can afford), then he can either resort to debt (via the risk-free asset) or short-selling. The requirement that $1-\left(\boldsymbol{z}^{*}\right)^{\prime} \mathbf{1}_{K}>0$, which ensures both positive returns and wealth growth, implies that agents should ultimately rely more on the short-selling of some assets (and not on debt) to finance possibly large positions in other assets. ${ }^{6}$ There is nevertheless a limit to short-selling: the long positions must overall offset the short ones so that the numerator in (16) remains positive.

We end this discussion with one last comment. The most striking and counter-intuitive feature of the model is that it allows assets to have demands with opposite signs while having equal price returns. This stands in contrast with the basic economic mechanism that pushes prices up (resp. down) when the net demand is positive (resp. negative). Fortunately, we switch below the more challenging case when some demands $z^{(k)}$ can vanish and in this case the relationships between demands and returns are far more intricate.

\subsection{Some zero aggregate demands}

We now turn to the case where some assets face zero aggregate demands. Obviously, this requires that condition (6) be violated. We write $\boldsymbol{r}^{ \pm}$for the equilibrium vector of returns for the $K^{ \pm}$assets with nonzero demand and $\boldsymbol{r}^{0}$ for the vector of returns for the $K^{0}$ assets with zero demands (obviously, $K=K^{ \pm}+K^{0}$ ). The corresponding demand matrices are denoted by $\boldsymbol{x}^{ \pm}$and $\boldsymbol{x}^{0}$. In sum, we decompose the basic elements of our study into

$$
\boldsymbol{x}^{*}=\left(\begin{array}{c}
\boldsymbol{x}^{ \pm} \\
\boldsymbol{x}^{0}
\end{array}\right) \quad, \quad \boldsymbol{r}^{*}=\left(\begin{array}{c}
\boldsymbol{r}^{ \pm} \\
\boldsymbol{r}^{0}
\end{array}\right) \quad \text { and } \quad \overline{\boldsymbol{e}}^{*}=\left(\begin{array}{c}
\overline{\boldsymbol{e}}^{ \pm} \\
\overline{\boldsymbol{e}}^{0}
\end{array}\right)
$$

To preserve consistency with our previous conventions, we write $x_{i}^{0, j}$ for the value located at the $j^{\text {th }}$ row and $i^{\text {th }}$ column of $\boldsymbol{x}^{0}$. We proceed similarly with the other vectors and matrices. Additionally, we introduce the following notation:

$$
\boldsymbol{\Lambda}^{*}=\boldsymbol{x}^{*} \operatorname{diag}\left(\boldsymbol{\phi}^{*}\right)\left(\boldsymbol{x}^{*}\right)^{\prime}=\left(\begin{array}{ll}
\boldsymbol{\Lambda}^{ \pm} & \boldsymbol{\Lambda}^{ \pm, 0} \\
\boldsymbol{\Lambda}^{0, \pm} & \boldsymbol{\Lambda}^{0}
\end{array}\right)
$$

where $\boldsymbol{\Lambda}^{ \pm}, \boldsymbol{\Lambda}^{0}$ and $\boldsymbol{\Lambda}^{ \pm, 0}=\left(\boldsymbol{\Lambda}^{\mathbf{0 , \pm}}\right)^{\prime}$ are respectively $\left(K^{ \pm} \times K^{ \pm}\right),\left(K^{0} \times K^{0}\right)$ and $\left(K^{ \pm}, K^{0}\right)$ matrices. $\Lambda^{*}$ is the matrix of (relative) wealth-weighted products between demands. Said differently, $\boldsymbol{\Lambda}^{*}$ is the collection of wealth-weighted second order cross-moments of demands.

For simplicity, and without any loss of generality, we will assume in the following proposition and in the sequel of the section that all agents survive. In all generality, the number of nonvanishing agents can lie anywhere between 1 and $N$, as in Proposition 4 (the stars and diamonds for $\boldsymbol{\phi}$ and $\boldsymbol{x}$ can be interchanged as in Equations (14) and (16)).

\footnotetext{
${ }^{6}$ It seems unlikely that regulatory institutions, such as the Bank of International Settlements, would advocate or encourage such a strategy.
} 
Proposition 5. At least one zero aggregate demand. With the notations used above, the first $K^{ \pm}$equilibrium returns are all equal, similarly as in Proposition 4:

$$
\boldsymbol{r}^{ \pm}=\frac{\left(\phi^{*}\right)^{\prime}\left(\boldsymbol{x}^{ \pm}\right)^{\prime} \overline{\boldsymbol{e}}^{ \pm}}{1-\left(\phi^{*}\right)^{\prime}\left(\boldsymbol{x}^{ \pm}\right)^{\prime} \mathbf{1}_{K^{ \pm}}} \mathbf{1}_{K^{ \pm}}=\frac{\left(\phi^{*}\right)^{\prime}\left(\boldsymbol{x}^{*}\right)^{\prime} \overline{\boldsymbol{e}}}{1-\left(\phi^{*}\right)^{\prime}\left(\boldsymbol{x}^{*}\right)^{\prime} \mathbf{1}_{K}} \mathbf{1}_{K^{ \pm}},
$$

and the remaining $K^{0}$ returns satisfy the following relationship:

$$
\boldsymbol{r}^{0}=-\overline{\boldsymbol{e}}^{0}-\left(\boldsymbol{\Lambda}^{0}\right)^{-1} \boldsymbol{\Lambda}^{0, \pm}\left(\boldsymbol{r}^{ \pm}+\overline{\boldsymbol{e}}^{ \pm}\right)
$$

Since we assume that $\operatorname{rank}(\boldsymbol{x})=\min (N, K)$, which in turn implies $\operatorname{rank}\left(\boldsymbol{x}^{0}\right)=\min \left(N, K^{0}\right)$, the existence of $K^{0}$ zero demands requires $N>K^{0}$ and hence $\operatorname{rank}\left(\boldsymbol{\Lambda}^{0}\right)=K^{0}$ so that its inverse is well defined in (20). The expression for $\boldsymbol{r}^{ \pm}$is the same as that of $\boldsymbol{r}^{*}$ and we need not further comment on it.

On the other hand, the identity (20) is not straightforward to interpret, but we can rewrite it in an alternative form. First of all, the components of the equation can be decomposed as $\boldsymbol{\Lambda}^{0}=\boldsymbol{x}^{0} \operatorname{diag}\left(\boldsymbol{\phi}^{*}\right)\left(\boldsymbol{x}^{0}\right)^{\prime}$ and $\boldsymbol{\Lambda}^{0, \pm}=\boldsymbol{x}^{0} \operatorname{diag}\left(\boldsymbol{\phi}^{*}\right)\left(\boldsymbol{x}^{ \pm}\right)^{\prime}$. Second, there is no reason a priori that assets should have exactly the same return (it is never the case in practice). So let us assume until the end of the subsection that $K^{ \pm}=1$, so that the returns are likely to be all different. Simple algebra ${ }^{7}$ provides a new form of $(20)$ :

$$
\begin{aligned}
\frac{\boldsymbol{r}^{0}+\overline{\boldsymbol{e}}^{0}}{r^{ \pm}+\bar{e}^{ \pm}} & =\underset{\boldsymbol{y} \in \mathbb{R}^{K^{0}}}{\operatorname{argmin}}\left\{\left(\boldsymbol{y}+\boldsymbol{\Lambda}^{\mathbf{0}, \pm}\right)^{\prime} \boldsymbol{\Lambda}^{0}\left(\boldsymbol{y}+\boldsymbol{\Lambda}^{0, \pm}\right)\right\} \\
& =\underset{\boldsymbol{y} \in \mathbb{R}^{K^{0}}}{\operatorname{argmin}}\left\{\left(\boldsymbol{x}^{ \pm}+\boldsymbol{y}^{\prime} \boldsymbol{x}^{0}\right) \operatorname{diag}\left(\boldsymbol{\phi}^{*}\right)\left(\boldsymbol{x}^{ \pm}+\boldsymbol{y}^{\prime} \boldsymbol{x}^{0}\right)^{\prime}\right\}
\end{aligned}
$$

This identity implies that, given $r^{ \pm}, \boldsymbol{r}^{0}$ is such that

$$
d=\left(\boldsymbol{r}^{*}+\overline{\boldsymbol{e}}\right)^{\prime} \boldsymbol{x}^{*} \operatorname{diag}\left(\boldsymbol{\phi}^{*}\right)\left(\boldsymbol{x}^{*}\right)^{\prime}\left(\boldsymbol{r}^{*}+\overline{\boldsymbol{e}}\right)=\left(\boldsymbol{\rho}^{*}\right)^{\prime} \operatorname{diag}\left(\boldsymbol{\phi}^{*}\right) \boldsymbol{\rho}^{*}
$$

is minimal (the vector of wealths' growth $\boldsymbol{\rho}$ is defined in (8)). By the definition of a PCE, it must hold that $\boldsymbol{\rho}^{*}=\rho \mathbf{1}_{N}$. Hence, $d=\rho^{2}$ and the returns of those assets with zero aggregate demand are such that the squared growth of wealth is minimal. This is a surprising feature of the model. Assuming that the growth rate of wealth is positive, this indicates that the equilibrium mechanism minimizes the profitability for investors.

\subsection{An identity with some zero demand assets}

We now try to characterize the dependence between the total returns of assets when they can face zero aggregate demand. As before, we assume $K^{ \pm}=1$ so that assets are likely to have different equilibrium returns. We focus on assets with zero aggregate demands because an analysis for $r^{ \pm}$is already detailed in Section 3.2. We will refer to the assets with zero demand as the ZD assets.

\footnotetext{
${ }^{7}$ The second equality can be viewed as the computation of the estimator of generalized least squares regressions:

$$
\underset{\boldsymbol{b}}{\operatorname{argmin}}(\boldsymbol{y}-\boldsymbol{X} \boldsymbol{b})^{\prime} \boldsymbol{A}(\boldsymbol{y}-\boldsymbol{X} \boldsymbol{b})=\left(\boldsymbol{X}^{\prime} \boldsymbol{A} \boldsymbol{X}\right)^{-1} \boldsymbol{X}^{\prime} \boldsymbol{A} \boldsymbol{y},
$$
}

see Chapter 9 in Greene (2000). 
Rewriting equation (20) as $\boldsymbol{\Lambda}^{0}\left(\boldsymbol{r}^{0}+\overline{\boldsymbol{e}}^{0}\right)=-\boldsymbol{\Lambda}^{0, \pm}\left(r^{ \pm}+\bar{e}^{ \pm}\right)$, we extract the $i^{\text {th }}$ value:

$$
\begin{aligned}
\sum_{j=1}^{K-1} \Lambda_{i, j}^{0}\left(r^{0,(j)}+\bar{e}^{0,(j)}\right) & =-\Lambda_{i}^{0, \pm}\left(r^{ \pm}+\bar{e}^{ \pm}\right) \\
\Leftrightarrow \quad\left(r^{0,(i)}+\bar{e}^{0,(i)}\right) & =-\frac{1}{\Lambda_{i, i}^{0}}\left[\Lambda_{i}^{0, \pm}\left(r^{ \pm}+\bar{e}^{ \pm}\right)+\sum_{j \neq i} \Lambda_{i, j}^{0}\left(r^{0,(j)}+\bar{e}^{0,(j)}\right)\right] \\
& =-\frac{1}{\Lambda_{i, i}^{0}} \sum_{n=1}^{N} \phi_{n} x_{n}^{0, i} \underbrace{\left[x_{n}^{ \pm}\left(r^{ \pm}+\bar{e}^{ \pm}\right)+\sum_{j \neq i} x_{n}^{0, j}\left(r^{0,(j)}+\bar{e}^{0,(j)}\right)\right]}_{\text {Demand-weighted total returns of other assets }} .
\end{aligned}
$$

At a PCE, a ZD asset's total return is thus equal to a simple linear combination of all other assets' total returns. The coefficients of the linear combination depend on $\boldsymbol{\Lambda}^{*}$ solely. We recall that in equation (23), the values $\Lambda_{i, i}^{0}$ are always positive, therefore the sign of the relationship between two ZD assets is entirely driven by $\Lambda_{i, j}^{0}$.

In a dynamic setting, the economic mechanisms are much more complicated, but as the convergence to the equilibrium nears, the above relationship becomes more and more salient. We provide an illustration of the equation (23) at work with an example in Section 6.2.

With regard to market selection in the presence of ZD assets, it is not possible to factor out market shares in the final computations and this prevents us from deriving simple analytical results such as those in Section 5. As we will show in the Section 6.2, the same strategy can lead to survivorship or to disappearance, depending on the initial market shares.

\section{System dynamics}

In this section we work with constant portfolio composition $\boldsymbol{x}$ and dividend yields $\overline{\boldsymbol{e}}$ so that the system describing the evolution of price returns and market shares becomes endogenous. We formalize the discrete evolution operator and then study the system dynamics in terms of its Jacobian matrix. In this section we consider that condition (6) is met.

\subsection{Discrete evolution of the system}

At time $t \geq 0$, the current state of the system is written $\boldsymbol{S}_{t}=\left(r_{t}^{(1)}, \ldots, r_{t}^{(K)} ; \phi_{t, 1}, \ldots, \phi_{t, N}\right)$. The system has $K+N-1$ independent variables because $\sum_{n=1}^{N} \phi_{t, n}=1$, but we work with the $K+N$ dimensional representation, as in Anufriev and Dindo (2010). It evolves in the domain $\mathcal{D}=(-1,+\infty)^{K} \times \Delta_{N}$, with $\Delta_{N}$ the simplex of market shares

$$
\Delta_{N}=\left\{\left(\phi_{1}, \ldots, \phi_{N}\right) ; \sum_{n=1}^{N} \phi_{n}=1, \quad \phi_{n} \geq 0 \quad \text { for } n=1, \ldots, N\right\} .
$$

The dynamics of the system is given by the explicit evolution operator $\boldsymbol{G}$, defined on $\mathcal{D}$ and such that $\boldsymbol{S}_{t+1}=\boldsymbol{G}\left(\boldsymbol{S}_{t}\right)$. Using Equation (13), the operator $G$ is written as

$$
\boldsymbol{G}:\left\{\begin{array}{l}
\boldsymbol{r}_{t+1}=\left(\operatorname{diag}\left(\boldsymbol{x} \boldsymbol{\phi}_{t}\right)-\boldsymbol{x} \operatorname{diag}\left(\boldsymbol{\phi}_{t}\right) \boldsymbol{x}^{\prime}\right)^{-1}\left(\boldsymbol{x} \operatorname{diag}\left(\boldsymbol{\phi}_{t}\right) \boldsymbol{x}^{\prime} \overline{\boldsymbol{e}}\right) \\
\boldsymbol{\phi}_{t+1}=\operatorname{diag}\left(\boldsymbol{\phi}_{t}\right) \times \frac{\mathbf{1}_{N}+\boldsymbol{x}^{\prime}\left(\boldsymbol{r}_{t+1}+\overline{\boldsymbol{e}}\right)}{1+\mathbf{1}_{N}^{\prime} \operatorname{diag}\left(\boldsymbol{\phi}_{t}\right) \boldsymbol{x}^{\prime}\left(\boldsymbol{r}_{t+1}+\overline{\boldsymbol{e}}\right)}
\end{array}\right.
$$

or, with $\boldsymbol{\Phi}$ and $\boldsymbol{R}$, as

$$
\boldsymbol{G}:\left\{\begin{aligned}
\boldsymbol{r}_{t+1} & =\boldsymbol{R}\left(\boldsymbol{x}, \boldsymbol{x}, \phi_{t}, \overline{\boldsymbol{e}}\right) \\
\boldsymbol{\phi}_{t+1} & =\boldsymbol{\Phi}\left(\boldsymbol{x}, \boldsymbol{\phi}_{t}, \overline{\boldsymbol{e}}, \boldsymbol{r}_{t+1}\right)
\end{aligned}\right.
$$


The deterministic and discrete dynamic system under scrutiny is denoted by $\left(\boldsymbol{S}_{0}, \boldsymbol{G}\right)$ and has parameters $(\boldsymbol{x}, \overline{\boldsymbol{e}})$. As we work under condition (6), it evolves within $\mathcal{D}$ provided that $\boldsymbol{S}_{0} \in \mathcal{D}$. Indeed, if the system state at time $t$ is in $\mathcal{D}$, prices are positive at time $t+1$ because we work under condition (6). This, in turn, ensures that the returns at time $t+1$ are greater than -1 so that $\boldsymbol{r}_{t+1} \in(-1,+\infty)^{K}$. Because prices stay positive, and agents cannot go short under condition (6), the agent wealths stay non-negative which ensures that the vector of market shares $\phi_{t+1}$ belongs to the simplex $\Delta_{N}$.

We denote by $\boldsymbol{S}^{*}$ a steady state equilibrium of this system, that is $\boldsymbol{S}^{*}$ such that $\boldsymbol{S}^{*}=\boldsymbol{G}\left(\boldsymbol{S}^{*}\right)$. The vertices of the simplex $\Delta_{N}$ are obvious steady states with a single survivor.

\subsection{The Jacobian matrix of the system}

The Jacobian matrix of the system is denoted by $\boldsymbol{J}$. It can be written as a $(K+N) \times(K+N)$ block matrix:

$$
\boldsymbol{J}=\left(\begin{array}{cc}
\mathcal{R} & \mathcal{T} \\
\mathcal{H} & \mathcal{F}
\end{array}\right)
$$

where $\mathcal{R}=\frac{\partial \boldsymbol{r}_{t+1}}{\partial \boldsymbol{r}_{t}}, \mathcal{T}=\frac{\partial \boldsymbol{r}_{t+1}}{\partial \boldsymbol{\phi}_{t}}, \mathcal{H}=\frac{\partial \phi_{t+1}}{\partial \boldsymbol{r}_{t}}$ and $\mathcal{F}=\frac{\partial \phi_{t+1}}{\partial \phi_{t}}$. And the blocks $\mathcal{R}, \mathcal{T}, \mathcal{H}$ and $\mathcal{F}$ have respective sizes $K \times K, K \times N, N \times K$ and $N \times N$.

In the proposition below, we give the expressions to compute the elements of each block of $\boldsymbol{J}$ in the general case.

Proposition 6. The elements of the Jacobian matrix $\boldsymbol{J}$ have expressions, for $k, i=1, \ldots, K$ and $n, q=1, \ldots, N$,

$$
\begin{aligned}
& \frac{\partial r_{t+1}^{(k)}}{\partial r_{t}^{(i)}}=0, \quad \frac{\partial \phi_{t+1, n}}{\partial r_{t}^{(k)}}=0, \quad \frac{\partial r_{t+1}^{(k)}}{\partial \phi_{t, n}}=\sum_{i=1}^{K} d_{k i}\left(\sum_{l=1}^{K} x_{n}^{(i)} x_{n}^{(l)}\left(r_{t+1}^{(l)}+\bar{e}^{(l)}\right)-x_{n}^{(l)} r_{t+1}^{(l)}\right) \\
& \frac{\partial \phi_{t+1, n}}{\partial \phi_{t, q}}=\frac{1+\sum_{k=1}^{K} x_{n}^{(k)}\left(r_{t+1}^{(k)}+\bar{e}^{(k)}\right)}{1+\sum_{v=1}^{N} \sum_{k=1}^{K} \phi_{t, v} x_{v}^{(k)}\left(r_{t+1}^{(k)}+\bar{e}^{(k)}\right)}\left(\delta_{n q}-\phi_{t, n} \frac{\sum_{k=1}^{K} x_{q}^{(k)}\left(r_{t+1}^{(k)}+\bar{e}^{(k)}\right)}{1+\sum_{v=1}^{N} \sum_{k=1}^{K} \phi_{t, v} x_{v}^{(k)}\left(r_{t+1}^{(k)}+\bar{e}^{(k)}\right)}\right) \\
& +\phi_{t, n} \sum_{k=1}^{K}\left[\frac{x_{n}^{(k)}\left(1+\sum_{v=1}^{N} \sum_{l=1}^{K} \phi_{t, v} x_{v}^{(l)}\left(r_{t+1}^{(l)}+\bar{e}^{(l)}\right)\right)-\left(\sum_{v=1}^{N} \phi_{t, v} x_{v}^{(k)}\right)\left(1+\sum_{l=1}^{K} x_{n}^{(l)}\left(r_{t+1}^{(l)}+\bar{e}^{(l)}\right)\right)}{\left(1+\sum_{v=1}^{N} \sum_{l=1}^{K} \phi_{t, v} x_{v}^{(l)}\left(r_{t+1}^{(l)}+\bar{e}^{(l)}\right)\right)^{2}}\right. \\
& \left.\quad \times \sum_{i=1}^{K} d_{k i}\left(\sum_{l=1}^{K} x_{q}^{(i)} x_{q}^{(l)}\left(r_{t+1}^{(l)}+\bar{e}^{(l)}\right)-x_{q}^{(l)} r_{t+1}^{(l)}\right)\right]
\end{aligned}
$$

where $\boldsymbol{D}=\left(\operatorname{diag}\left(\boldsymbol{x} \phi_{t}\right)-\boldsymbol{x} \operatorname{diag}\left(\boldsymbol{\phi}_{t}\right) \boldsymbol{x}^{\prime}\right)^{-1}$ is a $K \times K$ matrix with elements denoted by $d_{k i}$ and $\delta_{n q}$ is the Kronecker delta: $\delta_{n q}=1$ if $n=q$ and $\delta_{n q}=0$ otherwise.

We now have expressions for the elements of the blocks to form the Jacobian matrix $\boldsymbol{J}$. The only numerical computation to be performed is the matrix inversion to obtain $\boldsymbol{D}$. The other expressions are tedious but straightforward to compute.

We now consider the expression of the Jacobian matrix at a fixed-point equilibrium $\boldsymbol{S}^{*}=$ $\left(r^{*}, \ldots, r^{*} ; \phi_{1}^{*}, \ldots, \phi_{N}^{*}\right)$ of the system. It is denoted by $\boldsymbol{J}\left(\boldsymbol{S}^{*}\right)$ with the following block structure

$$
\boldsymbol{J}\left(\boldsymbol{S}^{*}\right)=\left(\begin{array}{cc}
\mathcal{R}\left(\boldsymbol{S}^{*}\right) & \mathcal{T}\left(\boldsymbol{S}^{*}\right) \\
\mathcal{H}\left(\boldsymbol{S}^{*}\right) & \mathcal{F}\left(\boldsymbol{S}^{*}\right)
\end{array}\right)
$$


Proposition 7. At a fixed-point equilibirum $\boldsymbol{S}^{*}$, the elements of the Jacobian matrix $\boldsymbol{J}\left(\boldsymbol{S}^{*}\right)$ have expressions given below, for $k, i=1, \ldots, K$ and $n, q=1, \ldots, N$,

$$
\begin{aligned}
\frac{\partial r_{t+1}^{(k)}}{\partial r_{t}^{(i)}}\left(\boldsymbol{S}^{*}\right) & =0, \quad \frac{\partial \phi_{t+1, n}}{\partial r_{t}^{(k)}}\left(\boldsymbol{S}^{*}\right)=0, \quad \frac{\partial r_{t+1}^{(k)}}{\partial \phi_{t, n}}\left(\boldsymbol{S}^{*}\right)=-r^{*} \sum_{i=1}^{K} d_{k i} \sum_{\substack{l=1 \\
l \neq i}}^{K} x_{n}^{(l)}, \\
\frac{\partial \phi_{t+1, n}}{\partial \phi_{t, q}}\left(\boldsymbol{S}^{*}\right) & =\delta_{n q}-\frac{r^{*} \phi_{n}^{*}}{1+r^{*}}\left[1+\sum_{k=1}^{K}\left(x_{n}^{(k)}-\sum_{v=1}^{N} \phi_{v}^{*} x_{v}^{(k)}\right) \sum_{i=1}^{K} d_{k i} \sum_{\substack{l=1 \\
l \neq i}}^{K} x_{q}^{(l)}\right],
\end{aligned}
$$

where the $d_{k i}$ are the elements of the matrix $\boldsymbol{D}^{*}=\left(\operatorname{diag}\left(\boldsymbol{x} \boldsymbol{\phi}^{*}\right)-\boldsymbol{x} \operatorname{diag}\left(\boldsymbol{\phi}^{*}\right) \boldsymbol{x}^{\prime}\right)^{-1}$.

\subsection{Characteristic polynomial}

The Jacobian matrix $\boldsymbol{J}$ corresponds to the linearization of our discrete non-linear system at its current state, and allows one to grasp its dynamics. At a fixed-point $\boldsymbol{S}^{*}$, the roots of the characteristic polynomial - or eigenvalues - of $\boldsymbol{J}\left(\boldsymbol{S}^{*}\right)$ can be used to determine the stability of the system. The idea is that if the spectral radius of $\boldsymbol{J}\left(\boldsymbol{S}^{*}\right)$, denoted by $\varrho\left(\boldsymbol{J}\left(\boldsymbol{S}^{*}\right)\right)$, is strictly smaller than one then the considered fixed-point is stable. The spectral radius of a matrix is the max of the modulus of its eigenvalues

$$
\varrho\left(\boldsymbol{J}\left(\boldsymbol{S}^{*}\right)\right)=\max _{n}\left\{\left|\zeta_{n}\right|\right\},
$$

where the $\zeta_{n}$ are the eigenvalues of the Jacobian matrix. We refer, among others, to Medio and Lines (2001) for a detailed presentation of the stability analysis of discrete nonlinear systems by means of their Jacobian matrix. At equilibrium, the Jacobian matrix has the following block structure

$$
\boldsymbol{J}\left(\boldsymbol{S}^{*}\right)=\left(\begin{array}{cc}
0_{K, K} & \mathcal{T}\left(\boldsymbol{S}^{*}\right) \\
0_{N, K} & \mathcal{F}\left(\boldsymbol{S}^{*}\right)
\end{array}\right),
$$

with the elements of the blocks $\mathcal{T}\left(\boldsymbol{S}^{*}\right)$ and $\mathcal{F}\left(\boldsymbol{S}^{*}\right)$ given in Proposition 7 . With this block structure, the characteristic polynomial of $\boldsymbol{J}\left(\boldsymbol{S}^{*}\right)$ is written, for $\zeta \in \mathbb{C}$

$$
P_{J}(\zeta)=\operatorname{det}\left(\boldsymbol{J}\left(\boldsymbol{S}^{*}\right)-\zeta I_{N+K}\right)=\operatorname{det}\left(\begin{array}{cc}
-\zeta I_{K} & \mathcal{T}\left(\boldsymbol{S}^{*}\right) \\
0_{N, K} & \mathcal{F}\left(\boldsymbol{S}^{*}\right)-\zeta I_{N}
\end{array}\right) .
$$

Given the properties of the determinant of a triangular matrix by blocks, see Meyer (2000) (Chapter 6 p. 467), $P_{J}(\zeta)$ is obtained as

$$
\begin{aligned}
P_{J}(\zeta) & =\operatorname{det}\left(-\zeta I_{K}\right) \operatorname{det}\left(\mathcal{F}\left(\boldsymbol{S}^{*}\right)-\zeta I_{N}\right) \\
& =(-\zeta)^{K} \operatorname{det}\left(\mathcal{F}\left(\boldsymbol{S}^{*}\right)-\zeta I_{N}\right) .
\end{aligned}
$$

Zero is a root of $P_{J}(\zeta)$ with multiplicity $K$. This root does not affect the stability of the fixed point. Hence, the stability is driven solely by the spectral radius of $\mathcal{F}\left(\boldsymbol{S}^{*}\right)$. This matrix corresponds to the Jacobian matrix of the market share portion of the system. The expression for $P_{J}(\zeta)$ is in line with the intuition stemming from the expression of the evolution operator $\boldsymbol{G}$, that is: the fixed-point equilibrium is stable if its market share sub-part is stable. We denote by $P_{F}$ the characteristic polynomial of $\mathcal{F}\left(\boldsymbol{S}^{*}\right)$, it is written, for $\zeta \in \mathbb{C}$

$$
P_{F}(\zeta)=\operatorname{det}\left(\mathcal{F}\left(\boldsymbol{S}^{*}\right)-\zeta I_{N}\right)
$$

Given the expression for the elements of $\mathcal{F}\left(\boldsymbol{S}^{*}\right)$ in Proposition 7 , it is unlikely to obtain a workable expression for the characteristic polynomial $P_{F}$ in the general case. 


\subsection{Special cases}

We now examine two special cases of interest. The first is when there is only one survivor at equilibrium (a steady state with a single survivor). This case is important because equilibriums with multiple survivors are unlikely when agents are truly heterogeneous and condition (6) is met. This idea is detailed in Anufriev and Bottazzi (2010) for the single risky asset case and we discuss it in the next section.

When there is only one survivor at a fixed-point equilibrium we can assume, without loss of generality, that the first agent survives, so that we have $\phi_{1}^{*}=1$ and $\phi_{n}^{*}=0$ for $n=2, \ldots, N$. In such a case, the Jacobian matrix obtained in Proposition 7 can be simplied further and the lower right block matrix becomes

$$
\mathcal{F}\left(\boldsymbol{S}^{*}\right)=\left(\begin{array}{ccccc}
\frac{1}{1+r^{*}} & 0 & \ldots & \ldots & 0 \\
\frac{-r^{*}}{1+r^{*}} & 1 & \ddots & & \vdots \\
\vdots & 0 & \ddots & \ddots & \vdots \\
\vdots & \vdots & \ddots & \ddots & 0 \\
\frac{-r^{*}}{1+r^{*}} & 0 & \ldots & 0 & 1
\end{array}\right)
$$

Given the lower triangle structure of this matrix, its characteristic polynomial is written

$$
P_{F}(\zeta)=\left(\frac{1}{1+r^{*}}-\zeta\right)(1-\zeta)^{N-1}
$$

We see that, in such a case, $P_{F}$ has two roots: $\frac{1}{1+r^{*}}$ and 1 with multiplicity $N-1$. As soon as $r^{*}>0$ we have $\frac{1}{1+r^{*}}<1$. The problem arises from 1 that is a multiple root of $P_{F}$ because it means the considered fixed-point is non-hyperbolic and we cannot conclude upon its stability (or unstability) using an analysis based on the Jacobian matrix. That's why here we need to rely on another approach as it is developed in Section 5. For a detailed treatment of the notion of hyperbolic fixed-point and the case of non-hyperbolic fixed-points we refer to Medio and Lines (2001) (Chap. 3 p.76).

The second special case is when there are exactly two agents and two risky assets, that is when $N=K=2$, and the two agents survive at a steady state $\boldsymbol{S}^{*}$. In this case, the stability of the fixed point is driven by the spectral radius of the $2 \times 2$ matrix $\mathcal{F}\left(\boldsymbol{S}^{*}\right)$. Because in the two-dimensional case the eigenvalues of the matrix are known in closed form, it is possible to express the condition on the spectral radius of the matrix in terms of a condition to be fulfilled by the trace and the determinant of this matrix. We refer to Medio and Lines (2001) (Chap. 2 pp. 52 and 53) for the stability analysis of a two-dimensional discrete system. This condition is written

$$
\varrho(\mathcal{F})<1 \Leftrightarrow|\operatorname{tr} \mathcal{F}|-1<\operatorname{det}(\mathcal{F})<1
$$

Figure 1 presents the spectral radius and the stability area for the case $N=K=2$ in terms of the trace and determinant of $\mathcal{F}\left(\boldsymbol{S}^{*}\right)$. The stability area is the interior of the triangle formed by the points with coordinates $(0,-1),(2,1)$ and $(-2,1)$. Sadly, given the complexity of $\mathcal{F}\left(\boldsymbol{S}^{*}\right)$ given in (28), it is impossible to further clarify the constraint (31) in terms of the input variables $\boldsymbol{x}, \boldsymbol{\phi}$ and $\bar{e}$.

\section{Market selection}

For any fixed portfolio compositions $\boldsymbol{x}$, it is possible to recursively compute the dynamics of the system described by Proposition 1 given the trajectory of the dividends $\boldsymbol{e}_{t+1}{ }^{8}$ The corresponding

\footnotetext{
${ }^{8}$ More precisely, this will also require initial market shares and initial prices or returns.
} 




Figure 1: Stability area. We show the spectral radius of $\mathcal{F}$ as a function of its trace and determinant in the two-dimensional case $N=K=2$. The region inside the black triangle is where the spectral radius is smaller than one.

deterministic skeletons can then be interpreted in light of the theoretical results of Section 3 . Henceforth, we set the focus on the key topic of market selection which tries to determine which agents will survive.

\subsection{General results}

When demands are constant, the following lemma shows that the market shares evolve endogenously. They are simply updated with the realizations of dividend yields. Indeed, the market shares are impacted by returns in a way that can be expressed only with the previous market shares, fixed demands and earned dividend yields.

Lemma 8. When demands are constant, the market shares evolve according to

$$
\boldsymbol{\phi}_{t+1}=\operatorname{diag}\left(\boldsymbol{\phi}_{t}\right) \times \frac{\mathbf{1}_{N}+\boldsymbol{x}^{\prime}\left(\operatorname{diag}\left(\boldsymbol{x} \boldsymbol{\phi}_{t}\right)-\boldsymbol{x} \operatorname{diag}\left(\boldsymbol{\phi}_{t}\right) \boldsymbol{x}^{\prime}\right)^{-1} \operatorname{diag}\left(\boldsymbol{x} \boldsymbol{\phi}_{t}\right) \boldsymbol{e}_{t+1}}{1+\mathbf{1}_{N}^{\prime} \operatorname{diag}\left(\boldsymbol{\phi}_{t}\right) \boldsymbol{x}^{\prime}\left(\operatorname{diag}\left(\boldsymbol{x} \boldsymbol{\phi}_{t}\right)-\boldsymbol{x} \operatorname{diag}\left(\boldsymbol{\phi}_{t}\right) \boldsymbol{x}^{\prime}\right)^{-1} \operatorname{diag}\left(\boldsymbol{x} \boldsymbol{\phi}_{t}\right) \boldsymbol{e}_{t+1}} .
$$

As a consequence, aggregate demands evolve according to

$$
\boldsymbol{z}_{t+1}=\operatorname{diag}\left(\boldsymbol{z}_{t}\right) \frac{\boldsymbol{r}_{t+1}+\mathbf{1}_{K}}{1+\boldsymbol{z}_{t}^{\prime}\left(\boldsymbol{r}_{t+1}+\boldsymbol{e}_{t+1}\right)},
$$

and zero aggregate demand is a permanent state for an asset: for any $k$,

$$
z_{t}^{(k)}=0 \quad \Longrightarrow \quad z_{t+1}^{(k)}=0
$$

Unfortunately, the conditions under which the system (32) remains well-behaved (i.e the shares always lie between zero and one) is an open and difficult problem in the general case. It can be easily proven that it is the case under condition (6) because the inverse matrix in the numerator is always defined with positive elements (it is the inverse of an $M$-matrix). When short-selling is allowed, singularities may appear depending on the choice of $\boldsymbol{x}$ and $\overline{\boldsymbol{e}}$, but also on the initial point $\phi_{0}$. As we will see in Section 6.2, the convergence of market shares to their equilibrium value may 
not be achieved through a monotone path. Two necessary conditions for the system to be well behaved are obviously that the matrix $\operatorname{diag}\left(\boldsymbol{x} \phi_{t}\right)-\boldsymbol{x} \operatorname{diag}\left(\boldsymbol{\phi}_{t}\right) \boldsymbol{x}^{\prime}$ be well conditioned and that the denominator be nonzero at all times. When (6) fails, these conditions are not guaranteed and the system can degenerate.

Note that the second part of the lemma provides a direct link between aggregate demand for an asset and its price return. All other things equal, the asset with the highest return will see the absolute value of its demand increase. The formula also has another strong implication. Under reasonable assumptions, the denominator in (33) will always be positive ${ }^{9}$ and clearly, the numerator is also always positive. Hence, aggregate demands never change signs. Thus, as the equilibrium nears, there are three possibilities:

$(\mathrm{P})\left\{\begin{array}{l}\bullet\left[\frac{\boldsymbol{r}_{t+1}+\mathbf{1}_{K}}{1+\boldsymbol{z}_{t}^{\prime}\left(\boldsymbol{r}_{t+1}+\boldsymbol{e}_{t+1}\right)}\right]_{k} \rightarrow 1 \text { and the aggregate demand } z_{t}^{(k)} \text { converges to some non-zero value; } \\ \boldsymbol{\bullet}\left[\frac{\boldsymbol{r}_{t+1}+\mathbf{1}_{K}}{1+\boldsymbol{z}_{t}^{\prime}\left(\boldsymbol{r}_{t+1}+\boldsymbol{e}_{t+1}\right)}\right]_{k} \rightarrow c \in(0,1) \text { and the aggregate demand } z_{t}^{(k)} \text { converges to zero; } \\ \boldsymbol{\bullet}\left[\frac{\boldsymbol{r}_{t+1}+\mathbf{1}_{K}}{1+\boldsymbol{z}_{t}^{\prime}\left(\boldsymbol{r}_{t+1}+\boldsymbol{e}_{t+1}\right)}\right]_{k} \rightarrow c>1 \text { and the aggregate demand } z_{t}^{(k)} \text { diverges: the system is degenerate. }\end{array}\right.$

Note that in the case of nonzero aggregate demands, all returns are equal, which is consistent with the first case above. This is another illustration of the relationship between equilibrium returns and aggregate demands. When returns are inhomogeneous, the above classification shows that zero aggregate demands become likely (the other option being degeneracy of the system). We underline that this also depends on the number of agents because the number of assets with zero aggregate demands must be strictly smaller than the number of agents (see Section 3.3 for further details).

\subsection{Survival with nonzero demands and constant dividend yields}

In this section, we are interested in the dynamic behavior of the market shares $\phi_{t, n}$ when they converge to the equilibrium in the case of the deterministic skeleton. We say that an agent survives upon equilibrium if its market share is strictly positive. The purpose of this section is to identify a condition pertaining to portfolio compositions that implies survival. To this end, we introduce the characteristic ratios

$$
R_{n}=\frac{\sum_{k=1}^{K} x_{n}^{(k)} e^{(k)}}{1-\sum_{k=1}^{K} x_{n}^{(k)}}, \text { in vector form, } \boldsymbol{R}=\left(\boldsymbol{I}_{N}-\operatorname{diag}\left(\boldsymbol{x}^{\prime} \mathbf{1}_{\boldsymbol{K}}\right)\right)^{-1} \boldsymbol{x}^{\prime} \overline{\boldsymbol{e}}=\operatorname{diag}\left(\mathbf{1}_{N}-\boldsymbol{x}^{\prime} \mathbf{1}_{\boldsymbol{K}}\right)^{-1} \boldsymbol{x}^{\prime} \overline{\boldsymbol{e}}
$$

and the key matrix

$$
\boldsymbol{M}=\operatorname{diag}\left(\boldsymbol{x} \phi_{t}\right)^{-1} \boldsymbol{x} \operatorname{diag}\left(\boldsymbol{\phi}_{t}\right) \boldsymbol{x}^{\prime} .
$$

We omit the time-dependence of $\boldsymbol{M}$ for notational simplicity. Below, we detail our major results on survival.

Proposition 9. We assume condition (6) is fulfilled so that all demands are nonzero. The evolution of market shares satisfies

$$
\frac{\phi_{t+1, i}}{\phi_{t, i}}=\left(1+\boldsymbol{\phi}_{t}^{\prime} \boldsymbol{\rho}_{t+1}\right)^{-1}\left(1+\sum_{j=1}^{K} \sum_{k=1}^{K} x_{i}^{(j)} \sum_{q=0}^{\infty}\left[\boldsymbol{M}^{q}\right]_{j k} e_{t+1}^{(k)}\right) .
$$

In the case of the deterministic skeleton, if $K \geq N$, then surviving agents at a PCE all have the same $R_{n}$ equal to $r^{*}$.

\footnotetext{
${ }^{9}$ It seems intuitive that an asset with positive (resp. negative) demand be linked to future positive (resp. negative) total returns: agents ask for profits and avoid losses and prices should reflect this basic economic principle. Hence, the demand-weighted sum of future total returns should always be positive.
} 
With the above properties in hand, we are able to prove a deeper result in the case $K \geq N$. In this case, and because we are solely interested in survival, we can assume without loss of generality that all characteristic ratios are unique (or simply that the largest one is unique). The following theorem shows that having the maximum ratio is key to survival.

Theorem 10. Under (6) and when $N \leq K$, if $R_{1}$ is the maximum characteristic ratio, then the market share of the first agent $\left(\phi_{t, 1}\right)$ is the only one for which 1 is a Lyapunov stable equilibrium point for the deterministic skeleton. Formally, $\forall \epsilon>0$, there exists $\delta(\epsilon)>0$ such that if $\phi_{t, 1} \geq$ $1-\delta(\epsilon)$, then $\phi_{t+s, 1} \geq 1-\epsilon$ for all $s \geq 1$.

One way to paraphrase this result is to say that having the maximal characteristic ratio guarantees survival. When agents have fixed strategies it is always possible to compute their characteristic ratios and to determine those who survive. Note that when leverages are unconstrained, the ratios can be arbitrarily high and, in turn, the notion of optimal policy can only be defined among a given set of strategies.

When leverages are all equal, the ratios are proportional to $\boldsymbol{x}^{\prime} \overline{\boldsymbol{e}}$. Hence, the maximum ratio is reached by investing everything into the asset that pays the largest dividend yields. Unfortunately, this leads to an asymptotic violation of (6) because most aggregate demands decrease to zero. However, if we apply item $M_{37}$ of Berman and Plemmons (1994) to the inverse in (7), it is possible to show that the system in this case stays well-behaved: shares remain positive and bounded by one. Unfortunately, the asymptotic zero demands imply that the statement of Theorem 10 does not hold. This configuration in demands is nonetheless instructive and we provide an enlightening example in Appendix J.

When agents have identical leverages and their portfolios meet condition (6), the best family of strategies consist in investing a large proportion in the asset that pays the largest dividend yields and an arbitrarily small (but strictly positive) proportion to all other assets. We emphasize that there is some empirical evidence that firms with higher dividend yields are more profitable for investors (see e.g. McQueen et al. (1997) and Visscher and Filbeck (2003)).

Lastly, we comment on the condition $K \geq N$ in the theorem. Formally, we were only able to prove the result when the number of agents is no larger than that of assets. Empirically, thousands of simulations with various portfolio configurations have shown that the maximum $R_{n}$ policy always dominates the market. While we have not been able to prove the stability criterion in the general case, it is possible to show the following implication: $\boldsymbol{r}_{t}=r^{*} \mathbf{1}_{K} \Rightarrow \phi_{t+1, k} \geq \phi_{t, k}$ when $k$ corresponds to the agent with maximal characteristic ratio. When uncertainty has faded and returns have converged, having the highest characteristic ratio warrants a non-decreasing market share. We formally state and provide the proof of this result in Appendix K.

\subsection{Random dividend yields}

A natural question that arises pertains to the validity of the above result when the dividends are no longer constant. The usual argument is that if the randomness is modest, then the system is only marginally perturbed and converges to the fixed-state described in the deterministic case. In different contexts, Benaïm and Weibull (2003) and Zhu et al. (2011b) show that stochastic systems asymptotically converge to the deterministic equilibria when the noise is sufficiently small. Below, we formally discuss this assertion for our model in more detail.

We seek to characterize the condition under which the survival result in Theorem 10 remains true. Starting from the vector of average dividends $\bar{e}$, we add some noise in the following fashion: time-varying dividends are constructed as perturbations around their average values: $e_{t+1}^{(k)}=\bar{e}^{(k)}+$ $\xi_{t+1}^{(k)}$, where $\xi^{(k)}=\left\{\xi_{t}^{(k)}, t \geq 0\right\}$ is a real valued stochastic process in discrete time defined on 
$\left(\Omega,\left(\mathcal{F}_{t}\right)_{t \in \mathbb{N}}, \mathbb{P}\right)$. The noise process $\xi^{(k)}$ has a zero mean and support $S_{k}=\left(-\kappa^{(k)}, \kappa^{(k)}\right)$, so that dividends have support

$$
I_{k}=\left(\bar{e}^{(k)}-\kappa^{(k)}, \bar{e}^{(k)}+\kappa^{(k)}\right) .
$$

Note that the positiveness of dividends imposes $\bar{e}^{(k)} \geq \kappa^{(k)}$. In the proposition below, we provide an upper bound on $\kappa^{(k)}$ under which the survival criterion of Theorem 10 holds in some probabilistic sense.

Proposition 11. Under the assumptions of Theorem 10, if the supports of the dividends are such that

$$
\max _{k} \kappa^{(k)}<\min _{n \neq 1} \frac{R_{1}-R_{n}}{\left(\frac{\lambda_{1}}{1-\lambda_{1}}+\frac{\lambda_{n}}{1-\lambda_{n}}\right)},
$$

then the Lyapunov stability holds in the following almost-sure sense: $\forall \epsilon>0, \exists \delta(\epsilon)>0$ such that if $\phi_{t, 1} \geq 1-\delta(\epsilon)$, then $\mathbb{P}\left(\left\{\phi_{t+s, 1} \geq 1-\epsilon, \forall s \geq 1\right\}\right)=1$.

Said differently, with the condition on the noise provided in Proposition 11, the result of Theorem 10 holds in a $\mathbb{P}$-almost sure sense. A notable feature of the proposition is that it imposes no structure of correlation on the dividends: neither in the cross-section dimension (the covariance matrix between assets), nor in the time domain (the auto-correlations do not matter).

The assumption that dividend yields do not vary much is in fact quite realistic. Indeed, firms know that their dividend policy is a signal sent to analysts and investors. Hence, they tend to smooth their dividends in time. Dividend contractions are viewed as an indication of poor economic health and are avoided at all cost. Symmetrically, large increases in dividends are expensive because executives know they will not be able to cut them in the future. In sum, dividends are usually quite stable. We refer to Lintner (1956), Leary and Michaely (2011) for more details on the matter.

\subsection{Links with the related literature}

In this section, we shed some light on how to interpret our results through the prism of the work of Evstigneev et al. (2008) and Sandroni (2000). ${ }^{10}$

\subsubsection{Comparison with Evstigneev et al. (2008)}

There are three major differences between the model introduced in Section 2 and that of Evstigneev et al. (2008). The first difference is that homogeneity in risky exposure (or, equivalently, in consumption) is a crucial assumption in the proofs of Evstigneev et al. (2008). In our model we do not impose such restrictions, and many simplifications do occur when we set $\boldsymbol{x}^{\prime} \mathbf{1}_{K}=\lambda \mathbf{1}_{N}$. Nevertheless, in all generality, our results underline the mixed effects stemming from the portfolio composition. We show that for a given characteristic ratio, a lower leverage can be compensated by investing more in assets with higher dividend yields. A key feature of our results is that they allow the comparison of any set of portfolio compositions, while Evstigneev et al. (2008) only focus on the optimal one and do not provide any criterion to determine which strategy will survive if none of the agents pick the best policy.

The second key discrepancy between the two approaches is that in Evstigneev et al. (2008), agents choose between risky assets and consumption while in our model, the choice is between risky assets and a riskless asset. The impact on our results of this modeling assumption is an intriguing

\footnotetext{
${ }^{10}$ We thank an anonymous referee for inviting us to dig deeper into these considerations.
} 
question. With our notation, replacing the riskless asset by consumption would imply the following law of motion for the wealths:

$$
W_{t+1, n}\left(\boldsymbol{P}_{t+1}\right)=\rho W_{t, n} \sum_{k=1}^{K} x_{t, n}^{(k)} \frac{P_{t+1}^{(k)}+D_{t+1}^{(k)}}{P_{t}^{(k)}},
$$

where $1-\rho$ is the rate of consumption, which is fixed across agents. In Appendix L, we briefly show that in all generality, this new model does indeed alter the results. In particular, equilibrium returns are no longer homogeneous across assets and, more importantly, the survival criterion does not hold anymore. Still, if we assume, as in Evstigneev et al. (2008), that $\boldsymbol{x}^{\prime} \mathbf{1}_{K}=\mathbf{1}_{N}$, then the models do share some properties. For instance, equilibrium returns in this case are uniform. In addition, the asymptotic variation of market shares when one agent dominates the economy is favorable only to the agent with maximum demand-weighted dividend yield. This condition is the same as in our model when agents have the same leverage (i.e., $\boldsymbol{x}^{\prime} \mathbf{1}_{K}=\lambda \mathbf{1}_{N}$ ). Overall, switching from the riskless asset to consumption does generate some difference between the models when demands are unconstrained. However, if agents have the same aggregate risky exposure, then the two frameworks lead to comparable behaviors and conclusions.

Lastly, the third difference of our setup with Evstigneev et al. (2008) pertains to the assumptions on dividends. In Evstigneev et al. (2008), dividends are functions of i.i.d. states of nature and they are hypothesized to fulfill some positivity conditions (individually and at the aggregate level). In the present paper, the assumptions are quite different. First of all, the individual distributions of dividend yields in our model are only determined by their support and their mean, which is assumed constant over time (see Proposition 11). Second, we impose no structure at all in the correlation of dividend yields, both across assets and across time. This is a major shift from the traditional assumptions in the literature.

Finally and most importantly, we do not work with dividends per se, but with dividend yields. Implicitly, we posit that dividend payments depend on the price level of the asset. This suggests for instance that among two firms with equal yields, the larger firm - in terms of price - will pay larger dividends. This is the main reason why the survival criteria are at odds (concentration in assets versus diversification). In Evstigneev et al. (2008), by Equation (18), the total wealth is i.i.d. and bounded from above (hence prices also have an upper limit), while in the present article, prices and wealths either increase to infinity or vanish, depending on the sign of equilibrium returns. This feature is critical because it drives the relative importance of price returns and dividend yields within total returns, which, in turn, determine who survives.

It is unfortunately impossible to harmonize both approaches because we cannot consider that both dividends and dividend yields are i.i.d.: this would imply constant prices. Moreover, the proportional betting scheme based on dividends does not have any sense in our economic setup, if only because dividends do not have constant means.

Nonetheless, there is a configuration that helps reconcile both approaches. Suppose, as in Evstigneev et al. (2008), that risky exposures are uniform across agents $\left(\boldsymbol{x}^{\prime} \mathbf{1}_{K}=\lambda \mathbf{1}_{N}\right)$ and that the first agent survives (his portfolio has maximum $\boldsymbol{x}^{\prime} \overline{\boldsymbol{e}}$ ). For simplicity and comparison purposes, we also set $r_{f}=0$ so that $\boldsymbol{P}_{t}=\boldsymbol{p}_{t}$. Then, by the first line of (4), $\boldsymbol{p}_{t} \propto \boldsymbol{x}_{1}$, asymptotically. Hence, at a PCE, $\boldsymbol{D}_{t+1}=\operatorname{diag}\left(\boldsymbol{p}_{t}\right) \boldsymbol{e}_{t+1} \propto \operatorname{diag}\left(\boldsymbol{x}_{1}\right) \boldsymbol{e}_{t+1}$. When prices have reached their stable values, the proportional dividend policy is a multiple of $\operatorname{diag}\left(\boldsymbol{x}_{1}\right) \overline{\boldsymbol{e}}$. Thus, the surviving agent in our specification and the optimal portfolio of Evstigneev et al. (2008) invest in the same assets.

\subsubsection{Connection with Sandroni (2000)}

It is possible to analyze the link between our condition based on the characteristic ratio and the rule relying on the expectation of the log-growth rate in Sandroni (2000), given in his Proposition 
1. With our notations, the latter is written for agents 1 and $n(n \neq 1)$ as: there exists $\alpha>0$ such that for all $t$ we have

$$
\mathbb{E}\left[\ln \left(1+\rho_{t+1,1}\right) \mid \mathcal{F}_{t}\right] \geq \mathbb{E}\left[\ln \left(1+\rho_{t+1, n}\right) \mid \mathcal{F}_{t}\right]+\alpha .
$$

In Sandroni (2000), this is a sufficient condition for agent $\mathrm{n}$ to vanish from the market. If we start from our rule based on the characteristic ratios, it implies a weak form of Sandroni's rule and becomes a necessary condition for agent $n$ to vanish. The derivation of this implication is given below.

We work under the assumptions of Proposition 9 and Theorem 10: we are close to equilibrium but not at equilibrium and dividend yields are stochastic. We further assume $r_{f}=0$.

First, suppose that agent 1 has maximum characteristic ratio. From the computations in the proofs of Proposition 9 and Theorem 10 we have that there is $h \geq 0$ and $\beta>0$ such that, for all $t \geq h$, given $\mathcal{F}_{t}$

$$
\frac{\phi_{t+1,1}}{\phi_{t, 1}}-\frac{\phi_{t+1, n}}{\phi_{t, n}}>\beta, \quad \mathbb{P}-\text { a.s. }
$$

and $\beta=c\left(1-\lambda_{n}\right)\left(R_{1}-R_{n}\right)$ with the notations in the above mentioned proofs. From the definition of market shares

$$
\frac{\frac{w_{t+1,1}}{\left(\mathbf{1}_{N}^{\prime} \boldsymbol{w}_{t+1}\right)}}{\frac{w_{t, 1}}{\left(\mathbf{1}_{N}^{\prime} \boldsymbol{w}_{t}\right)}}-\frac{\frac{w_{t+1, n}}{\left(\mathbf{1}_{N}^{\prime} \boldsymbol{w}_{t+1}\right)}}{\frac{w_{t, n}}{\left(\mathbf{1}_{N}^{\prime} \boldsymbol{w}_{t}\right)}}>\beta, \quad \mathbb{P}-\text { a.s. }
$$

that is

$$
\rho_{t+1,1}-\rho_{t+1, n}>\beta \frac{\mathbf{1}_{N}^{\prime} \boldsymbol{w}_{t+1}}{\mathbf{1}_{N}^{\prime} \boldsymbol{w}_{t}}, \quad \mathbb{P}-\text { a.s. }
$$

And we finally get, because $\frac{\mathbf{1}_{N}^{\prime} \boldsymbol{w}_{t+1}}{\mathbf{1}_{N}^{\prime} \boldsymbol{w}_{t}}>0$ but not greater than a constant independent of $t$

$$
\rho_{t+1,1}-\rho_{t+1, n}>0, \quad \mathbb{P}-\text { a.s. }
$$

We also have

$$
\ln \left(1+\rho_{t+1,1}\right)>\ln \left(1+\rho_{t+1, n}\right), \quad \mathbb{P}-\text { a.s. }
$$

which implies

$$
\mathbb{E}\left[\ln \left(1+\rho_{t+1,1}\right) \mid \mathcal{F}_{t}\right] \geq \mathbb{E}\left[\ln \left(1+\rho_{t+1, n}\right) \mid \mathcal{F}_{t}\right] .
$$

This is a weak form of the condition in Sandroni (2000). Under the setup described above, the rule we obtain in our market model implies a weak form of the rule devised in Proposition 1 of Sandroni (2000). Hence, where Sandroni's Proposition 1 gives a sufficient condition for an agent to vanish in terms of log-growth rates, our rule implies a related necessary condition for an agent to vanish.

\subsubsection{General remarks}

At a deeper level, we shortly comment on the way our reasoning differs from that of existing contributions. Devising an optimal fixed strategy requires stability in the modeling assumptions. In the bulk of the literature, this stability stems from i.i.d. randomness that drives wealth or perturbs the system. This is for instance the path taken by the literature on Kelly schemes, initiated in Kelly (1956) and thoroughly surveyed in MacLean et al. (2011). Both Breiman (1960) and Finkelstein and Whitley (1981) assume i.i.d. payoffs and are able to single out an optimal fixed strategy. When noise is no longer i.i.d., as in Algoet and Cover (1988) and Sandroni (2000), then optimal portfolio decisions must be time-dependent. 
Proposition 1 of Sandroni (2000) provides a sufficient condition that ensures survival for one agent: conditionally on prior information, this agent must have strictly superior expected logarithmic (gross) returns at all times, compared to all other agents. This is an intuitive result but it is too general since the condition is very binding. Proposition 2 and Proposition 4 in the same paper are much more specific and insightful: assuming homogeneous discount factors across agents, it is shown that agents who survive are those who eventually make accurate predictions. It is the end of the trajectory that matters and not its beginning. In spirit, this reasoning is very close to the way we proceed. Since total returns are unknown before equilibrium in our model, survival cannot be determined in advance when portfolios are fixed. As is shown in Appendix $\mathrm{K}$, the only agents who are assured to have growing market shares at the PCE are those with maximal characteristic ratios. In a sense, they are those who are eventually able to make the most accurate predictions. This is why the concept of PCE is so useful. Working on fixed points allows to introduce stability in payoffs and hence to determine which fixed portfolios are asymptotically optimal.

\section{Numerical investigations}

\subsection{Nonzero demands}

We illustrate the survival criterion of Theorem (10) with a simple example. The portfolio compositions, dividend yields and initial market shares are defined in Table 1. We consider two cases. In the first one, Agent 1 and Agent 2 have the same characteristic ratio, but in the second one, Agent 1 has the highest ratio of all. The first two agents begin with a $30 \%$ market share, with the remaining $40 \%$ going to the third agent.

\begin{tabular}{|c|c|c|c|c|c|c|c|c|c|c|}
\hline & \multicolumn{5}{|c|}{ Case 1} & \multicolumn{5}{|c|}{ Case 2} \\
\hline & Agent 1 & Agent 2 & Agent 3 & $\bar{e}$ & TAD & Agent 1 & Agent 2 & Agent 3 & $\bar{e}$ & TAD \\
\hline Asset 1 & 0.200 & 0.100 & 0.050 & 0.010 & 0.149 & 0.200 & 0.000 & 0.050 & 0.010 & 0.183 \\
\hline Asset 2 & 0.200 & 0.120 & 0.100 & 0.020 & 0.159 & 0.200 & 0.120 & 0.100 & 0.020 & 0.193 \\
\hline Asset 3 & 0.200 & 0.100 & 0.000 & 0.030 & 0.149 & 0.200 & 0.100 & 0.000 & 0.030 & 0.192 \\
\hline Asset 4 & 0.030 & 0.300 & 0.000 & 0.022 & 0.168 & 0.030 & 0.300 & 0.000 & 0.022 & 0.053 \\
\hline$R_{n}$ & 0.034 & 0.034 & 0.003 & - & - & 0.034 & 0.025 & 0.003 & - & - \\
\hline
\end{tabular}

Table 1: Parametrization. We provide agents' demands $\left(x_{n}^{(k)}\right)$ along with asset dividend yields $(\overline{\boldsymbol{e}})$ and corresponding $R_{n}$. Initial returns are equal to zero for all assets. TAD stands for terminal aggregate demand. In the first case, the first two agents have equal $R_{n}$, and in the second case, all $R_{n}$ differ. The discrepancies between the two cases are highlighted in bold. Condition (6) is satisfied.

In Figure K.9, we plot the time-series of market shares $\left(\phi_{t, n}\right)$ and price returns $r_{t}^{(k)}$ for case 1 (left) and case 2 (right). In the bottom graphs, we see that the convergence to the (unique) equilibrium return of Proposition 4 is rather slow. After 300 (left) and 500 (right) iterations of the system, the fourth asset's return is still a few percentage points away from the target value. Numerical simulations of the system as well as plots presented throughout Section 6 are done with $\mathrm{R}$, version 3.4.3. The code is available upon request.

In the upper plots of Figure K.9, we observe an illustration of Proposition 9. In the left graph, Agent 1 and Agent 2 have equal characteristic ratios, and hence, their market share does not vanish, but rather increases to some terminal value (close to $50 \%$ ). On the contrary, the weight of Agent 3, who has a lower ratio, decreases to zero. In the right graph, only one agent survives: his market share progressively increases to one. Agent 1's higher characteristic ratio translates into a dominating position. Note that the very small change in parameters between the two cases does not alter the final values of returns. 
Case 1
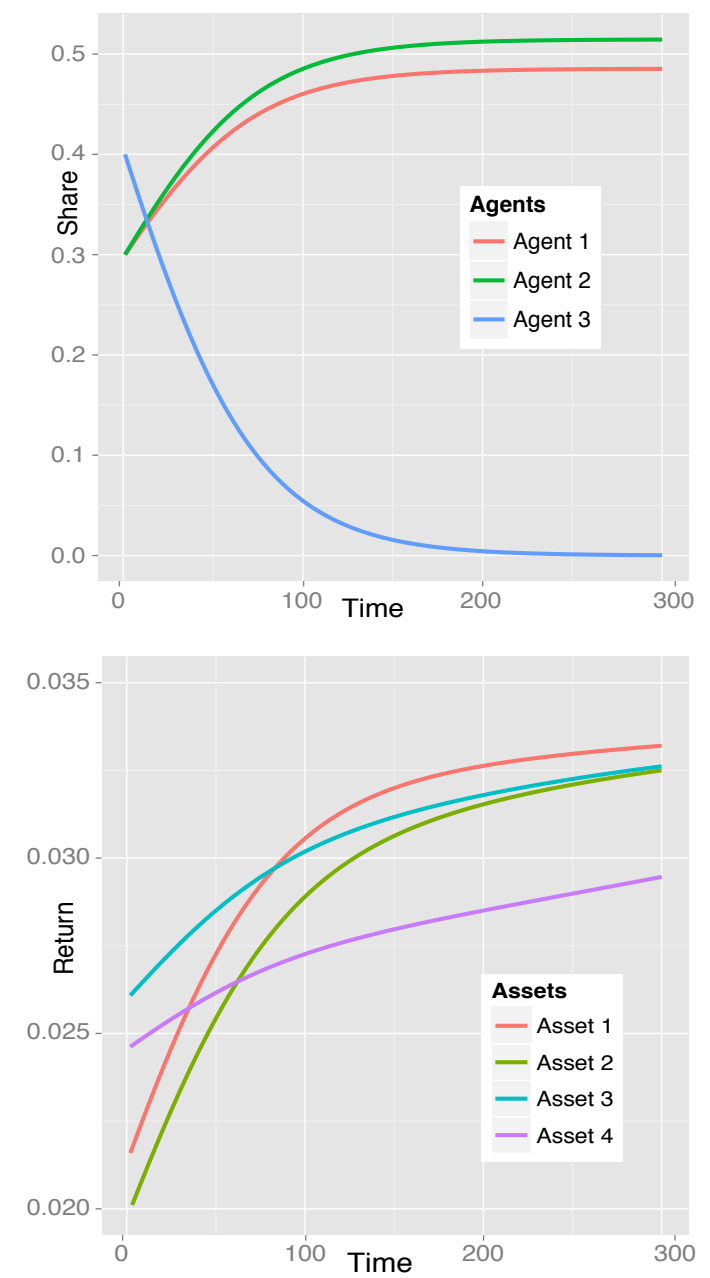

Case 2
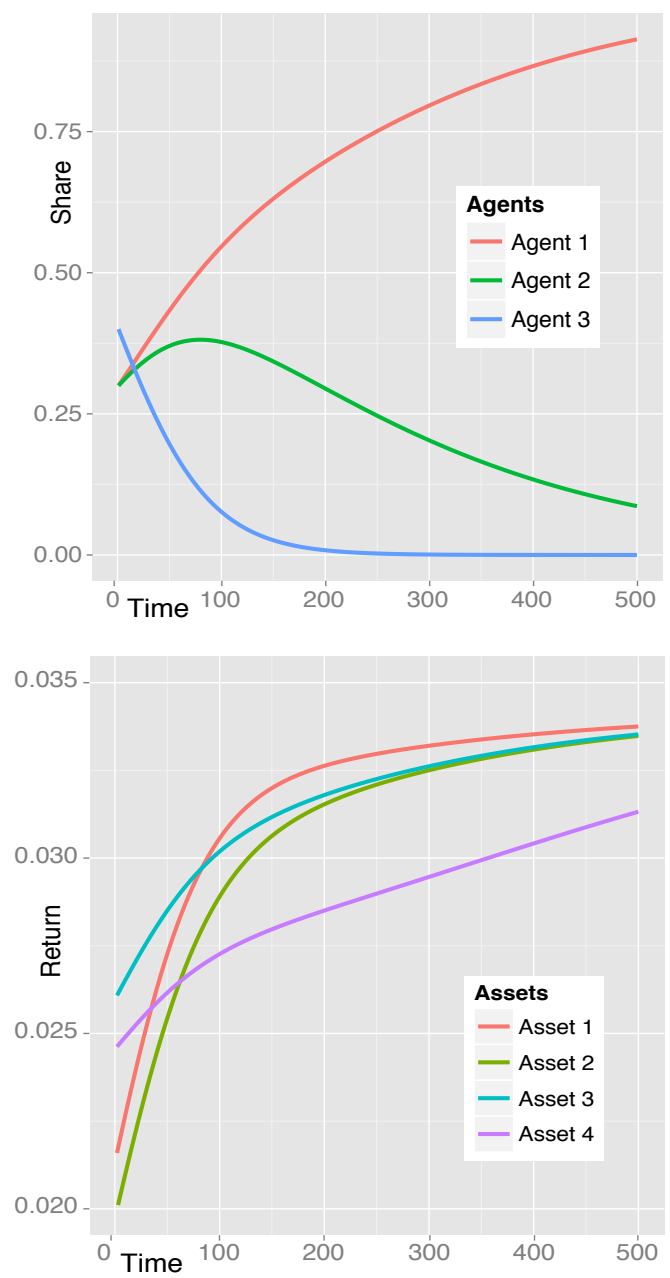

Figure 2: Non-zero demands. We plot agent shares (top) and asset returns (bottom) with case 1 (and maturity $T=300$ ) on the left and case 2 (with $T=500$ ) on the right. The equilibrium return in both cases is given by (14) is equal to 0.0342 .

\subsection{Zero demands}

Obviously, assets in financial markets do not have equal price returns. We thus propose a new configuration which will lead to only one asset with nonzero demand. As is recalled in Proposition 5 , this requires more agents than assets with zero demands. We thus stick with four assets, but now consider five agents (one of whom will vanish). Agents start with uniform market shares. In order to reach zero demands, all agents resort to short-selling for at least one asset. The details of the parametrization are given in Table 2. We underline that not all configurations lead to interesting interpretations. As is suggested by Equation (32), some even imply degenerate skeletons with negative market shares and/or infinite returns.

In Figure 3, we plot the time-series of aggregate demands (left) and asset total returns (right). In addition, we provide the static proxy for the returns provided by equation (23). Obviously, the values are expected to coincide at equilibrium, and the plot allows to monitor the speed of this convergence. In Figure 4, we plot agent shares. The left graph corresponds to the case when they have initial equal weight while in the right graph, we have set $\phi_{0}=(0.15,0.05,0.1,0.1,0.6)$.

Given the dynamical essence of the system, we take a chronological approach in our analysis:

1. We first see on the right graph that assets with negative aggregate demand (1 and 3 ) start by 


\begin{tabular}{lrrrrr|rr} 
& Agent 1 & Agent 2 & Agent 3 & Agent 4 & Agent 5 & $\overline{\boldsymbol{e}}$ & Raw demand \\
\cline { 2 - 7 } Asset 1 & 0.020 & 0.400 & -1.015 & -0.190 & -0.150 & 0.010 & -0.935 \\
Asset 2 & -0.200 & 1.120 & 0.090 & 0.130 & -0.180 & 0.020 & 0.960 \\
Asset 3 & 0.200 & 0.000 & -0.200 & -1.250 & 0.800 & 0.030 & -0.450 \\
Asset 4 & 1.090 & -0.300 & 0.294 & 0.060 & 0.600 & 0.022 & 1.744 \\
Exposition & 1.110 & 1.220 & -0.831 & -1.250 & 1.070 & - & - \\
Leverage & 1.510 & 1.820 & 1.599 & 1.630 & 1.730 & - & - \\
$R_{n}$ & -0.238 & -0.090 & -0.004 & -0.016 & -0.459 & - & - \\
$\phi_{0}$ & 0.200 & 0.200 & 0.200 & 0.200 & 0.200 & - & - \\
\hline
\end{tabular}

Table 2: Parametrization. Agents' demands $\left(x_{n}^{(k)}\right)$ are shown along with asset dividend yields $(\overline{\boldsymbol{e}})$ and corresponding $R_{n}$. Original market shares $\phi_{0}$ are provided on the last line. Initial returns are equal to zero for all assets. Exposition is computed as the sum of an agents positions $\sum_{k=1}^{K} x_{n}^{(k)}$. Leverage is the sum of positions in absolute value. Raw demand is the aggregate demand $\boldsymbol{x} \phi$ when the distribution of agents is uniform: $\boldsymbol{\phi}=\mathbf{1}_{N} / N$. The terminal aggregate demands are all null, except for the second asset which has a TAD of 0.703 . The agents' demands do not satisfy condition (6).
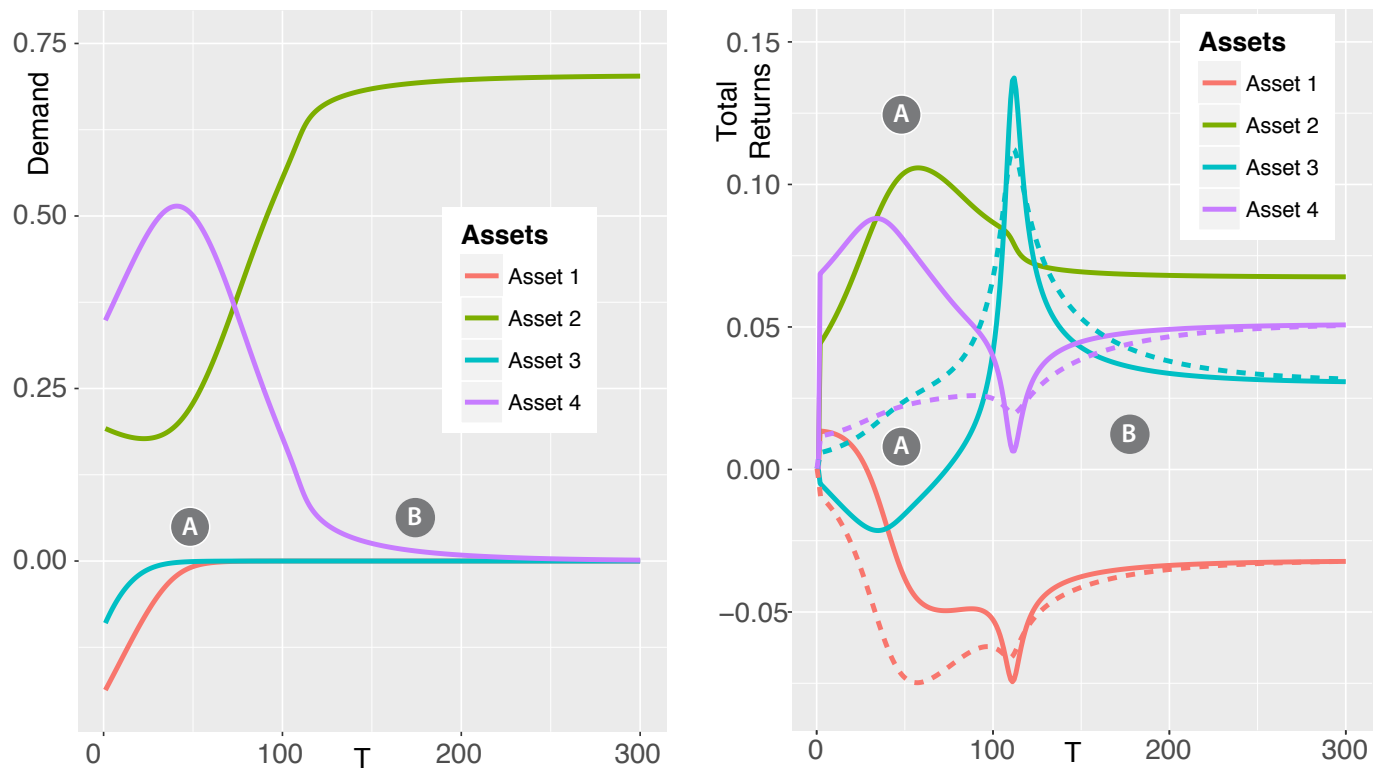

Figure 3: Zero demands. We plot the evolution of aggregate demands (left) and asset total returns (right) related to the deterministic skeleton with parameters of Table 2. In the right graph, the dotted lines show the proxy for total returns of ZD assets computed using the equilibrium relationship of Equation (23).

having negative returns, while it is logically the opposite for the other two (assets 2 and 4). The total return structure is also favorable to these two assets. Mechanically, the agents who have the largest cumulative positions in these assets see their market share increase (Agents 1 and 2).

2. Then, we arrive around point $\mathrm{A}(t \approx 50)$ in the Figures. At this point, the demands for assets 1 and 3 are already very close to zero and will remain negligible as shown by Lemma 8. Consequently, the returns of assets 1 and 3 are not driven by their demand but by other quantities. We can thus check the empirical relevance of equation (23) at this point. The dotted curves in Figure 3 show the theoretical equilibrium relationships. The spreads with the true values are still sizable at point $\mathrm{A}$, but the trends seem preserved. Indeed, after 

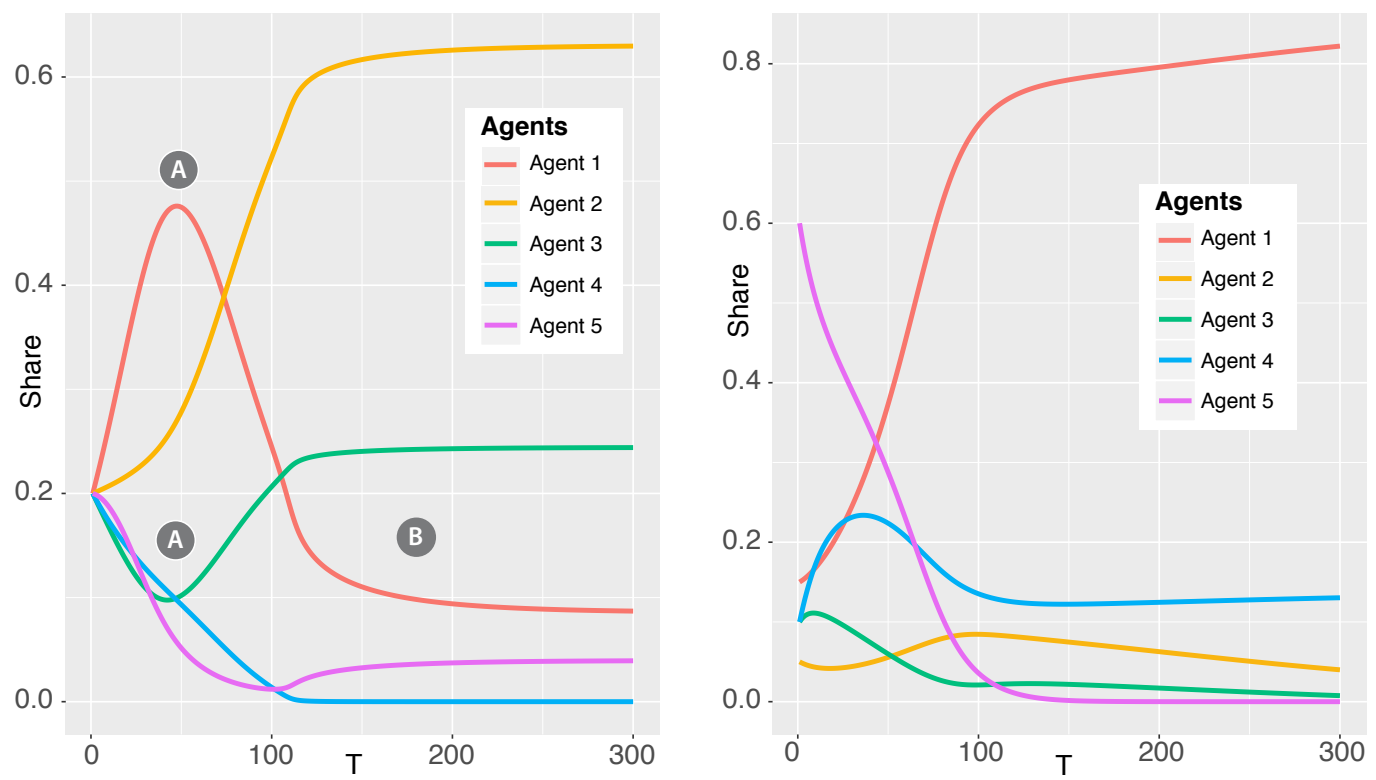

Figure 4: Market shares under some zero demands. We plot agent market shares with parameters of Table 2 and $\phi_{0}=(0.2,0.2,0.2,0.2,0.2)$ on the left and $\phi_{0}=(0.15,0.05,0.1,0.1,0.6)$ on the right.

point A, the total return of asset 3 is starting to increase, and follows the tendency of the dotted turquoise line. Likewise, for asset 1, even though a small positive bump occurs, the total returns are bound to stay significantly negative. As for asset 4 , the true value clearly does not mimic the proxy, simply because the aggregate demand for this asset is still largely positive.

3. Progressively, we reach point $\mathrm{B}$, just before $t=200$, when the demand for asset 4 vanishes. After this point, the hard and dotted curves start to coincide and all indicators and quantities have approximately reached their equilibrium values.

Finally, the comparison of the two graphs in Figure 4 show how initial market shares impact the final outcome. In the first case, only agent 4 vanishes, while in the second one, agents 3 and 5 both disappear. The best strategies also differ: agent 2 dominates when initial weights are equal, but agent 1 prevails in the second configuration. An classical intuition is that dominating positions are meant to persist because superior financial power can either influence the market or at least reap benefits and dividends over the long run. The right graph of Figure 4 shows that ill-advised strategies are particularly hurtful. While agent 5 starts with more than half of the market shares, its portfolio composition appears to be (by far) the least profitable (or the most penalizing) because it leads to an irreversible decay.

\subsection{Random dividends}

In this final subsection, we add noise to the deterministic trajectories studied above. In line with the notations of Section 5.3, the support of the dividends is symmetric around their mean: $I_{k}=\left[\bar{e}^{(k)}-\kappa^{(k)}, \bar{e}^{(k)}+\kappa^{(k)}\right]$. We work with four values for $\kappa^{(k)}: 0.001,0.005,0.01$ and $\bar{e}^{(k)}$. The first three values are fixed, meaning that the dispersion (standard deviation) is equal across assets. The fourth alternative, corresponds to the highest level of dispersion compatible with the positivity of dividends. This limiting case implies that higher dividends on average are more volatile: the standard deviation is proportional to the mean. Thus, in the figures below, this case is referred to 
as 'Prop.. Here we have implicitly assumed, for simplicity, that the support is symmetric around the mean, as we did in Section 5.3.

For each one of the four values of amplitudes $\kappa^{(k)}$ detailed above, we run 10,000 simulations of the system up to time $T=500$. This is a reasonable horizon for the convergence of the system. Henceforth, 'terminal' values are associated to this maturity. Dividends are sampled uniformly on their support and are independent both in time and in the cross-section. In Figures 5 and 6 , we illustrate the configurations with non-zero demands of Section 6.1. Each Figure pertains to a different type of agent: Agent 1 (in Figure 5) survives, while Agent 3 (in Figure 6), vanishes.

In the top graphs of Figure 5, we show the distribution of Agent 1's terminal market share. Both distributions are centered around the value reached in the deterministic case $(0.485$ in Case 1 and 0.913 in Case 2). Additionally, the distributions confirm the intuition: as the amplitude of the support of the dividends increases, then so does the dispersion of the market shares. Nonetheless, even when dividends are allowed to vary between zero and twice their average value (purple bars), the histograms clearly indicate that Agent 1 will survive.

This is further confirmed by the lower graphs which plot the evolution of the market shares through time. In the right graph, survival is unambiguous and Agent 1 eventually fully dominates the market. Just as in the deterministic case, this was expected because Agent 1 has the highest characteristic ratio. We see that all curves display a similar behavior, even when the shocks are the largest. This corroborates the theoretical Proposition $11 .{ }^{11}$ If the left graph seems less compelling, the conclusion remains the same: Agent 1 will ultimately survive as well. In this case, the market share of Agent 3 rapidly decreases to zero and the Brownian-like variations of Agent 1's market shares only reflect the economic confrontation with Agent 2. These two agents have their shares updated by the current levels of dividends but none of them can asymptotically beat the other because they have the same characteristic ratio.

In order to illustrate the situation of a vanishing agent, we plot the same quantities for Agent 3 in Case 1 in Figure 6. The distribution of market shares is highly concentrated around a very small figure $\left(1.7 \times 10^{-6}\right)$ and even when dividends have the largest dispersion, the final market share does not exceed $4 \times 10^{-6}$. A look at the trajectories confirms the ineluctable disappearance of the agent. We show the path between $t=100$ and $t=300$ so that the differences between amplitudes are discernible. Indeed, in contrast with Agent 1's market share in Case 1, there is not much variety in the behavior of Agent 3's relative wealth: it inevitably shrinks to zero at a pace that does not depend on the dispersion of dividends. We do not report the evolution of market share for Agent 3 in Case 2 because the numerical results are almost identical to those plotted for Case 1 in Figure 6.

We then switch to the case with asymptotic zero aggregate demands. As is underlined in Section 5.1, in such configurations, the system may reach a singularity and lead, for instance, to negative market shares. Among the 10,000 simulations we ran, some of them did yield degenerate trajectories. The proportion of these paths varies strongly with the size of the support of the dividends. For the three fixed values of $\kappa^{(k)}$, it is equal to $0,0.1 \%$ and $9.4 \%$, respectively. When $\kappa^{(k)}=\bar{e}^{(k)}$, the proportion increases to $39.6 \%$.

\footnotetext{
${ }^{11}$ In fact, the upper bound in the Proposition is far from optimal and rather conservative when dividends are jointly i.i.d. With the parametrization of Case 2, this bound is equal to 0.0033 . Hence, only the first amplitude fulfills the condition but we see that survival is ensured even with larger values of $\kappa^{(k)}$. This is because in the proof of the Proposition, we consider the least favorable scenario at all times: lowest possible dividends for Agent 1 and highest possible dividends for other agents: it is the price to be paid for almost sure convergence in the general case with no particular assumption on correlations. Hence, the upper bound on $\kappa^{(k)}$ can probably be sharpened, i.e., increased, in the case of serially and cross-sectionally uncorrelated dividends.
} 



Figure 5: Market shares with non-zero demands - Agent 1. The upper graphs show the distribution of terminal $(T=500)$ share for the first agent. The histograms are stacked and the colors relate to the amplitude $\left(\kappa^{(k)}\right)$ of the support around the means. The lower graphs plot the first ten trajectories of Agent 1's market share. Each column (left and right) corresponds to one parametrization in Table 1.

In this last case, we study the distribution of the time at which degeneracy occurs. ${ }^{12}$ To do so we denote by $\tau$ the first date when a market share reaches a negative value:

$$
\tau=\inf \left\{t \geq 0 ; \exists n \text { s.t. } \phi_{t, n}<0\right\}
$$

and, by convention, we set $\tau=+\infty$ if the system is well-behaved. $\tau$ is a stopping with respect to the filtration $\mathcal{F}$. Figure 7 represents the histogram of values smaller than 500 taken by $\tau$ over

${ }^{12}$ We thank an anonymous referee for pointing this question to our attention. 



Figure 6: Market shares with non-zero demands - Agent 3. The left graph shows the distribution of the terminal $(T=500)$ share of the third agent. The histograms are stacked and the colors relate to the amplitude $\left(\kappa^{(k)}\right)$ of the support around the means. The right graph plots the first ten trajectories of Agent 3's market share. Both graphs correspond to the first parametrization in Table 1 (Case 1).

the 10,000 trajectories. This figure allows us to visualize the distribution of $\tau$ conditional on the appearance of a degeneracy before $T=500$. We report that $95 \%$ of the negative values are attained between $t=100$ and $t=200$ and none after $t=350$. From this, we infer that $T=500$ is a suitable horizon to have a good description of the long run dynamics of the system, in particular if it is stable or explosive. This numerical result is a further confirmation that it makes sense to put the deterministic skeleton at the center of our formal study.



Figure 7: First occurrence of degeneracy. We plot the distribution of the first time that a market shares falls strictly below zero, conditionally on the fact that it happens before $T=500$ (i.e., well-behaved trajectories are discarded).

The case with large uncertainty in dividends is the most interesting one and we further investigate its properties. In Figure 8, we plot the evolution of market shares and aggregate demands for the first ten well-behaved simulations we ran with $\kappa^{(k)}=\bar{e}^{(k)}$. We do observe randomness in the paths, but all converge fairly rapidly and around $t=150$, all points are highly concentrated and clearly correspond to the deterministic trajectory. The convergence is obviously faster for smaller 
values of $\kappa^{(k)}$. This is a final illustration that the deterministic skeleton is a good approximation of the stochastic system, as long as the perturbations are sufficiently small - provided that the trajectory is not degenerate.
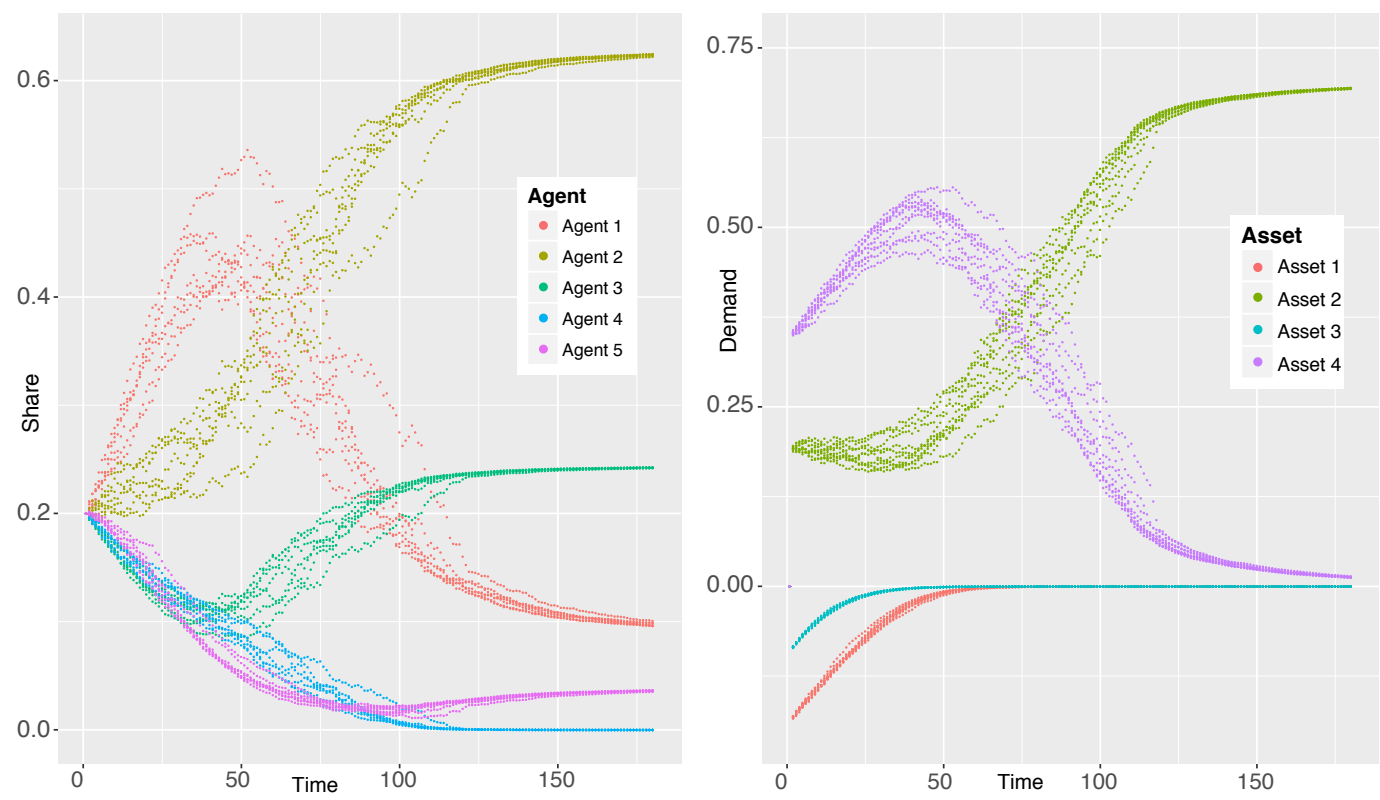

Figure 8: Zero demands. The left graph plots the market shares of Agents under the parameter values described in Table 2. The right graph shows the corresponding aggregate demands for assets.

In order to gauge the likelihood of facing such a degeneracy of the system, we simulated a large number of trajectories for fixed values of $\kappa^{(k)}$, namely $0.1 \%, 0.5 \%$ and $1 \%$, as well as $\kappa^{(k)}=\bar{e}^{(k)}$. For each set of parameters we ran a total of one million simulations organized as 100 batches of 10,000 trajectories of 500 time steps. This large scale simulation study encompasses two billion instances of our market model. For each batch we computed the probability of facing a degenerate system. Having 100 such batches allows us to quantify the variability of our estimation. We compile the descriptive statistics of our results in Table 3. Again, the risk of degenerative systems is in line with the intuition: the probability that a trajectory leads to a degenerate system increases with the magnitude of the noise. Namely, when the maximum noise is $0.1 \%$ or $0.5 \%$ we almost never face a degenerate system. However, when the noise is qualitatively large, the risk is fairly high (around $40 \%)$.

\begin{tabular}{lcccc} 
& $\kappa^{(k)}=0.1 \%$ & $\kappa^{(k)}=0.5 \%$ & $\kappa^{(k)}=1 \%$ & $\kappa^{(k)}=\bar{e}^{(k)}$ \\
\hline Minimum & 0.0000 & 0.0000 & 0.0775 & 0.3690 \\
1st Quartile & 0.0000 & 0.0010 & 0.0865 & 0.3849 \\
Median & 0.0000 & 0.0010 & 0.0913 & 0.3930 \\
Mean & $\mathbf{0 . 0 0 0 0}$ & $\mathbf{0 . 0 0 1 3}$ & $\mathbf{0 . 0 9 1 2}$ & $\mathbf{0 . 3 9 3 0}$ \\
3rd Quartile & 0.0000 & 0.0015 & 0.0956 & 0.4011 \\
Maximum & 0.0000 & 0.0035 & 0.1035 & 0.4165
\end{tabular}

Table 3: Simulation results. Distribution of the probability of facing a degenerate system when simulating trajectories for different levels of the noise added to the dividend processes. To obtain these results we ran 100 batches of 10,000 trajectories of 500 time steps. The mean (bold) corresponds to the unbiased estimation, using one million simulations, of the proportion of trajectories leading to a degenerate system. 
Overall, the numerical investigations carried out in this section confirm the formal results obtained above and they also allow us to conjecture that Proposition 11 can be extended to the case with some zero demands, as long as the trajectories are well-behaved.

\section{Conclusion}

The generalization of the framework of Anufriev and Bottazzi (2010) to many assets yields a rather tractable model for asset returns. When all aggregate demands are non null, equilibrium returns are all equal and it is possible to determine a criterion that ensures survival upon equilibrium. This criterion holds in an almost sure sense when dividends are allowed to oscillate closely to their means. When some aggregate demands vanish, the relationship between returns, demands and dividend yields is altered. In this case, the market-weighted matrix of cross-demands plays a determinant role.

Unfortunately, zero aggregate demands are much harder to handle analytically. First, they can lead to a degenerate model, with negative or infinite market shares for instance, and the conditions that ensure the stability of the model in this case are still to be determined in closed form. Second, the diversity in returns at an equilibrium point complicate the task of finding "winning" strategies ex-ante. Our intuition is that initial market shares are likely to shift the relative profitability of portfolio policies. Future work will be required to theoretically or empirically confirm or contradict this conjecture and also to further assess the properties of models when agents can increase their leverage through short-selling.

\section{Acknowledgments}

The authors gratefully thank two anonymous referees for their numerous constructive remarks.

\section{Appendix A. Proof of Proposition 1}

Plugging the third expression of the system (4) in the first, we straightforwardly get

$$
\boldsymbol{p}_{t+1}=\boldsymbol{x}_{t+1} \operatorname{diag}\left(\boldsymbol{w}_{t}\right)\left(\mathbf{1}_{N}+\boldsymbol{x}_{t}^{\prime}\left[\operatorname{diag}\left(\boldsymbol{p}_{t}\right)^{-1} \boldsymbol{p}_{t+1}-\mathbf{1}_{K}+\boldsymbol{e}_{t+1}\right]\right) .
$$

Then, isolating the terms in $\boldsymbol{p}_{t+1}$, we obtain

$$
\begin{aligned}
\boldsymbol{p}_{t+1} & =\left(\boldsymbol{I}_{K}-\boldsymbol{x}_{t+1} \operatorname{diag}\left(\boldsymbol{w}_{t}\right) \boldsymbol{x}_{t}^{\prime} \operatorname{diag}\left(\boldsymbol{p}_{t}\right)^{-1}\right)^{-1}\left(\boldsymbol{x}_{t+1} \operatorname{diag}\left(\boldsymbol{w}_{t}\right)\left[\mathbf{1}_{N}+\boldsymbol{x}_{t}^{\prime}\left(\boldsymbol{e}_{t+1}-\mathbf{1}_{K}\right)\right]\right) \\
& =\operatorname{diag}\left(\boldsymbol{p}_{t}\right)\left(\operatorname{diag}\left(\boldsymbol{x}_{t} \boldsymbol{w}_{t}\right)-\boldsymbol{x}_{t+1} \operatorname{diag}\left(\boldsymbol{w}_{t}\right) \boldsymbol{x}_{t}^{\prime}\right)^{-1}\left(\boldsymbol{x}_{t+1} \operatorname{diag}\left(\boldsymbol{w}_{t}\right)\left[\mathbf{1}_{N}+\boldsymbol{x}_{t}^{\prime}\left(\boldsymbol{e}_{t+1}-\mathbf{1}_{K}\right)\right]\right),
\end{aligned}
$$

where we have used the identity $\boldsymbol{p}_{t}=\boldsymbol{x}_{t} \boldsymbol{w}_{t}$. Equation (A.1) is the vector analogue of the expression obtained in the proof of Proposition 2.1 in Anufriev and Bottazzi (2010). The first formula of the proposition is proved and we now turn to the second statement.

We proceed iteratively and assume that $\boldsymbol{p}_{t}$ has only positive elements, so that all wealths are also positive. We factor (A.1) into three matrices: $\operatorname{diag}\left(\boldsymbol{p}_{t}\right) \boldsymbol{A}^{-1} \boldsymbol{B}$. Under $6, \boldsymbol{B}$ is strictly positive. The transposed form $\boldsymbol{A}^{\prime}=\operatorname{diag}\left(\boldsymbol{x}_{t} \boldsymbol{w}_{t}\right)-\boldsymbol{x}_{t} \operatorname{diag}\left(\boldsymbol{w}_{t}\right) \boldsymbol{x}_{t+1}^{\prime}$ implies that it is a $Z$-matrix (that is, with negative off-diagonal elements). The sum of the $k^{\text {th }}$ line of $\boldsymbol{A}^{\prime}$ is equal to

$$
\sum_{n=1}^{N} x_{t, n}^{(k)} w_{t, n}-\sum_{j=1}^{K} \sum_{n=1}^{N} x_{t, n}^{(k)} w_{t, n} x_{t+1, n}^{(j)}=\sum_{n=1}^{N} x_{t, n}^{(k)} w_{t, n}\left(1-\sum_{j=1}^{K} x_{t+1, n}^{(j)}\right),
$$


which is strictly positive when (6) holds. As such, $\boldsymbol{A}^{\prime}$ is strictly diagonally dominant and is nonsingular (see e.g. Berman and Plemmons (1994), chapter 6). This implies that $\boldsymbol{A}^{\prime}$ is in fact an $M$-matrix and its inverse has positive elements only. Therefore, since $\boldsymbol{A}^{-1}=\left(\left(\boldsymbol{A}^{\prime}\right)^{-1}\right)^{\prime}$, when the initial prices are all positive, then recursively, the prices remain always positive.

The vector of returns is given by

$$
\begin{aligned}
\boldsymbol{r}_{t+1} & =\operatorname{diag}\left(\boldsymbol{p}_{t}\right)^{-1} \boldsymbol{p}_{t+1}-\mathbf{1}_{K} \\
& =\left(\operatorname{diag}\left(\boldsymbol{p}_{t}\right)-\boldsymbol{x}_{t+1} \operatorname{diag}\left(\boldsymbol{w}_{t}\right) \boldsymbol{x}_{t}^{\prime}\right)^{-1}\left(\boldsymbol{x}_{t+1} \operatorname{diag}\left(\boldsymbol{w}_{t}\right)\left[\mathbf{1}_{N}+\boldsymbol{x}_{t}^{\prime}\left(\boldsymbol{e}_{t+1}-\mathbf{1}_{K}\right)\right]\right)-\mathbf{1}_{K} \\
& =\left(\operatorname{diag}\left(\boldsymbol{x}_{t} \boldsymbol{w}_{t}\right)-\boldsymbol{x}_{t+1} \operatorname{diag}\left(\boldsymbol{w}_{t}\right) \boldsymbol{x}_{t}^{\prime}\right)^{-1}\left(\boldsymbol{x}_{t+1} \operatorname{diag}\left(\boldsymbol{w}_{t}\right)\left[\mathbf{1}_{N}+\boldsymbol{x}_{t}^{\prime}\left(\boldsymbol{e}_{t+1}-\mathbf{1}_{K}\right)\right]\right)-\mathbf{1}_{K},
\end{aligned}
$$

where we have simply used the first line of (4) in the last equality. The second equality for $\boldsymbol{r}_{t+1}$ comes from basic calculus along with the identity (to be extensively used in most proofs):

$$
\boldsymbol{x}^{*} \operatorname{diag}\left(\boldsymbol{\phi}^{*}\right) \mathbf{1}_{N}=\operatorname{diag}\left(\boldsymbol{x}^{*} \boldsymbol{\phi}^{*}\right) \mathbf{1}_{K}=\boldsymbol{x}^{*} \boldsymbol{\phi}^{*} .
$$

Note that (A.3) is obvious given (A.1). The growth rates of wealths are easy to compute given the third line of $(4)$ :

$$
\boldsymbol{w}_{t+1}=\operatorname{diag}\left(\boldsymbol{w}_{t}\right)\left(\mathbf{1}_{N}+\boldsymbol{x}_{t}^{\prime}\left[\boldsymbol{r}_{t+1}+\boldsymbol{e}_{t+1}\right]\right),
$$

and dividing both sides by the total wealth at time $t+1$ (that is, $\mathbf{1}_{N}^{\prime} \boldsymbol{w}_{t+1}$ ), we get the dynamics of the wealth shares

$$
\boldsymbol{\phi}_{t+1}=\frac{\operatorname{diag}\left(\boldsymbol{w}_{t}\right)\left(\mathbf{1}_{N}+\boldsymbol{x}_{t}^{\prime}\left[\boldsymbol{r}_{t+1}+\boldsymbol{e}_{t+1}\right]\right)}{\mathbf{1}_{N}^{\prime} \operatorname{diag}\left(\boldsymbol{w}_{t}\right)\left(\mathbf{1}_{N}+\boldsymbol{x}_{t}^{\prime}\left[\boldsymbol{r}_{t+1}+\boldsymbol{e}_{t+1}\right]\right)} .
$$

If we divide the numerator and denominator by the total wealth at time $t$, this is equivalent to

$$
\phi_{t+1}=\operatorname{diag}\left(\phi_{t}\right) \frac{\left(\mathbf{1}_{N}+\boldsymbol{x}_{t}^{\prime}\left[\boldsymbol{r}_{t+1}+\boldsymbol{e}_{t+1}\right]\right)}{\mathbf{1}_{N}^{\prime} \operatorname{diag}\left(\boldsymbol{w}_{t}\right)\left(\mathbf{1}_{N}+\boldsymbol{x}_{t}^{\prime}\left[\boldsymbol{r}_{t+1}+\boldsymbol{e}_{t+1}\right]\right) /\left(\mathbf{1}_{N}^{\prime} \boldsymbol{w}_{t}\right)} .
$$

The last equality of the proposition stems from the simple observation that

$$
\left(\mathbf{1}_{N}^{\prime} \operatorname{diag}\left(\boldsymbol{w}_{t}\right) \mathbf{1}_{N}\right) /\left(\mathbf{1}_{N}^{\prime} \boldsymbol{w}_{t}\right)=1
$$

\section{Appendix B. Proof of Lemma 3}

By definition, at equilibrium, the $\boldsymbol{x}_{t}$ are all equal and so are the returns (given the form of $\boldsymbol{R}$ ). From the first line of (11), we thus have $\boldsymbol{x}^{*}=\boldsymbol{f}\left(\boldsymbol{r}^{*}, \ldots, \boldsymbol{r}^{*}\right)$. Moreover,

$$
\begin{aligned}
\boldsymbol{R}(\boldsymbol{x}, \boldsymbol{x}, \boldsymbol{e}) & =\left(1+\boldsymbol{x}^{\prime}\left(\boldsymbol{e}-\mathbf{1}_{K}\right)\right)\left(\operatorname{diag}(\boldsymbol{x})-\boldsymbol{x} \boldsymbol{x}^{\prime}\right)^{-1} \boldsymbol{x}-\mathbf{1}_{K} \\
& =\left(1+\boldsymbol{x}^{\prime}\left(\boldsymbol{e}-\mathbf{1}_{K}\right)\right)\left(\operatorname{diag}(\boldsymbol{x})^{-1}+\frac{\mathbf{1}_{K} \mathbf{1}_{K}^{\prime}}{1-\boldsymbol{x}^{\prime} \operatorname{diag}(x)^{-1} \boldsymbol{x}}\right) \boldsymbol{x}-\mathbf{1}_{K} \\
& =\left(1+\boldsymbol{x}^{\prime}\left(\boldsymbol{e}-\mathbf{1}_{K}\right)\right)\left(\mathbf{1}_{K}+\frac{\mathbf{1}_{K}\left(\mathbf{1}_{K}^{\prime} \boldsymbol{x}\right)}{1-\mathbf{1}_{K}^{\prime} \boldsymbol{x}}\right)-\mathbf{1}_{K} \\
& =\left(\left(1+\boldsymbol{x}^{\prime}\left(\boldsymbol{e}-\mathbf{1}_{K}\right)\right)\left(1+\frac{\mathbf{1}_{K}^{\prime} \boldsymbol{x}}{1-\mathbf{1}_{K}^{\prime} \boldsymbol{x}}\right)-1\right) \mathbf{1}_{K} \\
& =\left(\left(1+\boldsymbol{x}^{\prime}\left(\boldsymbol{e}-\mathbf{1}_{K}\right)\right)\left(\frac{1}{1-\mathbf{1}_{K}^{\prime} \boldsymbol{x}}\right)-1\right) \mathbf{1}_{K} \\
& =\frac{\boldsymbol{x}^{\prime} \boldsymbol{e}}{1-\boldsymbol{x}^{\prime} \mathbf{1}_{K}} \mathbf{1}_{K},
\end{aligned}
$$

where we have used the Sherman-Morrison identity for matrix inversion (see e.g. Chapter 3 of Meyer (2000)) in the second line. 


\section{Appendix C. Proof of Propositions 4 and 5}

We start with Proposition 4 by considering the case $\boldsymbol{\phi}^{*} \succ \mathbf{0}_{N}$ when all investors survive (i.e., have a strictly positive market share). Quite obviously, at a PCE, the growth rate of rescaled wealths (equal to $\boldsymbol{\rho}^{*}=\left(\boldsymbol{x}^{*}\right)^{\prime}\left(\boldsymbol{r}^{*}+\overline{\boldsymbol{e}}\right)$ ) is constant across agents (if not the shares $\boldsymbol{\phi}$ would not be stationary). This can formally be shown by plugging $\phi_{t+1}=\phi_{t}=\phi^{*}$ in equation (9):

$$
\boldsymbol{\phi}^{*}=\operatorname{diag}\left(\boldsymbol{\phi}^{*}\right) \frac{\mathbf{1}_{N}+\left(\boldsymbol{x}^{*}\right)^{\prime}\left(\boldsymbol{r}^{*}+\overline{\boldsymbol{e}}\right)}{1+\mathbf{1}_{N}^{\prime} \operatorname{diag}\left(\boldsymbol{\phi}^{*}\right)\left(\boldsymbol{x}^{*}\right)^{\prime}\left(\boldsymbol{r}^{*}+\overline{\boldsymbol{e}}\right)},
$$

which implies

$$
\left(\boldsymbol{x}^{*}\right)^{\prime}\left(\boldsymbol{r}^{*}+\overline{\boldsymbol{e}}\right)=\left[\mathbf{1}_{N}^{\prime} \operatorname{diag}\left(\boldsymbol{\phi}^{*}\right)\left(\boldsymbol{x}^{*}\right)^{\prime}\left(\boldsymbol{r}^{*}+\overline{\boldsymbol{e}}\right)\right] \times \mathbf{1}_{N},
$$

which is a vector with constant values. Now, multiplying both sides of the equilibrium version of equation (7) by $\left(\operatorname{diag}\left(\boldsymbol{x}^{*} \boldsymbol{\phi}^{*}\right)-\boldsymbol{x}^{*} \operatorname{diag}\left(\boldsymbol{\phi}^{*}\right)\left(\boldsymbol{x}^{*}\right)^{\prime}\right)$ gives

$$
\begin{aligned}
\left(\operatorname{diag}\left(\boldsymbol{x}^{*} \boldsymbol{\phi}^{*}\right)-\boldsymbol{x}^{*} \operatorname{diag}\left(\boldsymbol{\phi}^{*}\right)\left(\boldsymbol{x}^{*}\right)^{\prime}\right) \boldsymbol{r}^{*}= & \boldsymbol{x}^{*} \operatorname{diag}\left(\boldsymbol{\phi}^{*}\right)\left[\mathbf{1}_{N}+\left(\boldsymbol{x}^{*}\right)^{\prime}\left(\overline{\boldsymbol{e}}-\mathbf{1}_{K}\right)\right] \\
& -\left(\operatorname{diag}\left(\boldsymbol{x}^{*} \boldsymbol{\phi}^{*}\right)-\boldsymbol{x}^{*} \operatorname{diag}\left(\boldsymbol{\phi}^{*}\right)\left(\boldsymbol{x}^{*}\right)^{\prime}\right) \mathbf{1}_{K},
\end{aligned}
$$

which simplifies to

$$
\left(\operatorname{diag}\left(\boldsymbol{x}^{*} \boldsymbol{\phi}^{*}\right)-\boldsymbol{x}^{*} \operatorname{diag}\left(\boldsymbol{\phi}^{*}\right)\left(\boldsymbol{x}^{*}\right)^{\prime}\right) \boldsymbol{r}^{*}=\boldsymbol{x}^{*} \operatorname{diag}\left(\boldsymbol{\phi}^{*}\right)\left(\boldsymbol{x}^{*}\right)^{\prime} \overline{\boldsymbol{e}}
$$

because of identity (A.4). This further leads to

$$
\boldsymbol{r}^{*}=\left(\operatorname{diag}\left(\boldsymbol{x}^{*} \boldsymbol{\phi}^{*}\right)-\boldsymbol{x}^{*} \operatorname{diag}\left(\boldsymbol{\phi}^{*}\right)\left(\boldsymbol{x}^{*}\right)^{\prime}\right)^{-1} \boldsymbol{x}^{*} \operatorname{diag}\left(\boldsymbol{\phi}^{*}\right)\left(\boldsymbol{x}^{*}\right)^{\prime} \overline{\boldsymbol{e}},
$$

which is obvious given the second line of (7). This equality can be viewed as the multivariate equivalent of equation (51) in Anufriev and Bottazzi (2010). Now, equation (C.3) is equivalent to

$$
\boldsymbol{x}^{*} \operatorname{diag}\left(\boldsymbol{\phi}^{*}\right)\left(\boldsymbol{x}^{*}\right)^{\prime}\left(\boldsymbol{r}^{*}+\overline{\boldsymbol{e}}\right)=\operatorname{diag}\left(\boldsymbol{x}^{*} \boldsymbol{\phi}^{*}\right) \boldsymbol{r}^{*},
$$

and hence plugging equation (C.2) into this expression gives

$$
\left[\mathbf{1}_{N}^{\prime} \operatorname{diag}\left(\boldsymbol{\phi}^{*}\right)\left(\boldsymbol{x}^{*}\right)^{\prime}\left(\boldsymbol{r}^{*}+\overline{\boldsymbol{e}}\right)\right] \times \boldsymbol{x}^{*} \operatorname{diag}\left(\boldsymbol{\phi}^{*}\right) \mathbf{1}_{N}=\operatorname{diag}\left(\boldsymbol{x}^{*} \boldsymbol{\phi}^{*}\right) \boldsymbol{r}^{*} .
$$

Since the inverse of a diagonal matrix with nonzero terms ${ }^{13}$ is well defined, we get

$$
\boldsymbol{r}^{*}=\left[\mathbf{1}_{N}^{\prime} \operatorname{diag}\left(\boldsymbol{\phi}^{*}\right)\left(\boldsymbol{x}^{*}\right)^{\prime}\left(\boldsymbol{r}^{*}+\overline{\boldsymbol{e}}\right)\right] \times \operatorname{diag}\left(\boldsymbol{x}^{*} \boldsymbol{\phi}^{*}\right)^{-1} \boldsymbol{x}^{*} \operatorname{diag}\left(\boldsymbol{\phi}^{*}\right) \mathbf{1}_{N},
$$

which, given identity (A.4), simplifies to

$$
\boldsymbol{r}^{*}=\left[\mathbf{1}_{N}^{\prime} \operatorname{diag}\left(\phi^{*}\right)\left(\boldsymbol{x}^{*}\right)^{\prime}\left(\boldsymbol{r}^{*}+\overline{\boldsymbol{e}}\right)\right] \times \mathbf{1}_{K},
$$

so that $\boldsymbol{r}^{*}$ has the form $\boldsymbol{r}^{*}=\alpha \mathbf{1}_{K}$. Multiplying both sides by $\mathbf{1}_{K}^{\prime}$, we get

$$
\alpha K=K\left[\mathbf{1}_{N}^{\prime} \operatorname{diag}\left(\boldsymbol{\phi}^{*}\right)\left(\boldsymbol{x}^{*}\right)^{\prime}\left(\alpha \mathbf{1}_{K}+\overline{\boldsymbol{e}}\right)\right],
$$

that is:

$$
\alpha=\frac{\mathbf{1}_{N}^{\prime} \operatorname{diag}\left(\boldsymbol{\phi}^{*}\right)\left(\boldsymbol{x}^{*}\right)^{\prime} \overline{\boldsymbol{e}}}{1-\mathbf{1}_{N}^{\prime} \operatorname{diag}\left(\boldsymbol{\phi}^{*}\right)\left(\boldsymbol{x}^{*}\right)^{\prime} \mathbf{1}_{K}} .
$$

\footnotetext{
${ }^{13}$ We are assuming nonzero aggregate demands for all assets.
} 
When only $N^{\diamond}<N$ investors survive, then the same analysis can be carried out on the truncated vector $\boldsymbol{\phi}^{\diamond} \succ \mathbf{0}_{N^{\diamond}}$ and truncated demands matrix $\boldsymbol{x}^{\diamond}$ in which the demands of the vanishing agents were removed.

The proof of Proposition 5 follows the same lines. The only problem is that zeros appears in the r.h.s. of Equation (C.5) unless we restrict it to $\boldsymbol{x}^{ \pm}$and $\boldsymbol{r}^{ \pm}$, which gives the first statement of the proposition (the two versions of $\boldsymbol{r}^{ \pm}$in equation (19) are equal because the additional lines in the second expression correspond to the assets with zero demand and thus have no impact). The second statement stems from the equality (C.4). Its last $K^{0}$ lines (with zeros in the r.h.s) can be written, given the decomposition (18),

$$
\boldsymbol{\Lambda}^{0, \pm}\left(\boldsymbol{r}^{ \pm}+\overline{\boldsymbol{e}}^{ \pm}\right)+\boldsymbol{\Lambda}^{0}\left(\boldsymbol{r}^{0}+\overline{\boldsymbol{e}}^{0}\right)=\mathbf{0}_{K^{0}}
$$

from which the second claim of the proposition follows. We recall that $\Lambda^{0, \pm}$ is a $\left(K^{0} \times K^{ \pm}\right)$matrix so that the dimensions indeed match.

\section{Appendix D. Proof of Propositions 6 and 7}

Proof of Proposition 6. The first two expressions are deduced from the structure of $\boldsymbol{\Phi}$ and $\boldsymbol{R}$ in Equation (13). Because the function $\boldsymbol{R}$ does not depend on the elements of the vector $\boldsymbol{r}$, we have

$$
\frac{\partial r_{t+1}^{(k)}}{\partial r_{t}^{(i)}}=0 .
$$

And the upper left block of $\boldsymbol{J}$ is a $K \times K$ matrix filled with zeros. We also have

$$
\frac{\partial \phi_{t+1, n}}{\partial r_{t}^{(k)}}=\sum_{i=1}^{K} \frac{\partial \phi_{t+1, n}}{\partial r_{t+1}^{(i)}} \frac{\partial r_{t+1}^{(i)}}{\partial r_{t}^{(k)}}=0,
$$

because, inside the sum, the partial derivatives involved at the right side of the product are zero. Consequently, the lower left block of $\boldsymbol{J}$ is a $N \times K$ matrix filled with zeros.

We now consider elements of the upper right block. We begin with an expression for the columns of this matrix. The column $n$ is obtained as $\frac{\partial \boldsymbol{r}_{t+1}}{\partial \phi_{t, n}}$, with

$$
\boldsymbol{r}_{t+1}=\boldsymbol{A}\left(\boldsymbol{x}, \phi_{t}\right)^{-1} \boldsymbol{B}\left(\boldsymbol{x}, \phi_{t}\right) \overline{\boldsymbol{e}},
$$

where, $\boldsymbol{A}, \boldsymbol{B}$ and $\boldsymbol{C}$ are $K \times K$ matrices respectively written

$$
\begin{aligned}
& \boldsymbol{A}\left(x, \phi_{t}\right)=\left(\boldsymbol{C}\left(\boldsymbol{x}, \boldsymbol{\phi}_{t}\right)-\boldsymbol{B}\left(\boldsymbol{x}, \boldsymbol{\phi}_{t}\right)\right), \\
& \boldsymbol{B}\left(x, \phi_{t}\right)=\boldsymbol{x} \operatorname{diag}\left(\boldsymbol{\phi}_{t}\right) \boldsymbol{x}^{\prime}, \quad \boldsymbol{C}\left(\boldsymbol{x}, \boldsymbol{\phi}_{t}\right)=\operatorname{diag}\left(\boldsymbol{x} \phi_{t}\right) .
\end{aligned}
$$

The column $n$ is obtained as

$$
\begin{aligned}
\frac{\partial \boldsymbol{r}_{t+1}}{\partial \phi_{t, n}} & =\frac{\partial\left(\boldsymbol{A}^{-1}\right)}{\partial \phi_{t, n}} \boldsymbol{B} \overline{\boldsymbol{e}}+\boldsymbol{A}^{-1} \frac{\partial \boldsymbol{B}}{\partial \phi_{t, n}} \overline{\boldsymbol{e}} \\
& =-\boldsymbol{A}^{-1} \frac{\partial \boldsymbol{A}}{\partial \phi_{t, n}} \boldsymbol{A}^{-1} \boldsymbol{B} \overline{\boldsymbol{e}}+\boldsymbol{A}^{-1} \frac{\partial \boldsymbol{B}}{\partial \phi_{t, n}} \overline{\boldsymbol{e}} \\
& =-\boldsymbol{A}^{-1}\left(\frac{\partial \boldsymbol{C}}{\partial \phi_{t, n}}-\frac{\partial \boldsymbol{B}}{\partial \phi_{t, n}}\right) \boldsymbol{r}_{t+1}+\boldsymbol{A}^{-1} \frac{\partial \boldsymbol{B}}{\partial \phi_{t}^{n}} \overline{\boldsymbol{e}} \\
& =\boldsymbol{D}\left(\frac{\partial \boldsymbol{B}}{\partial \phi_{t, n}}\left(\boldsymbol{r}_{t+1}+\overline{\boldsymbol{e}}\right)-\frac{\partial \boldsymbol{C}}{\partial \phi_{t, n}} \boldsymbol{r}_{t+1}\right)
\end{aligned}
$$


with $\boldsymbol{D}=\boldsymbol{A}^{-1}$. We have used the formula for the derivative of the inverse of a matrix that can be found in Magnus and Neudecker (2007) (see Chapter 8, Section 4 Theorem 3 p. 171).

Element-wise, the vector expression obtained above becomes

$$
\frac{\partial r_{t+1}^{(k)}}{\partial \phi_{t, n}}=\sum_{i=1}^{K} d_{k i}\left(\sum_{l=1}^{K} x_{n}^{(i)} x_{n}^{(l)}\left(r_{t+1}^{(l)}+e^{(l)}\right)-x_{n}^{(l)} r_{t+1}^{(l)}\right),
$$

where the $d_{k i}$ are the elements of $\boldsymbol{D}$. This partial derivative depends on $\phi_{t, n}$ via the $k^{\text {th }}$ line of $D$. This gives the upper right block of $\boldsymbol{J}$.

Given the structure of $\boldsymbol{\Phi}$, the $n^{\text {th }}$ component of $\phi_{t+1}$ depends on $\phi_{t}$ via $\phi_{t}$ itself but also via $\boldsymbol{r}_{t+1}$. This component is written

$$
\phi_{t+1, n}=\frac{\phi_{t, n}\left(1+\sum_{k=1}^{K} x_{n}^{(k)}\left(r_{t+1}^{(k)}+\bar{e}^{(k)}\right)\right)}{1+\sum_{q=1}^{N} \sum_{k=1}^{K} \phi_{t, q} x_{q}^{(k)}\left(r_{t+1}^{(k)}+\bar{e}^{(k)}\right)} .
$$

The elements of the lower right block are equal to

$$
\frac{\partial \phi_{t+1, n}}{\partial \phi_{t, q}}+\sum_{k=1}^{K} \frac{\partial \phi_{t+1, n}}{\partial r_{t+1}^{(k)}} \frac{\partial r_{t+1}^{(k)}}{\partial \phi_{t, q}}, \quad \text { for } n, q=1, \ldots, N
$$

Among the three partial derivatives involved in the above expression, we still need to compute two. For the partial derivative in the middle, we have

$$
\begin{aligned}
\frac{\partial \phi_{t+1, n}}{\partial r_{t+1}^{(k)}} & =\frac{\phi_{t, n} x_{n}^{(k)}}{1+\sum_{q=1}^{N} \sum_{l=1}^{K} \phi_{t, q} x_{q}^{(l)}\left(r_{t+1}^{(l)}+\bar{e}^{(l)}\right)}-\frac{\left(\sum_{q=1}^{N} \phi_{t, q} x_{q}^{(k)}\right)\left(\phi_{t, n}\left(1+\sum_{l=1}^{K} x_{n}^{(l)}\left(r_{t+1}^{(l)}+\bar{e}^{(l)}\right)\right)\right)}{\left(1+\sum_{q=1}^{N} \sum_{l=1}^{K} \phi_{t, q} x_{q}^{(l)}\left(r_{t+1}^{(l)}+\bar{e}^{(l)}\right)\right)^{2}} \\
& =\phi_{t, n}\left(\frac{x_{n}^{(k)}}{1+\sum_{q=1}^{N} \sum_{l=1}^{K} \phi_{t, q} x_{q}^{(l)}\left(r_{t+1}^{(l)}+\bar{e}^{(l)}\right)}-\frac{\left(\sum_{q=1}^{N} \phi_{t, q} x_{q}^{(k)}\right)\left(1+\sum_{l=1}^{K} x_{n}^{(l)}\left(r_{t+1}^{(l)}+\bar{e}^{(l)}\right)\right)}{\left(1+\sum_{q=1}^{N} \sum_{l=1}^{K} \phi_{t, q} x_{q}^{(l)}\left(r_{t+1}^{(l)}+\bar{e}^{(l)}\right)\right)^{2}}\right) \\
& =\phi_{t, n}\left(\frac{x_{n}^{(k)}\left(1+\sum_{q=1}^{N} \sum_{l=1}^{K} \phi_{t, q} x_{q}^{(l)}\left(r_{t+1}^{(l)}+\bar{e}^{(l)}\right)\right)-\left(\sum_{q=1}^{N} \phi_{t}^{q} x_{q}^{(k)}\right)\left(1+\sum_{l=1}^{K} x_{n}^{(l)}\left(r_{t+1}^{(l)}+\bar{e}^{(l)}\right)\right)}{\left(1+\sum_{q=1}^{N} \sum_{l=1}^{K} \phi_{t, q} x_{q}^{(l)}\left(r_{t+1}^{(l)}+\bar{e}^{(l)}\right)\right)^{2}}\right)
\end{aligned}
$$

The last partial derivative to be computed (left side of the initial expression) is obtained as

$$
\begin{aligned}
\frac{\partial \phi_{t+1, n}}{\partial \phi_{t, q}}= & \frac{\left(1+\sum_{k=1}^{K} x_{n}^{(k)}\left(r_{t+1}^{(k)}+\bar{e}^{(k)}\right)\right)}{1+\sum_{v=1}^{N} \sum_{k=1}^{K} \phi_{t, v} x_{v}^{(k)}\left(r_{t+1}^{(k)}+\bar{e}^{(k)}\right)} \delta_{n q} \\
& -\frac{\phi_{t, n}\left(\sum_{k=1}^{K} x_{q}^{(k)}\left(r_{t+1}^{(k)}+\bar{e}^{(k)}\right)\right)\left(1+\sum_{k=1}^{K} x_{n}^{(k)}\left(r_{t+1}^{(k)}+\bar{e}^{(k)}\right)\right)}{\left(1+\sum_{v=1}^{N} \sum_{k=1}^{K} \phi_{t, v} x_{v}^{(k)}\left(r_{t+1}^{(k)}+\bar{e}^{(k)}\right)\right)^{2}} \\
= & \frac{1+\sum_{k=1}^{K} x_{n}^{(k)}\left(r_{t+1}^{(k)}+\bar{e}^{(k)}\right)}{1+\sum_{v=1}^{N} \sum_{k=1}^{K} \phi_{t, v} x_{v}^{(k)}\left(r_{t+1}^{(k)}+\bar{e}^{(k)}\right)}\left(\delta_{n q}-\phi_{t, n} \frac{\sum_{k=1}^{K} x_{q}^{(k)}\left(r_{t+1}^{(k)}+\bar{e}^{(k)}\right)}{1+\sum_{v=1}^{N} \sum_{k=1}^{K} \phi_{t, v} x_{v}^{(k)}\left(r_{t+1}^{(k)}+\bar{e}^{(k)}\right)}\right)
\end{aligned}
$$

where $\delta_{n q}$ is the Kronecker delta ( 1 if $n=q, 0$ otherwise). Gathering the terms completes the proof. 
Proof of Proposition 7. The proof starts with the expression in Proposition 6 and proceeds with simplifications stemming from relationships holding at equilibrium. They are based on the expressions (15) and (16), obtained in Section 3.1 where the PCE is studied. At equilibrium, we have for $n=1, \ldots, N$

$$
\sum_{k=1}^{K} x_{n}^{(k)}\left(r_{t+1}^{(k)}+\bar{e}^{(k)}\right)=r^{*}
$$

where $r^{*}$ is the common value of price return for all assets. We also have, from $\sum_{n=1}^{N} \phi_{t, n}=1$, so that

$$
1+\sum_{n=1}^{N} \sum_{k=1}^{K} \phi_{t, n} x_{n}^{(k)}\left(r_{t+1}^{(k)}+\bar{e}^{(k)}\right)=1+r^{*} .
$$

The elements of the two matrices on the left side of $\boldsymbol{J}$ are all zeros. The elements of $\mathcal{T}\left(\boldsymbol{S}^{*}\right)$ are explicitly given by

$$
\begin{aligned}
\frac{\partial r_{t+1}^{(k)}}{\partial \phi_{t, n}}\left(\boldsymbol{S}^{*}\right) & =\sum_{i=1}^{K} d_{k i}\left(\sum_{l=1}^{K} x_{n}^{(i)} x_{n}^{(l)}\left(r_{t+1}^{(l)}+\bar{e}^{(l)}\right)-x_{n}^{(l)} r_{t+1}^{(l)}\right) \\
& =\sum_{i=1}^{K} d_{k i}\left(x_{n}^{(i)} \sum_{l=1}^{K} x_{n}^{(l)}\left(r_{t+1}^{(l)}+\bar{e}^{(l)}\right)-\sum_{l=1}^{K} x_{n}^{(l)} r_{t+1}^{(l)}\right) \\
& =\sum_{i=1}^{K} d_{k i}\left(r^{*} x_{n}^{(i)}-r^{*} \sum_{l=1}^{K} x_{n}^{(l)}\right) \\
& =-r^{*} \sum_{i=1}^{K} d_{k i} \sum_{\substack{l=1 \\
l \neq i}}^{K} x_{n}^{(l)},
\end{aligned}
$$

where the $d_{k i}$ are the elements of the matrix $\boldsymbol{D}^{*}=\left(\operatorname{diag}\left(\boldsymbol{x} \boldsymbol{\phi}^{*}\right)-\boldsymbol{x} \operatorname{diag}\left(\boldsymbol{\phi}^{*}\right) \boldsymbol{x}^{\prime}\right)^{-1}$. We now turn to the elements of $\mathcal{F}\left(\boldsymbol{S}^{*}\right)$. They are obtained as follows

$$
\begin{aligned}
& \frac{\partial \phi_{t+1, n}}{\partial \phi_{t, q}}\left(\boldsymbol{S}^{*}\right)=\frac{1+\sum_{k=1}^{K} x_{n}^{(k)}\left(r_{t+1}^{(k)}+\bar{e}^{(k)}\right)}{1+\sum_{v=1}^{N} \sum_{k=1}^{K} \phi_{t, v} x_{v}^{(k)}\left(r_{t+1}^{(k)}+\bar{e}^{(k)}\right)}\left(\delta_{n q}-\phi_{t, n} \frac{\sum_{k=1}^{K} x_{q}^{(k)}\left(r_{t+1}^{(k)}+\bar{e}^{(k)}\right)}{1+\sum_{v=1}^{N} \sum_{k=1}^{K} \phi_{t, v} x_{v}^{(k)}\left(r_{t+1}^{(k)}+\bar{e}^{(k)}\right)}\right) \\
& +\phi_{t, n} \sum_{k=1}^{K}\left[\frac{x_{n}^{(k)}\left(1+\sum_{v=1}^{N} \sum_{l=1}^{K} \phi_{t, v} x_{v}^{(l)}\left(r_{t+1}^{(l)}+\bar{e}^{(l)}\right)\right)-\left(\sum_{v=1}^{N} \phi_{t, v} x_{v}^{(k)}\right)\left(1+\sum_{l=1}^{K} x_{n}^{(l)}\left(r_{t+1}^{(l)}+\bar{e}^{(l)}\right)\right)}{\left(1+\sum_{v=1}^{N} \sum_{l=1}^{K} \phi_{t, v} x_{v}^{(l)}\left(r_{t+1}^{(l)}+\bar{e}^{(l)}\right)\right)^{2}}\right. \\
& \left.\times \sum_{i=1}^{K} d_{k i}\left(\sum_{l=1}^{K} x_{q}^{(i)} x_{q}^{(l)}\left(r_{t+1}^{(l)}+\bar{e}^{(l)}\right)-x_{q}^{(l)} r_{t+1}^{(l)}\right)\right] \\
& =\frac{1+r^{*}}{1+r^{*}}\left(\delta_{n q}-\phi_{t, n} \frac{r^{*}}{1+r^{*}}\right)+\phi_{t, n} \sum_{k=1}^{K}\left[\frac{x_{n}^{(k)}\left(1+r^{*}\right)-\left(\sum_{v=1}^{N} \phi_{t, v} x_{v}^{(k)}\right)\left(1+r^{*}\right)}{\left(1+r^{*}\right)^{2}}\left(-r^{*}\right) \sum_{i=1}^{K} d_{k i} \sum_{\substack{l=1 \\
l \neq i}}^{K} x_{q}^{(l)}\right] \\
& =\delta_{n q}-\frac{r^{*} \phi_{n}^{*}}{1+r^{*}}\left[1+\sum_{k=1}^{K}\left(x_{n}^{(k)}-\sum_{v=1}^{N} \phi_{v}^{*} x_{v}^{(k)}\right) \sum_{i=1}^{K} d_{k i} \sum_{\substack{l=1 \\
l \neq i}}^{K} x_{q}^{(l)}\right]
\end{aligned}
$$

where in the last line we have replaced $\phi_{t}$ by the equilibrium notation $\phi^{*}$. 


\section{Appendix E. Proof of Lemma 8}

From the second part of (7), we get

$$
\boldsymbol{r}_{t+1}=\left(\operatorname{diag}\left(\boldsymbol{x} \phi_{t}\right)-\boldsymbol{x} \operatorname{diag}\left(\phi_{t}\right) \boldsymbol{x}^{\prime}\right)^{-1}\left(\boldsymbol{x} \operatorname{diag}\left(\boldsymbol{\phi}_{t}\right) \boldsymbol{x}^{\prime} \boldsymbol{e}_{t+1}\right),
$$

so that

$$
\left(\operatorname{diag}\left(\boldsymbol{x} \phi_{t}\right)-\boldsymbol{x} \operatorname{diag}\left(\phi_{t}\right) \boldsymbol{x}^{\prime}\right) \boldsymbol{r}_{t+1}=\boldsymbol{x} \operatorname{diag}\left(\phi_{t}\right) \boldsymbol{x}^{\prime} \boldsymbol{e}_{t+1},
$$

hence adding and subtracting $\operatorname{diag}\left(\boldsymbol{x} \boldsymbol{\phi}_{t}\right) \boldsymbol{e}_{t+1}$ on the r.h.s. implies

$$
\boldsymbol{r}_{t+1}+\boldsymbol{e}_{t+1}=\left(\operatorname{diag}\left(\boldsymbol{x} \phi_{t}\right)-\boldsymbol{x} \operatorname{diag}\left(\boldsymbol{\phi}_{t}\right) \boldsymbol{x}^{\prime}\right)^{-1} \operatorname{diag}\left(\boldsymbol{x} \phi_{t}\right) \boldsymbol{e}_{t+1} .
$$

Plugging this into (9) yields the first result. Then, multiplying both sides of (9) by $\boldsymbol{x}$, we get

$$
\boldsymbol{z}_{t+1}=\boldsymbol{x} \operatorname{diag}\left(\phi_{t}\right) \times \frac{\mathbf{1}_{N}+\boldsymbol{x}^{\prime}\left(\boldsymbol{r}_{t+1}+\boldsymbol{e}_{t+1}\right)}{1+\mathbf{1}_{N}^{\prime} \operatorname{diag}\left(\phi_{t}\right) \boldsymbol{x}^{\prime}\left(\boldsymbol{r}_{t+1}+\boldsymbol{e}_{t+1}\right)}=\frac{\boldsymbol{z}_{t}+\boldsymbol{x} \operatorname{diag}\left(\boldsymbol{\phi}_{t}\right) \boldsymbol{x}^{\prime}\left(\boldsymbol{r}_{t+1}+\boldsymbol{e}_{t+1}\right)}{1+\boldsymbol{z}_{t}^{\prime}\left(\boldsymbol{r}_{t+1}+\boldsymbol{e}_{t+1}\right)} .
$$

From (E.1), we have that

$$
\left(\boldsymbol{x} \operatorname{diag}\left(\phi_{t}\right) \boldsymbol{x}^{\prime}\right)\left(\boldsymbol{r}_{t+1}+\boldsymbol{e}_{t+1}\right)=\operatorname{diag}\left(\boldsymbol{x} \phi_{t}\right) \boldsymbol{r}_{t+1}=\operatorname{diag}\left(\boldsymbol{z}_{t}\right) \boldsymbol{r}_{t+1},
$$

so that combining both expressions above,

$$
\boldsymbol{z}_{t+1}=\operatorname{diag}\left(\boldsymbol{z}_{t}\right) \frac{\boldsymbol{r}_{t+1}+\mathbf{1}_{K}}{1+\boldsymbol{z}_{t}^{\prime}\left(\boldsymbol{r}_{t+1}+\boldsymbol{e}_{t+1}\right)}
$$

The final statement of the lemma is obvious given this expression.

\section{Appendix F. Properties of M}

The matrix $\boldsymbol{M}$ defined in (36) will play a crucial role in subsequent proofs, in this section, we unveil some of its properties. In the remainder of the proofs $[\boldsymbol{M}]_{i j}$ and $[\boldsymbol{v}]_{i}$ are the straightforward notations for the elements of matrices and vectors. We omit the time-dependence of $\boldsymbol{M}$ for notational convenience. The elements of $\boldsymbol{M}$ are equal to

$$
\left[\operatorname{diag}\left(\boldsymbol{x} \phi_{t}\right)^{-1} \boldsymbol{x} \operatorname{diag}\left(\phi_{t}\right) \boldsymbol{x}^{\prime}\right]_{i j}=\frac{\sum_{l=1}^{N} \phi_{t, l} x_{l}^{(i)} x_{l}^{(j)}}{\sum_{n=1}^{N} \phi_{t, n} x_{n}^{(i)}} .
$$

One first property of this matrix is that when $\phi_{t, l}$ converges to 1 (and all other $\phi_{t, i}$ to zero), $\boldsymbol{M}$ converges to the $(K \times K)$ matrix

$$
\boldsymbol{M}(l)=\left(\begin{array}{c}
\boldsymbol{x}_{l}^{\prime} \\
\vdots \\
\boldsymbol{x}_{l}^{\prime}
\end{array}\right)=\left(\begin{array}{ccc}
x_{l}^{(1)} & \ldots & x_{l}^{(K)} \\
\vdots & & \vdots \\
x_{l}^{(1)} & \ldots & x_{l}^{(K)}
\end{array}\right),
$$

where the rows are all identical and $\boldsymbol{x}_{l}$ denotes the $l$-th column of the matrix $\boldsymbol{x}$. We recall that $\lambda_{i}=\sum_{k=1}^{K} x_{i}^{(k)}<1$ is the leverage of the $i^{t h}$ agent. The following lemma provides additional properties of $\boldsymbol{M}$.

Lemma 12. Under (6), the following holds:

i) the row sums of $\boldsymbol{M}$ (and thus all of its elements) are strictly smaller than some constant $\nu<1$. Thus, $\lim _{q \rightarrow \infty} \boldsymbol{M}^{q}=\mathbf{0}_{K, K}$. 
ii) $\exists \kappa>0$ such that $\forall \delta(\epsilon)>0, \quad \phi_{t, l} \geq 1-\delta(\epsilon) \quad \Longrightarrow \quad \sum_{j=1}^{K}\left|M_{i j}-M(l)_{i j}\right| \leq \kappa \delta(\epsilon), \forall i$,

iii) for any small $\epsilon>0$, there exists $\delta(\epsilon)>0$ such that

$$
\phi_{t, l} \geq 1-\delta(\epsilon) \Longrightarrow\left|\left[\sum_{q=1}^{\infty} \boldsymbol{M}^{q}-\boldsymbol{M}(l)^{q}\right]_{i j}\right| \leq \epsilon, \quad \forall(i, j)
$$

All inequalities are meant element-wise.

Proof. Proof of $i$ ). First of all, all elements of $\boldsymbol{M}$ are positive if we assume that (6) holds. The column sums are equal to

$$
\sum_{j=1}^{K} \frac{\sum_{l=1}^{N} \phi_{t, l} x_{l}^{(i)} x_{l}^{(j)}}{\sum_{n=1}^{N} \phi_{t, n} x_{n}^{(i)}}=\frac{\sum_{l=1}^{N} \phi_{t, l} x_{l}^{(i)} \lambda_{l}}{\sum_{n=1}^{N} \phi_{t, n} x_{n}^{(i)}}
$$

where by (6) $i i$ ) the leverages $\lambda_{l}$ are strictly smaller than one. Hence, the ratio is indeed smaller than one and we denote $\nu<1$ the largest of these ratios. Since all elements of $\boldsymbol{M}$ are positive, all elements must also be smaller than $\nu$. Moreover, the elements of $\boldsymbol{M}^{2}$ satisfy

$$
\left[\boldsymbol{M}^{2}\right]_{i j}=\sum_{k=1}^{K} M_{i k} M_{k j} \leq \max _{k} M_{k j} \sum_{k=1}^{K} M_{i k} \leq \nu^{2},
$$

and iteratively we get $\left[\boldsymbol{M}^{q}\right]_{i j} \leq \nu^{q}$, hence the limit $\lim _{q \rightarrow \infty} \boldsymbol{M}^{q}=\mathbf{0}_{K, K}$ holds.

Proof of $i i)$. We have

$$
\begin{aligned}
M_{i j}-x_{l}^{(j)} & =\frac{\sum_{n=1}^{N} \phi_{t, n} x_{n}^{(i)} x_{n}^{(j)}}{\sum_{n=1}^{N} \phi_{t, n} x_{n}^{(i)}}-x_{l}^{(j)} \\
& =\frac{\sum_{n \neq l} \phi_{t, n} x_{n}^{(i)}\left(x_{n}^{(j)}-x_{l}^{(j)}\right)}{\sum_{n=1}^{N} \phi_{t, n} x_{n}^{(i)}}
\end{aligned}
$$

therefore

$$
\sum_{j=1}^{K}\left|M_{i j}-x_{l}^{(j)}\right| \leq \sum_{j=1}^{K} \frac{\sum_{n \neq l} \phi_{t, n} x_{n}^{(i)}\left|x_{n}^{(j)}-x_{l}^{(j)}\right|}{\sum_{n=1}^{N} \phi_{t, n} x_{n}^{(i)}}=\frac{\sum_{n \neq l} \phi_{t, n} x_{n}^{(i)} \sum_{j=1}^{K}\left|x_{n}^{(j)}-x_{l}^{(j)}\right|}{\sum_{n=1}^{N} \phi_{t, n} x_{n}^{(i)}}
$$

so that when $\phi_{t, l} \geq 1-\delta(\epsilon) \Leftrightarrow \sum_{n \neq l} \phi_{t, n} \leq \delta(\epsilon)$, we have, since sums of demands $\sum_{k=1}^{K} x_{n}^{(k)}$ are strictly smaller than one,

$$
\sum_{j=1}^{K}\left|M_{i j}-M(l)_{i j}\right| \leq \frac{2 \delta(\epsilon)}{\sum_{n=1}^{N} \phi_{t, n} x_{n}^{(i)}} \leq 2 \kappa \delta(\epsilon),
$$

where $\kappa$ is the largest possible value of the inverse aggregate demands $\left(\sum_{n=1}^{N} \phi_{t, n} x_{n}^{(i)}\right)^{-1}$, which, by (6) is bounded. This completes the proof of the first implication. We underline that the above inequality implies the weaker inequality

$$
\left|M_{i j}-M(l)_{i j}\right| \leq 2 \kappa \delta(\epsilon) .
$$


Proof of iii). We have

$$
\begin{aligned}
\left|\left[\boldsymbol{M}^{2}-\boldsymbol{M}(l)^{2}\right]_{i j}\right| & =\left|\sum_{k=1}^{K}\left\{M_{i k} M_{k j}-M(l)_{i k} M(l)_{k j}\right\}\right| \\
& =\left|\sum_{k=1}^{K}\left\{M_{i k} M_{k j}-x_{l}^{(k)} x_{l}^{(j)}\right\}\right| \\
& =\left|\sum_{k=1}^{K}\left\{M_{k j}\left(M_{i k}-x_{l}^{(k)}\right)+x_{l}^{(k)}\left(M_{k j}-x_{l}^{(j)}\right)\right\}\right| \\
& \leq \sum_{k=1}^{K}\left|M_{i k}-x_{l}^{(k)}\right|+\sum_{k=1}^{K} x_{l}^{(k)}\left|M_{k j}-x_{l}^{(j)}\right| .
\end{aligned}
$$

In the last line, we have used the inequality $M_{k j}<\nu<1$. The inequalities (F.4), (F.5) and $\sum_{k=1}^{K} x_{l}^{(k)}<1$ lead to $\left|\left[\boldsymbol{M}^{2}-\boldsymbol{M}(l)^{2}\right]_{i j}\right| \leq 4 \kappa \delta(\epsilon)$ and recursively, it holds that

$$
\left|\left[\boldsymbol{M}^{q}-\boldsymbol{M}(l)^{q}\right]_{i j}\right| \leq 2^{q} \kappa \delta(\epsilon) .
$$

We now use the fact that both $\boldsymbol{M}^{q}$ and $\boldsymbol{M}(l)$ converge to $\mathbf{0}_{K, K}$ when $q \rightarrow \infty$ (the proof for $\boldsymbol{M}(l)$ is the same as for $\boldsymbol{M})$. For a given $\epsilon$, we consider the largest $n$, which we call $n(\epsilon)$, such that for all $i$ and $j$,

$$
\left|\sum_{q=n(\epsilon)+1}^{\infty}\left[\boldsymbol{M}^{q}-\boldsymbol{M}(l)^{q}\right]_{i j}\right| \leq \epsilon / 2 .
$$

The other half of the series satisfies

$$
\left|\sum_{q=1}^{n(\epsilon)}\left[\boldsymbol{M}^{q}-\boldsymbol{M}(l)^{q}\right]_{i j}\right| \leq \kappa \delta(\epsilon) \sum_{q=1}^{n(\epsilon)} 2^{q} \leq \kappa \delta(\epsilon) 2^{n(\epsilon)+1}
$$

To reach the desired threshold, it suffices to set $\delta(\epsilon)<\frac{\epsilon}{\kappa 2^{n(\epsilon)+2}}$.

\section{Appendix G. Proof of Proposition 9}

We start with the final statement. If (6) is satisfied (nonzero aggregate demands), $\boldsymbol{\rho}_{t+1}$ has only strictly positive elements which means that vanishing agents disappear slowly and that their market shares reach zero only asymptotically. Upon equilibrium, we are in the case of uniform returns $\boldsymbol{r}^{*}=r^{*} \mathbf{1}_{K}$ and plugging this in (C.4), we get

$$
\boldsymbol{x}^{*}\left(\operatorname{diag}\left(\boldsymbol{\phi}^{*}\right)\left(\boldsymbol{x}^{*}\right)^{\prime}\left(r^{*} \mathbf{1}_{K}+\overline{\boldsymbol{e}}\right)-r^{*} \boldsymbol{\phi}^{*}\right)=\mathbf{0}_{\boldsymbol{K}} .
$$

If $K \geq N$, given the number of equations and the number of variables in the above system, it must hold that

$$
\operatorname{diag}\left(\boldsymbol{\phi}^{*}\right)\left(\boldsymbol{x}^{*}\right)^{\prime}\left(r^{*} \mathbf{1}_{K}+\overline{\boldsymbol{e}}\right)-r^{*} \boldsymbol{\phi}^{*}=\mathbf{0}_{\boldsymbol{N}} .
$$

Recalling that $\boldsymbol{\phi}^{*}=\operatorname{diag}\left(\boldsymbol{\phi}^{*}\right) \mathbf{1}_{N}$, this implies

$$
\operatorname{diag}\left(\boldsymbol{\phi}^{*}\right)\left[\left(\boldsymbol{x}^{*}\right)^{\prime}\left(r^{*} \mathbf{1}_{K}+\overline{\boldsymbol{e}}\right)-r^{*} \mathbf{1}_{N}\right]=\mathbf{0}_{\boldsymbol{N}} .
$$

Now let us assume that all $N$ agents survive which yields $\left(\boldsymbol{x}^{*}\right)^{\prime}\left(r^{*} \mathbf{1}_{K}+\overline{\boldsymbol{e}}\right)-r^{*} \mathbf{1}_{N}=\mathbf{0}_{\boldsymbol{N}}$. This is equivalent to

$$
\boldsymbol{R}=\operatorname{diag}\left(\mathbf{1}_{N}-\boldsymbol{x}^{\prime} \mathbf{1}_{K}\right)^{-1} \boldsymbol{x}^{\prime} \overline{\boldsymbol{e}}=r^{*} \mathbf{1}_{N}
$$


i.e., all characteristic ratios are equal. If less than $N$ agents survive, the reasoning is the same, on the sub-matrices pertaining to these agents.

The expression in the proposition stems directly from (32) and (E.2) which we re-write as

$$
\begin{aligned}
\boldsymbol{\phi}_{t+1} & =\operatorname{diag}\left(\boldsymbol{\phi}_{t}\right) \times \frac{\mathbf{1}_{N}+\boldsymbol{x}^{\prime}\left(\boldsymbol{I}_{K}-\operatorname{diag}\left(\boldsymbol{x} \boldsymbol{\phi}_{t}\right)^{-1} \boldsymbol{x} \operatorname{diag}\left(\boldsymbol{\phi}_{t}\right) \boldsymbol{x}^{\prime}\right)^{-1} \boldsymbol{e}_{t+1}}{1+\boldsymbol{\phi}_{t}^{\prime} \boldsymbol{\rho}_{t+1}} \\
& =\operatorname{diag}\left(\boldsymbol{\phi}_{t}\right) \times \frac{\mathbf{1}_{N}+\boldsymbol{x}^{\prime}\left(\boldsymbol{I}_{K}-\boldsymbol{M}\right)^{-1} \boldsymbol{e}_{t+1}}{1+\boldsymbol{\phi}_{t}^{\prime} \boldsymbol{\rho}_{t+1}} .
\end{aligned}
$$

We highlight that it is indeed possible to factor out the term $\operatorname{diag}\left(\boldsymbol{x} \phi_{t}\right)$ in the matrix inversion. Since $\lim _{q \rightarrow \infty} \boldsymbol{M}^{q}=\mathbf{0}_{K, K}$, the expression in the proposition is simply the explicit version of the above equation with the inverse matrix replaced by its Neumann series.

\section{Appendix H. Proof of Theorem 10}

In order to ease readability, we have divided the proof into three parts.

STEP ONE: Asymptotic behavior of $M$ when $\phi_{t, 1} \rightarrow 1$.

In the statement of the theorem, the first agent is the one with maximum $R_{n}$, that is $R_{1}>R_{j}$ for $j=2, \ldots, N$. We henceforth suppose that $\phi_{t, 1} \geq 1-\delta(\epsilon)$, which is equivalent to $\sum_{j=2}^{N} \phi_{t, j} \leq \delta(\epsilon)$. For improved notational clarity, we write $\boldsymbol{M}_{*}$ for $\boldsymbol{M}(1)$. The second point of Lemma 12 implies that the matrices $\boldsymbol{M}$ and $\boldsymbol{M}_{*}$ are close, thus it is useful to understand what happens when $\boldsymbol{M}$ is replaced by $\boldsymbol{M}_{*}$ in the numerator of (G.1):

$$
\begin{aligned}
\mathbf{1}_{N}+\boldsymbol{\rho}_{*} & =\mathbf{1}_{N}+\boldsymbol{x}^{\prime}\left(\boldsymbol{I}_{K}-\boldsymbol{M}_{*}\right)^{-1} \overline{\boldsymbol{e}} \\
& =\mathbf{1}_{N}+\boldsymbol{x}^{\prime}\left(\boldsymbol{I}_{K}-\mathbf{1}_{K} \boldsymbol{x}_{1}^{\prime}\right)^{-1} \overline{\boldsymbol{e}} \\
& =\mathbf{1}_{N}+\boldsymbol{x}^{\prime}\left(\boldsymbol{I}_{K}+\frac{\mathbf{1}_{K} \boldsymbol{x}_{1}^{\prime}}{1-\lambda_{1}}\right) \overline{\boldsymbol{e}}
\end{aligned}
$$

where in the third line we have used the Sherman-Morrison identity.

STEP TWO: the crucial decomposition

Now, let us consider an agent $n \in\{2, \ldots, N\}$ and compare the ratios $\phi_{t+1, j} / \phi_{t, j}$ between agent one and agent $n$. From (37), we deduce

$$
\begin{aligned}
\frac{\phi_{t+1,1}}{\phi_{t, 1}}-\frac{\phi_{t+1, n}}{\phi_{t, n}} & =c\left(\sum_{j=1}^{K} \sum_{l=1}^{K}\left(x_{1}^{(j)}-x_{n}^{(j)}\right) \sum_{q=0}^{\infty}\left[\boldsymbol{M}^{q}\right]_{j l} e^{(l)}\right) \\
& =c\left(\sum_{j=1}^{K} \sum_{l=1}^{K}\left(x_{1}^{(j)}-x_{n}^{(j)}\right) \sum_{q=0}^{\infty}\left\{\left[\boldsymbol{M}^{q}\right]_{j l}-\left[\boldsymbol{M}_{*}^{q}\right]_{j l}+\left[\boldsymbol{M}_{*}^{q}\right]_{j l}\right\} e^{(l)}\right)
\end{aligned}
$$

where $c=\left(1+\phi_{t}^{\prime} \boldsymbol{\rho}_{t+1}\right)^{-1}>0$ is bounded. We split this expression into two and from point iii) in Lemma 12 , it is clear that the first part

$$
c\left(\sum_{j=1}^{K} \sum_{l=1}^{K}\left(x_{1}^{(j)}-x_{n}^{(j)}\right) \sum_{q=0}^{\infty}\left\{\left[\boldsymbol{M}^{q}\right]_{j l}-\left[\boldsymbol{M}_{*}^{q}\right]_{j l}\right\} e^{(l)}\right)
$$


can be shrunk as much as desired so that when $\delta(\epsilon)$ is sufficiently small, the spread in variation of market shares is driven by the second (constant) part:

$$
\begin{aligned}
v_{1 n} & =c\left(\sum_{j=1}^{K} \sum_{l=1}^{K}\left(x_{1}^{(j)}-x_{n}^{(j)}\right) \sum_{q=0}^{\infty}\left[\boldsymbol{M}_{*}^{q}\right]_{j l} e^{(l)}\right) \\
& =c\left(\left(\boldsymbol{x}_{1}^{\prime}-\boldsymbol{x}_{n}^{\prime}\right)\left(\boldsymbol{I}_{K}+\frac{\mathbf{1}_{K} \boldsymbol{x}_{1}^{\prime}}{1-\lambda_{1}}\right) \overline{\boldsymbol{e}}\right) \\
& =c\left([\boldsymbol{x} \overline{\boldsymbol{e}}]_{1}-[\boldsymbol{x} \overline{\boldsymbol{e}}]_{n}+\frac{\lambda_{1}}{1-\lambda_{1}}[\boldsymbol{x} \overline{\boldsymbol{e}}]_{1}-\frac{\lambda_{n}}{1-\lambda_{1}}[\boldsymbol{x} \overline{\boldsymbol{e}}]_{1}\right) \\
& =\frac{c}{1-\lambda_{1}}\left(\left[\boldsymbol{x}^{\prime} \overline{\boldsymbol{e}}\right]_{1}\left(1-\lambda_{n}\right)-\left[\boldsymbol{x}^{\prime} \overline{\boldsymbol{e}}\right]_{n}\left(1-\lambda_{1}\right)\right) \\
& =c\left(1-\lambda_{n}\right)\left(R_{1}-R_{n}\right)>0,
\end{aligned}
$$

where we have used expression (H.1) in the second equality. The strict inequality stems directly from $R_{1}>R_{n}$.

In sum, the difference between the relative variation in shares consists of $v_{1 n}>0$ plus a term that can be made arbitrarily small through $\delta(\epsilon)$. Thus, for $\delta(\epsilon)$ sufficiently small, $\phi_{t, 1} \geq 1-\delta(\epsilon)$ will in turn imply $\phi_{t+s, 1} \geq 1-\epsilon$ for all future dates $t+s$.

\section{STEP THREE: the other market shares}

Step 2 proved the stability of the fixed point with $\phi_{t, 1}=1$ when $R_{1}>R_{j}$ for $j=2, \ldots, N$. In Step 3 we consider the stability of the fixed point with $\phi_{t, n} \rightarrow 1$ for an arbitrary $n \neq 1$ where we still have $R_{1}>R_{n}$ for $n=2, \ldots, N$. In particular, it still holds that $R_{1}>R_{n}$. The derivation of $\phi_{t+1,1} / \phi_{t, 1}-\phi_{t+1, n} / \phi_{t, n}>0$ is essentially the same as previously. Therefore, only the new expression of $v_{1 n}$ is detailed below.

From what we have shown previously, it appears clearly that for a sufficiently small $\delta(\epsilon)$, equation (H.2) is dominated by the term $v_{1 n}$ in (H.4). If we change the assumption over $\phi$ to an arbitrary $n$ (i.e., $\phi_{t, n} \geq 1-\delta(\epsilon)$ ), then $\boldsymbol{M}_{*}=\boldsymbol{M}(n)$ and the term reads

$$
\begin{aligned}
v_{1 n} & =c\left(\left(\boldsymbol{x}_{1}^{\prime}-\boldsymbol{x}_{n}^{\prime}\right)\left(\boldsymbol{I}_{K}+\frac{\mathbf{1}_{K} \boldsymbol{x}_{n}^{\prime}}{1-\lambda_{n}}\right) \overline{\boldsymbol{e}}\right) \\
& =\frac{c}{1-\lambda_{n}}\left(\left[\boldsymbol{x}^{\prime} \overline{\boldsymbol{e}}\right]_{1}\left(1-\lambda_{n}\right)-\left[\boldsymbol{x}^{\prime} \overline{\boldsymbol{e}}\right]_{n}\left(1-\lambda_{1}\right)\right) \\
& >0 .
\end{aligned}
$$

Compared to the original formula (H.4), it is only the multiplicative constant that has changed. Again, this means that in the new configuration in which another agent comes close to dominating the market $\left(\phi_{t, n} \geq 1-\delta(\epsilon)\right)$, the largest increase in market share will be that of the first agent. Thus, it is not possible that $\phi_{t, n}$ increases to one because $\phi_{t, 1}$ will never decrease to zero. In conclusion, 1 can only be a Lyapunov equilibrium for the agent with maximum characteristic ratio.

\section{Appendix I. Proof of Proposition 11}

We summarize the principle underlying the proof of Theorem 10 as follows: when $\phi_{t, 1}$ is sufficiently close to 1 , then

$$
\frac{\phi_{t+1,1}}{\phi_{t, 1}}-\frac{\phi_{t+1, n}}{\phi_{t, n}}=\epsilon+c\left(1-\lambda_{n}\right)\left(R_{1}-R_{n}\right), \quad n \neq 1
$$


where $\epsilon$ is arbitrarily small. The idea behind the generalization to random dividends is to exploit the (possibly small) space between $R_{1}$ and $R_{n}$ in the above expression. When the dividends are random, it translates into

$$
\frac{\phi_{t+1,1}}{\phi_{t, 1}}-\frac{\phi_{t+1, n}}{\phi_{t, n}}=\epsilon\left(\boldsymbol{e}_{t+1}\right)+c\left(1-\lambda_{n}\right)\left(\frac{\left[\boldsymbol{x}^{\prime} \boldsymbol{e}_{t+1}\right]_{1}}{1-\lambda_{1}}-\frac{\left[\boldsymbol{x}^{\prime} \boldsymbol{e}_{t+1}\right]_{n}}{1-\lambda_{n}}\right), \quad n \neq 1,
$$

and given the form (H.3), $\epsilon\left(\boldsymbol{e}_{t+1}\right)$ can be bounded by a multiple of $\epsilon$ (average dividends can be replaced by the upper bound of their supports to obtain an upper bound). Hence the relative variation of the first market share is again driven by the second term and is the highest across all agents (and hence positive) when

$$
\frac{\sum_{j=1}^{K} x_{1}^{(j)} e_{t+1}^{(j)}}{1-\lambda_{1}}-\frac{\sum_{j=1}^{K} x_{n}^{(j)} e_{t+1}^{(j)}}{1-\lambda_{n}}>0, \quad n \neq 1 .
$$

Now, we recall that we already have that $R_{1}>R_{n}$, i.e.,

$$
\frac{\sum_{j=1}^{K} x_{1}^{(j)} \bar{e}^{(j)}}{1-\lambda_{1}}>\frac{\sum_{j=1}^{K} x_{n}^{(j)} \bar{e}^{(j)}}{1-\lambda_{n}}, \quad n \neq 1 .
$$

Let us define the perturbation away from the mean dividend $\xi_{t+1}^{(j)}=e_{t+1}^{(j)}-\bar{e}^{j}$. If it is bounded in absolute terms by $\kappa>0$, i.e., $\left|\xi_{t}^{(j)}\right| \leq \kappa$, then

$$
\begin{aligned}
\frac{\sum_{j=1}^{K} x_{1}^{(j)}\left(\bar{e}^{(j)}+\xi_{t+1}^{(j)}\right)}{1-\lambda_{1}}-\frac{\sum_{j=1}^{K} x_{n}^{(j)}\left(\bar{e}^{(j)}+\xi_{t+1}^{(j)}\right)}{1-\lambda_{n}} & \geq \frac{\sum_{j=1}^{K} x_{1}^{(j)}\left(\bar{e}^{(j)}-\kappa\right)}{1-\lambda_{1}}-\frac{\sum_{j=1}^{K} x_{n}^{(j)}\left(\bar{e}^{(j)}+\kappa\right)}{1-\lambda_{n}} \\
& =R_{1}-R_{n}-\kappa\left(\frac{\lambda_{1}}{1-\lambda_{1}}+\frac{\lambda_{n}}{1-\lambda_{n}}\right)
\end{aligned}
$$

so that (I.1) holds whenever

$$
\kappa<\frac{R_{1}-R_{n}}{\left(\frac{\lambda_{1}}{1-\lambda_{1}}+\frac{\lambda_{n}}{1-\lambda_{n}}\right)} .
$$

Thus, for a given vector of average dividends $\overline{\boldsymbol{e}}$, the Lyapunov stability of 1 for $\phi_{t, 1}$ is ensured as long as for all dates $t \geq 1$ (I.1) holds, which is guaranteed if the support of $e_{t}^{(k)}$ is inside the interval

$$
I_{k}=\left(\max \left(0, \bar{e}^{(k)}-\min _{n \neq 1} \frac{R_{1}-R_{n}}{\left(\frac{\lambda_{1}}{1-\lambda_{1}}+\frac{\lambda_{n}}{1-\lambda_{n}}\right)}\right), \bar{e}^{(k)}+\min _{n \neq 1} \frac{R_{1}-R_{n}}{\left(\frac{\lambda_{1}}{1-\lambda_{1}}+\frac{\lambda_{k}}{1-\lambda_{n}}\right)}\right) .
$$

\section{Appendix J. Diagonal demands}

In this section, we explore a special case in which the demand matrix $\boldsymbol{x}$ is diagonal (this implicitly assumes $N=K)$. We write $x_{n}^{(k)}=x_{n}$ if $n=k$ and $x_{n}^{(k)}=0$ otherwise. The crucial law of motion of market shares (32) then simplifies to

$$
\frac{\phi_{t+1, n}}{\phi_{t, n}} \propto 1+\frac{x_{n} e_{t+1}^{(n)}}{1-x_{n}}
$$

from which we conclude that in the deterministic case, the statement of Theorem (10) holds even if (6) is violated. Moreover, a surprising property of the model is that upon equilibrium, the returns satisfy $\boldsymbol{r}^{*}=\boldsymbol{R}$, i.e., price returns equal the characteristic ratios. This is inferred from the second line of identity (7).

This simple example illustrates the central role of characteristic ratios in the model and highlights that the survival condition of Theorem (10) can remain valid when condition (6) fails to hold asymptotically. 


\section{Appendix K. Evolution of market shares upon equilibrium}

Proposition 13. We assume condition (6) is fulfilled so that all demands are nonzero. In this case, when $\boldsymbol{r}_{t}=r^{*} \mathbf{1}_{K}$ (returns have reached their equilibrium values), agents with maximal characteristic ratio $R_{n}$ have increasing market shares. Moreover, the strategy that maximizes $R_{n}$ is the only one that has non-decreasing market shares independently of the level of other market shares.

The proof of the proposition requires an intermediate lemma.

Lemma 14. For $N$-dimensional positive vectors $\boldsymbol{x}, \boldsymbol{y}$ and $\boldsymbol{\phi}$, the following holds:

$$
\max _{i \in\{1, \ldots, N\}}\left(\frac{x_{i}}{y_{i}}\right) \geq \frac{\phi^{\prime} \boldsymbol{x}}{\boldsymbol{\phi}^{\prime} \boldsymbol{y}} .
$$

Proof. We proceed iteratively. For $N=1$, the statement is clearly true. For any $N \geq 1$, we assume that (K.1) holds and we define

$$
f\left(\phi_{N+1}\right)=\frac{\sum_{n=1}^{N} \phi_{n} x_{n}+\phi_{N+1} x_{N+1}}{\sum_{n=1}^{N} \phi_{n} y_{n}+\phi_{N+1} y_{N+1}}
$$

and

$$
\begin{aligned}
f^{\prime}\left(\phi_{N+1}\right) & =\frac{x_{N+1}\left(\sum_{n=1}^{N} \phi_{n} y_{n}+\phi_{N+1} y_{N+1}\right)-y_{N+1}\left(\sum_{n=1}^{N} \phi_{n} x_{n}+\phi_{N+1} x_{N+1}\right)}{\left(\sum_{n=1}^{N} \phi_{n} y_{n}+\phi_{N+1} y_{N+1}\right)^{2}} \\
& =\frac{x_{N+1} \sum_{n=1}^{N} \phi_{n} y_{n}-y_{N+1} \sum_{n=1}^{N} \phi_{n} x_{n}}{\left(\sum_{n=1}^{N} \phi_{n} y_{n}+\phi_{N+1} y_{N+1}\right)^{2}}
\end{aligned}
$$

so that $f$ is either increasing or decreasing, depending on whether

$$
\frac{x_{N+1}}{y_{N+1}} \geq \frac{\sum_{n=1}^{N} \phi_{n} x_{n}}{\sum_{n=1}^{N} \phi_{n} y_{n}}
$$

or not. This condition is independent of $\phi_{N+1}$. We provide a scheme of these two cases in Figure K.9. Accordingly, an upper bound for $f\left(\phi_{N+1}\right)$ is

- either $x_{N+1} / y_{N+1}$ (for $\phi_{N+1} \rightarrow+\infty$ when $f$ is increasing: point B in the right graph),

- or $\frac{\sum_{n=1}^{N} \phi_{n} x_{n}}{\sum_{n=1}^{N} \phi_{n} y_{n}}$ (for $\phi_{N+1}=0$ when $f$ is decreasing: point A in the left graph).

Given that (K.1) holds, this means that the upper bound is also true when one element is added to the set. Hence, the statement remains true and the result is proven iteratively.

We now turn to the proof of Proposition 13. Our goal is to show that the profit of agents with maximal $R_{n}$ is larger than the average share-weighted profit. Indeed, by equation (9), this implies an increase of market share. We recall that in this case all returns (in the cross-section) are equal to $r^{*}=\frac{\left(\phi^{*}\right)^{\prime}\left(\boldsymbol{x}^{*}\right)^{\prime} \overline{\boldsymbol{e}}}{1-\left(\boldsymbol{\phi}^{*}\right)^{\prime}\left(\boldsymbol{x}^{*}\right)^{\prime} \mathbf{1}_{K}}$.. The dynamic version of equation (C.1) when shares only are allowed to evolve as

$$
\phi_{t+1}=\operatorname{diag}\left(\phi_{t}\right) \frac{\mathbf{1}_{N}+\left(\boldsymbol{x}^{*}\right)^{\prime}\left(r^{*} \mathbf{1}_{K}+\overline{\boldsymbol{e}}\right)}{1+\left(\boldsymbol{\phi}_{t}\right)^{\prime}\left(\boldsymbol{x}^{*}\right)^{\prime}\left(r^{*} \mathbf{1}_{K}+\overline{\boldsymbol{e}}\right)}
$$

(equivalently, this is the simplified version of equation (9)). The relative evolution of shares is thus

$$
\operatorname{diag}\left(\boldsymbol{\phi}_{t}\right)^{-1} \boldsymbol{\phi}_{t+1}-\mathbf{1}_{N}=\frac{\left(\boldsymbol{x}^{*}\right)^{\prime}\left(r^{*} \mathbf{1}_{K}+\overline{\boldsymbol{e}}\right)-\left(\left(\boldsymbol{\phi}_{t}\right)^{\prime}\left(\boldsymbol{x}^{*}\right)^{\prime}\left(r^{*} \mathbf{1}_{K}+\overline{\boldsymbol{e}}\right)\right) \times \mathbf{1}_{N}}{1+\left(\boldsymbol{\phi}_{t}\right)^{\prime}\left(\boldsymbol{x}^{*}\right)^{\prime}\left(r^{*} \mathbf{1}_{K}+\overline{\boldsymbol{e}}\right)}
$$



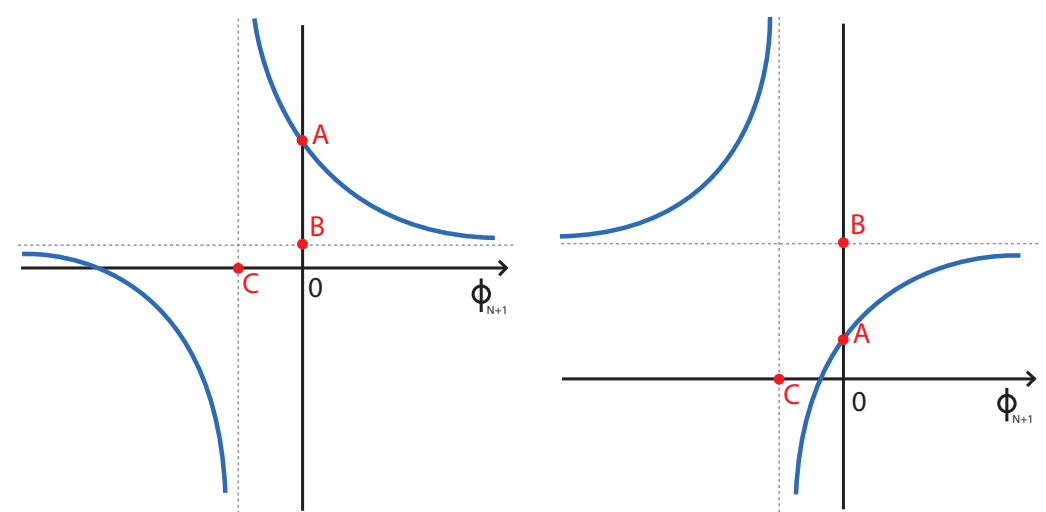

Figure K.9: Plot decreasing (left) and increasing (right) $f$ function defined in (K.2) in blue. The important points are A: $f(0)=\frac{\sum_{n=1}^{N} \phi_{n} x_{n}}{\sum_{n=1}^{N} \phi_{n} y_{n}} ; \mathbf{B}: f(+\infty)=x_{N+1} / y_{N+1}$ and the singularity location $\mathbf{C}: \phi_{N+1}=-\frac{\sum_{n=1}^{N} \phi_{n} y_{n}}{y_{N+1}}$.

Because the denominator is positive, agent $n$ 's share will increase when the numerator is positive, i.e., when

$$
\sum_{k=1}^{K} x_{n}^{(k)}\left(r^{*}+\bar{e}^{(k)}\right)-\sum_{i=1}^{N} \phi_{i} \sum_{k=1}^{K} x_{i}^{(k)}\left(r^{*}+\bar{e}^{(k)}\right) \geq 0
$$

$(1) \Leftrightarrow \quad r^{*} \geq \frac{\sum_{k=1}^{K} \sum_{i=1}^{N} \phi_{i}\left(x_{i}^{(k)}-x_{n}^{(k)}\right) \bar{e}^{(k)}}{\sum_{k=1}^{K} \sum_{i=1}^{N} \phi_{i}\left(x_{n}^{(k)}-x_{i}^{(k)}\right)} \quad$ (indications are provided below)

$(2) \Leftrightarrow \frac{\sum_{k=1}^{K} \sum_{i=1}^{N} \phi_{i} x_{i}^{(k)} \bar{e}^{(k)}}{1-\sum_{k=1}^{K} \sum_{i=1}^{N} \phi_{i} x_{i}^{(k)}} \geq \frac{\sum_{k=1}^{K} \sum_{i=1}^{N} \phi_{i}\left(x_{i}^{(k)}-x_{n}^{(k)}\right) \bar{e}^{(k)}}{\sum_{k=1}^{K} \sum_{i=1}^{N} \phi_{i}\left(x_{n}^{(k)}-x_{i}^{(k)}\right)}$

$(3) \Leftrightarrow\left(\sum_{k=1}^{K} \sum_{i=1}^{N} \phi_{i} x_{i}^{(k)} \bar{e}^{(k)}\right)\left(\sum_{k=1}^{K} \sum_{i=1}^{N} \phi_{i}\left(x_{n}^{(k)}-x_{i}^{(k)}\right)\right) \geq\left(\sum_{k=1}^{K} \sum_{i=1}^{N} \phi_{i}\left(x_{i}^{(k)}-x_{n}^{(k)}\right) \bar{e}^{(k)}\right)\left(\sum_{k=1}^{K} \sum_{i=1}^{N} \phi_{i}\left(K^{-1}-x_{i}^{(k)}\right)\right)$

$(4) \Leftrightarrow\left(\sum_{k=1}^{K} \sum_{i=1}^{N} \phi_{i} x_{i}^{(k)} \bar{e}^{(k)}\right)\left(\sum_{k=1}^{K} \sum_{i=1}^{N} \phi_{i} x_{n}^{(k)}\right) \geq-\left(\sum_{k=1}^{K} \sum_{i=1}^{N} \phi_{i} x_{n}^{(k)} \bar{e}^{(k)}\right)\left(\sum_{k=1}^{K} \sum_{i=1}^{N} \phi_{i}\left(K^{-1}-x_{i}^{(k)}\right)\right)$

$$
+\left(\sum_{k=1}^{K} \sum_{i=1}^{N} \phi_{i} x_{i}^{(k)} \bar{e}^{(k)}\right)\left(\sum_{k=1}^{K} \sum_{i=1}^{N} \phi_{i} K^{-1}\right)
$$

$(5) \Leftrightarrow\left(\sum_{k=1}^{K} \sum_{i=1}^{N} \phi_{i} x_{i}^{(k)} \bar{e}^{(k)}\right)\left(\sum_{k=1}^{K} \sum_{i=1}^{N} \phi_{i}\left(x_{n}^{(k)}-K^{-1}\right)\right) \geq-\left(\sum_{k=1}^{K} \sum_{i=1}^{N} \phi_{i} x_{n}^{(k)} \bar{e}^{(k)}\right)\left(\sum_{k=1}^{K} \sum_{i=1}^{N} \phi_{i}\left(K^{-1}-x_{i}^{(k)}\right)\right)$

(6) $\Leftrightarrow \frac{\sum_{k=1}^{K} \sum_{i=1}^{N} \phi_{i} x_{n}^{(k)} \bar{e}^{(k)}}{\sum_{k=1}^{K} \sum_{i=1}^{N} \phi_{i}\left(K^{-1}-x_{n}^{(k)}\right)} \geq \frac{\sum_{k=1}^{K} \sum_{i=1}^{N} \phi_{i} x_{i}^{(k)} \bar{e}^{(k)}}{\sum_{k=1}^{K} \sum_{i=1}^{N} \phi_{i}\left(K^{-1}-x_{i}^{(k)}\right)}$

$(7) \Leftrightarrow \frac{\sum_{k=1}^{K} x_{n}^{(k)} \bar{e}^{(k)}}{1-\sum_{k=1}^{K} x_{n}^{(k)}} \geq \frac{\sum_{k=1}^{K} \sum_{i=1}^{N} \phi_{i} x_{i}^{(k)} \bar{e}^{(k)}}{1-\sum_{k=1}^{K} \sum_{i=1}^{N} \phi_{i} x_{i}^{(k)}}$,

where we have used the property $\sum_{i=1}^{N} \phi_{i}=1$ several times and omitted the star superscript over $x$ and time-dependence of the $\phi_{i}$ for notational simplicity. (1) is obvious; (2) comes from the expression of $r^{*}$; (3) is obvious; in (4) we simply deleted one common term on both sides; (5) is a rearrangement (putting the term below in (4) on the l.h.s.); (6) and (7) are straightforward.

Hence, by Lemma 14, upon equilibrium, agents with maximal $R_{n}$ do not have decreasing market shares. Moreover, this result is independent of $\phi$. Indeed, (7) may hold for other agents, but it will 
depend on the configuration of market shares.

\section{Appendix L. Replacing the safe asset by consumption}

In this section, the time-dependence of variables will always be indicated, even when dealing with equilibrium values. This allows to maintain generality in all expressions along the way of the reasoning. Throughout the section, it will most often be assumed that aggregate demands are non-null and that both individual demands and dividends are constant.

One of the defining equations of the law of motion in our system is Equation 1:

$$
W_{t+1, n}\left(\boldsymbol{P}_{t+1}\right)=\left(1-\sum_{k=1}^{K} x_{t, n}^{(k)}\right) W_{t, n}+W_{t, n} \sum_{k=1}^{K} x_{t, n}^{(k)} \frac{P_{t+1}^{(k)}+D_{t+1}^{(k)}}{P_{t}^{(k)}}
$$

in which it is clear that the first term on the r.h.s. pertains to the money invested in the riskless asset. In the body of the paper, we get rid of $r_{f}$ by considering discounted quantities, hence we have set $r_{f}=0$ to ease the presentation of the reasoning. In Evstigneev et al. (2008), the agent consumes a portion $1-\rho$ of his wealth, hence the dynamics take the form

$$
\boldsymbol{w}_{t+1}=\rho \operatorname{diag}\left(\boldsymbol{w}_{t}\right) \boldsymbol{x}_{t}^{\prime}\left(\operatorname{diag}\left(\boldsymbol{p}_{t}\right)^{-1} \boldsymbol{p}_{t+1}+\boldsymbol{e}_{t+1}\right),
$$

which is the vector equivalent of (39). In this case, with the notations of the paper, plain algebra leads to

$$
\boldsymbol{p}_{t+1}=\rho \operatorname{diag}\left(\boldsymbol{p}_{t}\right)\left(\operatorname{diag}\left(\boldsymbol{x}_{t} \boldsymbol{w}_{t}\right)-\rho \boldsymbol{x}_{t+1} \operatorname{diag}\left(\boldsymbol{w}_{t}\right) \boldsymbol{x}_{t}^{\prime}\right)^{-1}\left(\boldsymbol{x}_{t+1} \operatorname{diag}\left(\boldsymbol{w}_{t}\right) \boldsymbol{x}_{t}^{\prime} \boldsymbol{e}_{t+1}\right),
$$

and hence to

$$
\begin{aligned}
\boldsymbol{r}_{t+1} & =\left(\operatorname{diag}\left(\boldsymbol{x}_{t} \boldsymbol{w}_{t}\right)-\rho \boldsymbol{x}_{t+1} \operatorname{diag}\left(\boldsymbol{w}_{t}\right) \boldsymbol{x}_{t}^{\prime}\right)^{-1}\left(\boldsymbol{x}_{t+1} \operatorname{diag}\left(\boldsymbol{w}_{t}\right) \boldsymbol{x}_{t}^{\prime}\left(\rho \boldsymbol{e}_{t+1}+\mathbf{1}_{K}\right)-\boldsymbol{x}_{t} \boldsymbol{w}_{t}\right), \\
& =\left(\operatorname{diag}\left(\boldsymbol{x}_{t} \boldsymbol{\phi}_{t}\right)-\rho \boldsymbol{x}_{t+1} \operatorname{diag}\left(\boldsymbol{\phi}_{t}\right) \boldsymbol{x}_{t}^{\prime}\right)^{-1}\left(\boldsymbol{x}_{t+1} \operatorname{diag}\left(\boldsymbol{\phi}_{t}\right) \boldsymbol{x}_{t}^{\prime}\left(\rho \boldsymbol{e}_{t+1}+\mathbf{1}_{K}\right)-\boldsymbol{x}_{t} \boldsymbol{\phi}_{t}\right),
\end{aligned}
$$

where in the second line we have simply replaced wealths by market shares. From (L.1), we infer the dynamics of market shares:

$$
\phi_{t+1}=\frac{\operatorname{diag}\left(\phi_{t}\right)\left(\boldsymbol{x}_{t}^{\prime}\left(\boldsymbol{r}_{t+1}+\boldsymbol{e}_{t+1}\right)\right)}{\boldsymbol{\phi}_{t}^{\prime} \boldsymbol{x}_{t}^{\prime}\left(\boldsymbol{r}_{t+1}+\boldsymbol{e}_{t+1}\right)} .
$$

Since $\rho$ is equal across agents, it disappears in the above line.

At a $\operatorname{PCE}\left(\phi_{t+\mathbf{1}}=\phi_{t}\right.$ and $\left.\boldsymbol{x}_{t+1}=\boldsymbol{x}_{t}\right)$, and assuming that all agents survive and that aggregate demands are non null, the deterministic skeleton must satisfy $\boldsymbol{x}_{t}^{\prime}\left(\boldsymbol{r}_{t+1}+\boldsymbol{e}_{t+1}\right)=c \mathbf{1}_{N}$, where $c=$ $\boldsymbol{\phi}_{t}^{\prime} \boldsymbol{x}_{t}^{\prime}\left(\boldsymbol{r}_{t+1}+\boldsymbol{e}_{t+1}\right)$. As expected, the demand-weighted total returns are equal across agents, as in Appendix C. From (L.2), we have

$$
\left(\operatorname{diag}\left(\boldsymbol{x}_{t} \boldsymbol{\phi}_{t}\right)-\rho \boldsymbol{x}_{t+1} \operatorname{diag}\left(\boldsymbol{\phi}_{t}\right) \boldsymbol{x}_{t}^{\prime}\right) \boldsymbol{r}_{t+1}=\boldsymbol{x}_{t+1} \operatorname{diag}\left(\boldsymbol{\phi}_{t}\right) \boldsymbol{x}_{t}^{\prime}\left(\rho \boldsymbol{e}_{t+1}+\mathbf{1}_{K}\right)-\boldsymbol{x}_{t} \boldsymbol{\phi}_{t},
$$

that is,

$$
\rho \boldsymbol{x}_{t+1} \operatorname{diag}\left(\boldsymbol{\phi}_{t}\right) \boldsymbol{x}_{t}^{\prime}\left(\boldsymbol{r}_{t+1}+\boldsymbol{e}_{t+1}\right)=\operatorname{diag}\left(\boldsymbol{x}_{t} \boldsymbol{\phi}_{t}\right) \boldsymbol{r}_{t+1}+\boldsymbol{x}_{t} \boldsymbol{\phi}_{t}-\boldsymbol{x}_{t+1} \operatorname{diag}\left(\boldsymbol{\phi}_{t}\right) \boldsymbol{x}_{t}^{\prime} \mathbf{1}_{K} .
$$

Plugging $\boldsymbol{x}_{t}^{\prime}\left(\boldsymbol{r}_{t+1}+\boldsymbol{e}_{t+1}\right)=c \mathbf{1}_{N}$ gives (with $\boldsymbol{x}_{t+1}=\boldsymbol{x}_{t}$ and nonzero aggregate demands)

$$
\boldsymbol{r}_{t+1}=(c \rho-1) \mathbf{1}_{K}+\operatorname{diag}\left(\boldsymbol{x}_{t} \boldsymbol{\phi}_{t}\right)^{-1} \boldsymbol{x}_{t+1} \operatorname{diag}\left(\boldsymbol{\phi}_{t}\right) \boldsymbol{x}_{t}^{\prime} \mathbf{1}_{K} .
$$


If we assume $\boldsymbol{x}_{t}^{\prime} \mathbf{1}_{K}=\mathbf{1}_{N}$ and constant investment shares, this simplifies to $\boldsymbol{r}_{t+1}=c \rho \mathbf{1}_{K}$, which is very similar to the first part of Proposition 4 in which equilibrium returns are all equal.

We pursue the layout by adding and subtracting $\operatorname{diag}\left(\boldsymbol{x}_{t} \boldsymbol{\phi}_{t}\right) \boldsymbol{e}_{t+1}$ to the r.h.s. of (L.4) to get

$$
\begin{aligned}
\boldsymbol{r}_{t+1}+\boldsymbol{e}_{t+1} & =\left(\operatorname{diag}\left(\boldsymbol{x}_{t} \boldsymbol{\phi}_{t}\right)-\rho \boldsymbol{x}_{t+1} \operatorname{diag}\left(\boldsymbol{\phi}_{t}\right) \boldsymbol{x}_{t}^{\prime}\right)^{-1}\left(\boldsymbol{x}_{t+1} \operatorname{diag}\left(\boldsymbol{\phi}_{t}\right) \boldsymbol{x}_{t}^{\prime} \mathbf{1}_{K}-\boldsymbol{x}_{t} \boldsymbol{\phi}_{t}+\operatorname{diag}\left(\boldsymbol{x}_{t} \boldsymbol{\phi}_{t}\right) \boldsymbol{e}_{t+1}\right) \\
& =\left(\boldsymbol{I}_{K}-\rho \boldsymbol{M}\right)^{-1}\left(\boldsymbol{M} \mathbf{1}_{K}-\mathbf{1}_{K}+\boldsymbol{e}_{t+1}\right),
\end{aligned}
$$

where the second line holds for fixed demands $\boldsymbol{x}_{t}=\boldsymbol{x}$ and $\boldsymbol{M}$ is defined in (36). This is the equation that will drive the dynamics of market shares, via (L.3). If we equate the first line of the above equation with $\boldsymbol{e}_{t+1}$ added to (L.5) and if we assume $K \geq N$ so that we can discard $\boldsymbol{x}_{t}$, then it can be shown that upon a PCE,

$$
\operatorname{diag}\left(\boldsymbol{\phi}_{t}\right)\left(c \mathbf{1}_{N}-(c \rho-1) \boldsymbol{x}_{t}^{\prime} \mathbf{1}_{K}-\boldsymbol{x}_{t}^{\prime} \operatorname{diag}\left(\boldsymbol{x}_{t} \boldsymbol{\phi}_{t}\right)^{-1} \boldsymbol{x}_{t} \operatorname{diag}\left(\boldsymbol{\phi}_{t}\right) \boldsymbol{x}_{t}^{\prime} \mathbf{1}_{K}-\boldsymbol{x}_{t}^{\prime} \boldsymbol{e}_{t+1}\right)=\mathbf{0}_{N},
$$

so that if all agents survive,

$$
\tilde{\boldsymbol{R}}=\left(\mathbf{1}_{N}-\rho \boldsymbol{x}_{t}^{\prime} \mathbf{1}_{K}\right)^{-1}\left(\boldsymbol{x}_{t}^{\prime} \boldsymbol{e}_{t+1}-\left(\boldsymbol{I}_{N}-\boldsymbol{x}_{t}^{\prime} \operatorname{diag}\left(\boldsymbol{x}_{t} \phi_{t}\right)^{-1} \boldsymbol{x}_{t} \operatorname{diag}\left(\phi_{t}\right)\right) \boldsymbol{x}_{t}^{\prime} \mathbf{1}_{K}\right) \propto \mathbf{1}_{N} .
$$

This ratio is the counterpart of $\boldsymbol{R}$ is the body of the paper. Unfortunately, the additional complicated term is $\phi_{t}$-dependent. Thus, it is impossible to infer a simple condition of survival a priori from this expression in the general case. Nevertheless, if we impose $\boldsymbol{x}_{t}^{\prime} \mathbf{1}_{K}=\mathbf{1}_{N}$, then again strong simplifications occur and

$$
\tilde{\boldsymbol{R}}=\frac{\boldsymbol{x}_{t}^{\prime} \boldsymbol{e}_{t+1}}{1-\rho} .
$$

This is, up to a multiplicative constant, the same value as in the body of the paper (under uniform leverages $\boldsymbol{x}_{t}^{\prime} \mathbf{1}_{K}=\lambda \mathbf{1}_{N}$ with $\lambda \in(0,1)$ : in the two models $\rho$ and $\lambda$ have a similar role).

Finally, in the spirit of the proof of Theorem 10, we unveil the asymptotic behavior of (L.6) when one (say, the first) agent dominates the market. In this case, $\boldsymbol{M} \rightarrow \mathbf{1}_{K} \boldsymbol{x}_{1}^{\prime}$ and the ShermanMorrison formula applied to (L.6) implies

$$
\boldsymbol{r}_{t+1}+\boldsymbol{e}_{t+1}=\left(\boldsymbol{I}_{K}+\frac{\rho}{1-\lambda_{1} \rho} \mathbf{1}_{K} \boldsymbol{x}_{1}^{\prime}\right)\left(\left(\lambda_{1}-1\right) \mathbf{1}_{K}+\boldsymbol{e}_{t+1}\right) .
$$

The difference in market share evolution between agent 1 and agent $n$ is likely to be asymptotically driven by the constant term

$$
\begin{aligned}
v_{1 n} & =\left(\boldsymbol{x}_{1}^{\prime}-\boldsymbol{x}_{\boldsymbol{n}}^{\prime}\right)\left(\boldsymbol{I}_{K}+\frac{\rho}{1-\lambda_{1} \rho} \mathbf{1}_{K} \boldsymbol{x}_{1}^{\prime}\right)\left(\left(\lambda_{1}-1\right) \mathbf{1}_{K}+\boldsymbol{e}_{t+1}\right) \\
& =\frac{1}{1-\lambda_{1} \rho}\left(\lambda_{1}^{2}-\lambda_{1}-\lambda_{1} \lambda_{n}+\lambda_{n}+\left\{\left(1-\rho \lambda_{n}\right)[\boldsymbol{x} \boldsymbol{e}]_{1}-\left(1-\rho \lambda_{1}\right)[\boldsymbol{x} \boldsymbol{e}]_{n}\right\}\right) .
\end{aligned}
$$

If the demand configuration is such that $\boldsymbol{x}^{\prime} \mathbf{1}_{K}=\boldsymbol{\lambda}=\lambda \mathbf{1}_{N}$, then $v_{1 n}>0 \Leftrightarrow[\boldsymbol{x e}]_{1}>[\boldsymbol{x e}]_{n}$, which is the same condition as in the baseline model. Likewise, it can be shown that if another agent sees his share increasing to one $\boldsymbol{M} \rightarrow \mathbf{1}_{K} \boldsymbol{x}_{n}^{\prime}$ for $n \neq 1$, then agent 1 will at some point see its market share increase faster than agent $n$. 


\section{References}

Algoet, P., Cover, T., 1988. Asymptotic optimality and asymptotic equipartition properties of log-optimum investment. Annals of Probability 16 (2), 876-898.

Amir, R., Evstigneev, I. V., Hens, T., Schenk-Hoppé, K. R., 2005. Market selection and survival of investment strategies. Journal of Mathematical Economics 41 (1), 105-122.

Amir, R., Evstigneev, I. V., Schenk-Hoppé, K. R., 2013. Asset market games of survival: a synthesis of evolutionary and dynamic games. Annals of Finance 9 (2), 121-144.

Anufriev, M., Bottazzi, G., 2010. Market equilibria under procedural rationality. Journal of Mathematical Economics 46 (6), 1140-1172.

Anufriev, M., Bottazzi, G., Marsili, M., Pin, P., 2012. Excess covariance and dynamic instability in a multi-asset model. Journal of Economic Dynamics and Control 36 (8), 1142-1161.

Anufriev, M., Bottazzi, G., Pancotto, F., 2006. Equilibria, stability and asymptotic dominance in a speculative market with heterogeneous traders. Journal of Economic Dynamics and Control 30 (9), 1787-1835.

Anufriev, M., Dindo, P., 2010. Wealth-driven selection in a financial market with heterogeneous agents. Journal of Economic Behavior \& Organization 73 (3), 327-358.

Barberis, N., Xiong, W., 2012. Realization utility. Journal of Financial Economics 104 (2), 251-271.

Benaïm, M., Weibull, J. W., 2003. Deterministic approximation of stochastic evolution in games. Econometrica 71 (3), 873-903.

Berman, A., Plemmons, R. J., 1994. Nonnegative Matrices in the Mathematical Sciences. Vol. 9. SIAM.

Besbes, O., Zeevi, A., 2012. Blind network revenue management. Operations Research 60 (6), $1537-1550$.

Bhamra, H. S., Uppal, R., 2014. Asset prices with heterogeneity in preferences and beliefs. Review of Financial Studies 27 (2), 519-580.

Blume, L., Easley, D., 1992. Evolution and market behavior. Journal of Economic Theory 58 (1), $9-40$.

Bottazzi, G., Dindo, P., 2014. Evolution and market behavior with endogenous investment rules. Journal of Economic Dynamics and Control 48, 121-146.

Breiman, L., 1960. Investment policies for expanding businesses optimal in a long-run sense. Naval Research Logistics Quarterly 7 (4), 647-651.

Brock, W. A., Hommes, C. H., 1998. Heterogeneous beliefs and routes to chaos in a simple asset pricing model. Journal of Economic Dynamics and Control 22 (8), 1235-1274.

Brock, W. A., Hommes, C. H., Wagener, F. O., 2005. Evolutionary dynamics in markets with many trader types. Journal of Mathematical Economics 41 (1), 7-42.

Buraschi, A., Trojani, F., Vedolin, A., 2014. When uncertainty blows in the orchard: Comovement and equilibrium volatility risk premia. Journal of Finance 69 (1), 101-137. 
Chiarella, C., Dieci, R., He, X.-Z., 2007. Heterogeneous expectations and speculative behavior in a dynamic multi-asset framework. Journal of Economic Behavior \& Organization 62 (3), 408-427.

Chiarella, C., Dieci, R., He, X.-Z., 2010. A framework for CAPM with heterogeneous beliefs. In: Nonlinear Dynamics in Economics, Finance and Social Sciences. Springer, pp. 353-369.

Chiarella, C., Dieci, R., He, X.-Z., Li, K., 2013. An evolutionary CAPM under heterogeneous beliefs. Annals of Finance 9 (2), 185-215.

Coqueret, G., 2017. Empirical properties of a heterogeneous agent model in large dimensions. Journal of Economic Dynamics and Control 77, 180-201.

Dieci, R., Foroni, I., Gardini, L., He, X.-Z., 2006. Market mood, adaptive beliefs and asset price dynamics. Chaos, Solitons and Fractals 29, 520-534.

Evstigneev, I. V., Hens, T., Schenk-Hoppé, K. R., 2008. Globally evolutionarily stable portfolio rules. Journal of Economic Theory 140 (1), 197-228.

Evstigneev, I. V., Hens, T., Schenk-Hoppe, K. R., 2009. Evolutionary finance. In: Handbook of financial markets: dynamics and evolution. North-Holland, pp. 507-566.

Finkelstein, M., Whitley, R., 1981. Optimal strategies for repeated games. Advances in Applied Probability 13 (2), 415-428.

Freidlin, M., Wentzell, A., 2012. Random perturbations of dynamical systems, 3rd Edition. Vol. 260 of Grundlehren der mathematischen Wissenschaften. Springer.

Frydman, C., Barberis, N., Camerer, C., Bossaerts, P., Rangel, A., 2014. Using neural data to test a theory of investor behavior: An application to realization utility. Journal of Finance 69 (2), 907-946.

Greene, W. H., 2000. Econometric analysis (Seventh edition). Prentice Hall.

Hens, T., Schenk-Hoppé, K. R., 2005. Evolutionary stability of portfolio rules in incomplete markets. Journal of Mathematical Economics 41 (1), 43-66.

Hommes, C., Huang, H., Wang, D., 2005. A robust rational route to randomness in a simple asset pricing model. Journal of Economic Dynamics and Control 29 (6), 1043-1072.

Ingersoll, J. E., Jin, L. J., 2012. Realization utility with reference-dependent preferences. Review of Financial Studies 26 (3), 723-767.

Kelly, J., 1956. A new interpretation of information rate. Bell Sys. Tech. Journal 35, 917-926.

Kogan, L., Ross, S., Wang, J., Westerfield, M. M., 2016. Market selection. Journal of Economic Theory 168, 209-236.

Koijen, R. S., Yogo, M., 2017. An equilibrium model of institutional demand and asset prices. SSRN Working Paper.

Leary, M. T., Michaely, R., 2011. Determinants of dividend smoothing: Empirical evidence. Review of Financial Studies 24 (10), 3197-3249.

Li, M., Zheng, H., Chong, T. T. L., Zhang, Y., 2016. The stock-bond comovements and crossmarket trading. Journal of Economic Dynamics and Control 73, 417-438. 
Lintner, J., 1956. Distribution of incomes of corporations among dividends, retained earnings, and taxes. American Economic Review 46 (2), 97-113.

MacLean, L. C., Thorp, E. O., Ziemba, W. T., 2011. The Kelly capital growth investment criterion: Theory and practice. Vol. 3. World Scientific.

Magnus, J. R., Neudecker, H., 2007. Matrix Differential Calculus. John Wiley and Sons.

McQueen, G., Shields, K., Thorley, S. R., 1997. Does the "Dow-10 investment strategy" beat the dow statistically and economically? Financial Analysts Journal, 66-72.

Medio, A., Lines, M., 2001. Nonlinear Dynamics. Cambridge University Press.

Meyer, C. D., 2000. Matrix Analysis and Applied Linear Algebra. SIAM.

Palczewski, J., Schenk-Hoppé, K. R., 2010. Market selection of constant proportions investment strategies in continuous time. Journal of Mathematical Economics 46 (2), 248-266.

Pantaleo, E., Tumminello, M., Lillo, F., Mantegna, R. N., 2011. When do improved covariance matrix estimators enhance portfolio optimization? An empirical comparative study of nine estimators. Quantitative Finance 11 (7), 1067-1080.

Sandroni, A., 2000. Do markets favor agents able to make accurate predictions? Econometrica $68(6), 1303-1341$.

Shapiro, D., 2009. Evolution of heterogeneous beliefs and asset overvaluation. Journal of Mathematical Economics 45 (3), 277-292.

Turtle, H., Wang, K., 2016. The benefits of improved covariance estimation. Journal of Empirical Finance 37, 233-246.

Visscher, S., Filbeck, G., 2003. Dividend-yield strategies in the canadian stock market. Financial Analysts Journal, 99-106.

Yan, H., 2008. Natural selection in financial markets: Does it work? Management Science 54 (11), 1935-1950.

Zhu, M., Wang, D., Guo, M., 2011a. Existence and stability of stationary solutions of nonlinear difference equations under random perturbations. Journal of Difference Equations and Applications $17(4), 587-602$.

Zhu, M., Wang, D., Guo, M., 2011b. Stochastic equilibria of an asset pricing model with heterogeneous beliefs and random dividends. Journal of Economic Dynamics and Control 35 (1), $131-147$. 